MURILLO FRAGUAS FRANCO NETO

\title{
TÉCNICA PARA O PROJETO DE UM AMPLIFICADOR OPERACIONAL FOLDED CASCODE, CLASSE AB, EM TECNOLOGIA CMOS.
}

Dissertação apresentada à Escola

Politécnica da Universidade de São

Paulo para obtenção do título de Mestre em Engenharia. 
MURILLO FRAGUAS FRANCO NETO

\section{TÉCNICA PARA O PROJETO DE UM AMPLIFICADOR OPERACIONAL FOLDED CASCODE, CLASSE AB, EM TECNOLOGIA CMOS.}

Dissertação apresentada à Escola

Politécnica da Universidade de São

Paulo para obtenção do título de Mestre em Engenharia.

Área de Concentração:

Microeletrônica

Orientador:

Prof. Dr. João Navarro Soares Jr. 
Este exemplar foi revisado e alterado em relação à versão original, sob responsabilidade única do autor e com a anuência de seu orientador.

São Paulo, 10 de Julho de 2006.

Assinatura do autor

Assinatura do orientador

Franco Neto, Murillo F.

Técnica para o Projeto de um Amplificador Operacional Folded Cascode, Classe AB, em Tecnologia CMOS. São Paulo, 2006. $100 \mathrm{p}$.

Dissertação (Mestrado) - Escola Politécnica da Universidade de São Paulo. Departamento de Sistemas Eletrônicos.

1. Circuitos Integrados CMOS 2.Amplificadores I. Universidade de São Paulo. Escola Politécnica. Departamento de Sistemas Eletrônicos. II t. 


\section{Agradecimentos}

Ao meu orientador Prof. Dr. João Navarro Soares Jr. e ao Prof. Dr. Wilhelmus Van Noije, pela insistência, paciência e pelas inúmeras críticas, sempre me levando ao máximo. Aos amigos Gustavo Vásquez e Andrés Farfán, doutorandos do LSI, por estarem sempre presentes quando mais precisei. Aos meus pais Murillo Franco Filho e Margarida Franco, meu irmão Marcelo Franco, à minha namorada Paula Goulart, à minha avó Maria Emília e meus tios José Augusto Taveira e José Geraldo Taveira, por me darem força e apoio, algo inestimável. Sem eles este trabalho não existiria. 
"Toda tristeza humana provém da diferença entre o mundo real $e$ o mundo imaginário"

\section{- Platão}




\section{Sumário}

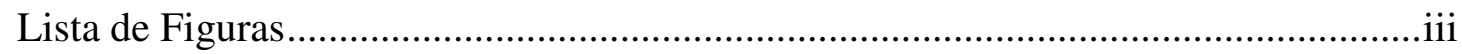

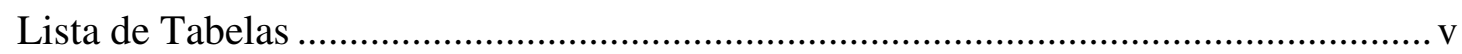

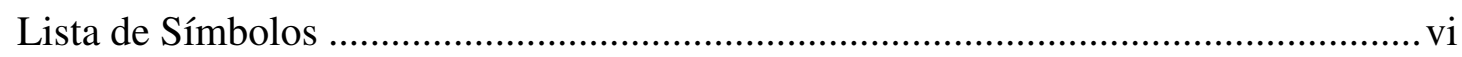

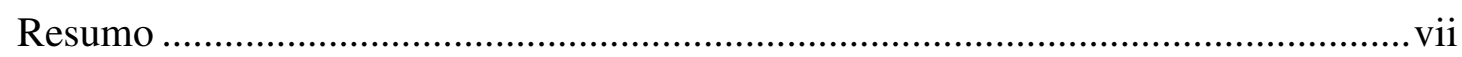

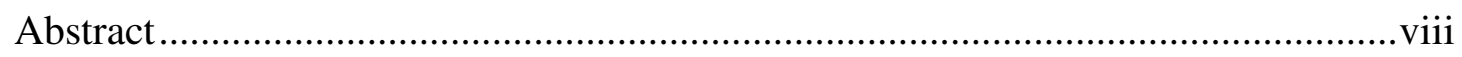

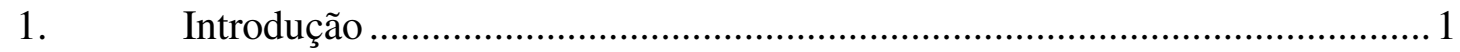

2. O Amplificador Operacional Simples......................................................

2.1. Projeto do Amplificador Operacional Simples …............................................... 3

3. O Amplificador Operacional Folded Cascode............................................ 14

3.1. Descrição Funcional do ampOp Folded Cascode ............................................. 19

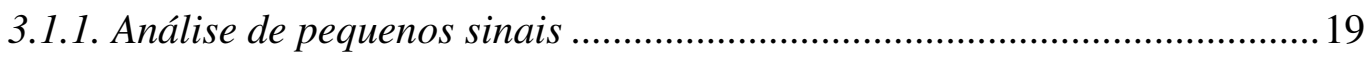

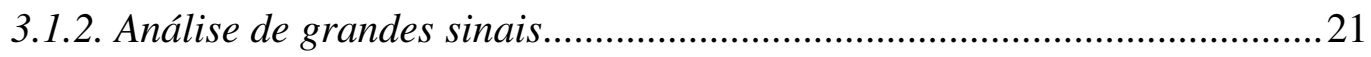

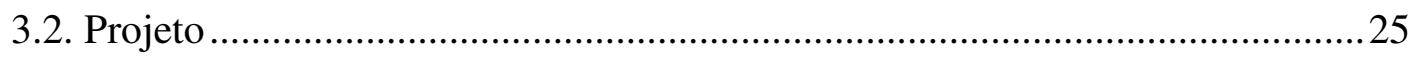

3.2.1. Projeto dos transistores MN e MP do estágio de saída............................25

3.2.2. Cálculo das tensões $\operatorname{Vgs}_{M N}$ e $V g s_{M P}$ (quiescentes) nos terminais de porta

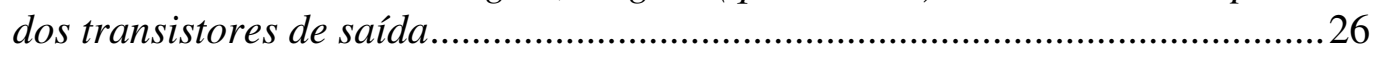

3.2.3. Projeto dos capacitores, $C_{C}$, e resistores, $R_{C}$, de compensação ................22

3.2.4. Projeto dos transistores de entrada M1 e M2 .........................................22

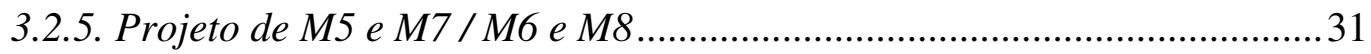

3.2.6. Projeto do circuito de controle AB, composto por M15 e M16 ..................33

3.2.7. Projeto das fontes de corrente M3-M4, M9-M10 e M11-M12 ..................36

3.2.8. Projeto do estágio de polarização …….................................................... 37

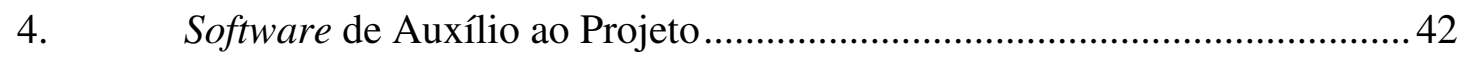

4.1 Análise do Software de auxílio ao projeto ......................................................45

4.1.1 Análise da rotina designCheck(Amplifier *thisAmplifier)........................47 


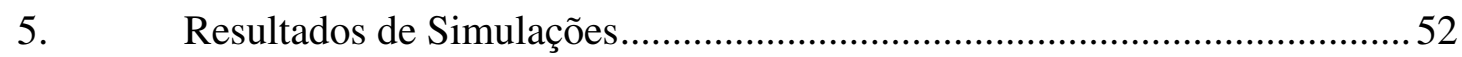

5.1 Input Common Mode Range - ICMR ….................................................. 52

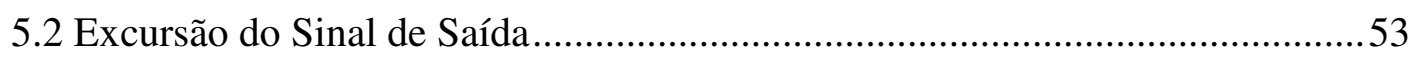

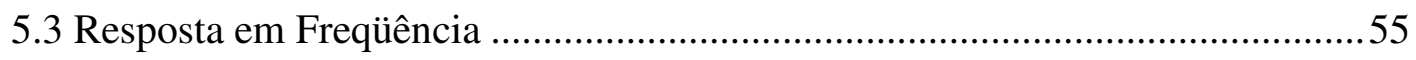

5.4 Common Mode Rejection Ratio - CMRR ..................................................56

5.5 Power Supply Rejection Ratio - PSRR ...................................................57

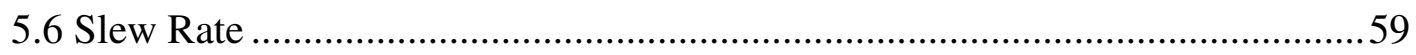

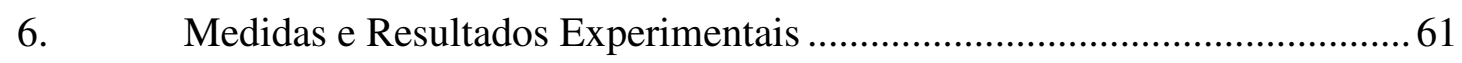

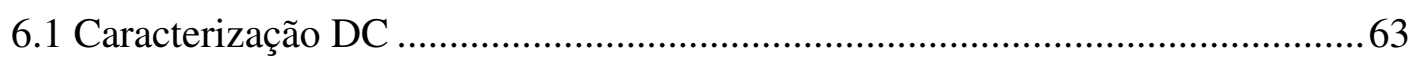

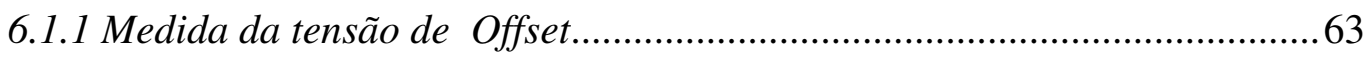

6.1.2 Medida de Input Common Mode Range - ICMR ......................................64

6.1.3 Medida de Excursão do Sinal de Saída .....................................................66

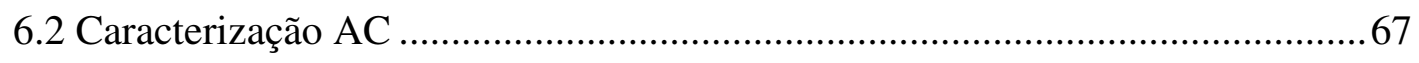

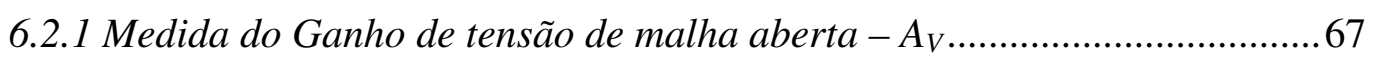

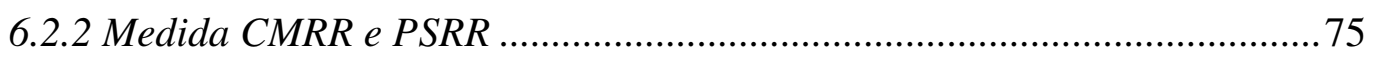

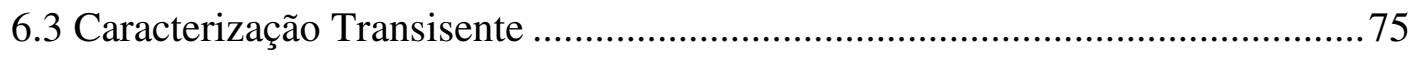

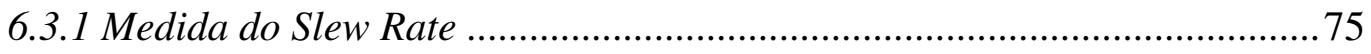

6.3.2 Medida da Margem de Fase ................................................................ 77

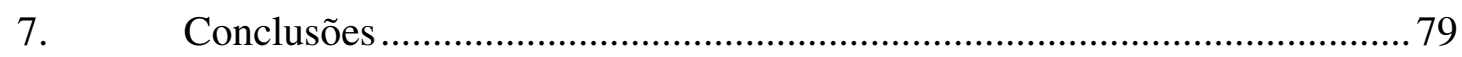

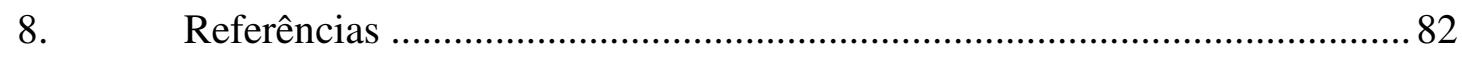

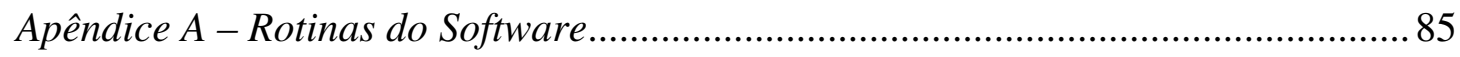




\section{Lista de Figuras}

Figura 2.1. Esquemático do amplificador simples....................................................

Figura 2.2. Disposição dos transistores do amplificador simples, para realização do

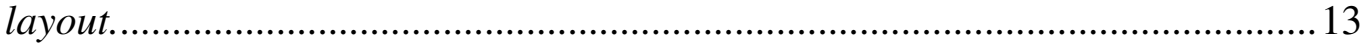

Figura 3.1. Topologia completa do amplificador operacional folded cascode .............16

Figura 3.2. Testbench utilizado para a simulação do amplificador operacional folded

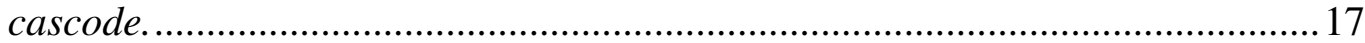

Figura 3.3. Circuito simplificado que modela o amplificador operacional folded

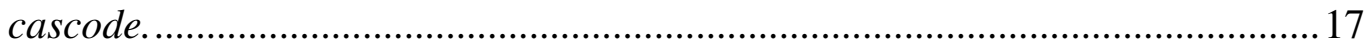

Figura 3.4. Testbench utilizado para simulação de grandes sinais.............................2 21

Figura 3.5. a) Degrau aplicado à entrada $I N$ do ampOp e b) sinal na saída do ampOp.

Figura 3.6. a) Corrente injetada pela fonte $M 3-M 4$ que polariza o par diferencial de entrada b) Corrente de dreno do transistor M7.

Figura 3.7. a) Corrente de dreno de M8. b) Tensão no terminal de fonte de $M 15$ (terminal de porta de $M N$ ). c) Tensão no terminal de fonte de $M 16$ (terminal de porta de $M P$ ). d) Corrente injetada pela fonte formada por $M 11-M 12 \ldots \ldots \ldots \ldots . . .24$

Figura 4.1. Objeto AO composto de outros objetos................................................42

Figura 4.2. Hierarquia entre os objetos criados na linguagem $\mathrm{C}$ para modelar o Amplificador Operacional Folded Cascode ....................................................43

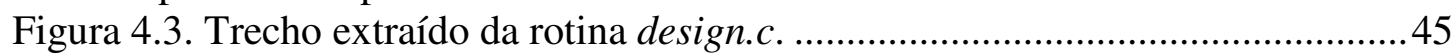

Figura 4.4. Trecho extraído da rotina design.c. ...........................................................46

Figura 4.5. Diagrama de estados da rotina designCheck( )........................................48

Figura 5.1. Testbench utilizado para simulação e medida de ICMR ............................52

Figura 5.2. Simulação de ICMR do amplificador folded cascode para o caso típico, 40 ${ }^{\circ} \mathrm{C}, I_{B I A S}=1,5 \mu A$.

Figura 5.3. Testbench utilizado para simulação e medida de excursão do sinal de saída.

Figura 5.4. Simulação da excursão de sinal de saída para o caso típico, $40^{\circ} \mathrm{C}, I_{B I A S}=$ $1,5 \mu A$.

Figura 5.5. Testbench utilizado para simulação da resposta em freqüência do amplificador. 55

Figura 5.6. Resultado da simulação AC do circuito da figura 5.5, para o caso típico, $40^{\circ} \mathrm{C}, I_{B I A S}=1,5 \mu A$.

Figura 5.7. Testbench utilizado para simulação de $C M R R$.........................................56

Figura 5.8. Resultado da simulação para verificação de $C M R R$, para o caso típico, 40

${ }^{\circ} \mathrm{C}, I_{B I A S}=1,5 \mu A$.

Figura 5.9. Testbench utilizado para simulação de $P S R R$...........................................58

Figura 5.10. Resultado da simulação de $P S R R$, para o caso típico, $40^{\circ} \mathrm{C}, I_{B I A S}=$

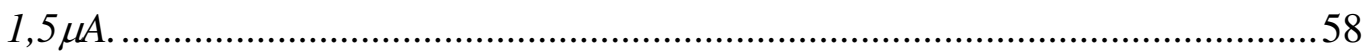

Figura 5.11. Testbench utilizado para simulação e medida de slew rate ....................59

Figura 5.12. Pulso de entrada e resposta do circuito, para verificação do $S R$, para o

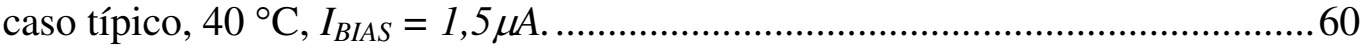

Figura 6.1. Fotomicrografia do ampOp folded cascade fabricado. .............................61

Figura 6.2. Layout do ampOp folded cascode. Os transistores estão identificados.....62 
Figura 6.3. Testbench utilizado para medida da tensão de offset de entrada do ampOp.

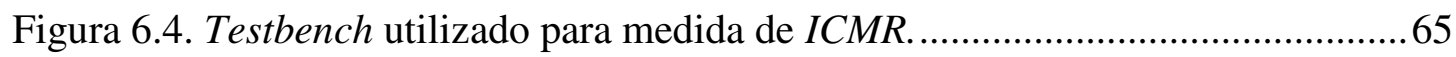

Figura 6.5. ICMR medido do amplificador folded cascode .......................................65

Figura 6.6. Testbench utilizado para medida de excursão do sinal de saída.................66

Figura 6.7. Medida da excursão de sinal de saída do amplificador. ............................67

Figura 6.8. Tensões necessárias para o cálculo do ganho de malha aberta do amplificador operacional.

Figura 6.9. Testbench utilizado para medida do ganho de malha aberta. ...................68

Figura 6.10. Amplificador de Instrumentação (in-amp) utilizado para amplificação do

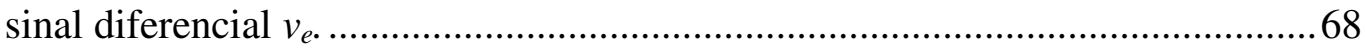

Figura 6.11. Ganho do amplificador de instrumentação em função da freqüência do

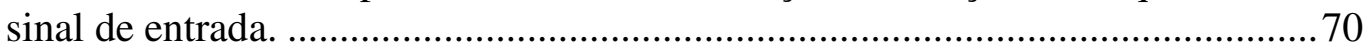

Figura 6.12. Capacitor $C$ introduzido para possibilitar a amplificação de $v_{e}$, eliminando o offset da medida realizada........................................................ 72

Figura 6.13. Simulação do módulo da resposta em frequiência do amplificador, utilizando um capacitor de $220 \mu \mathrm{F}$ de acoplamento na entrada negativa, conforme

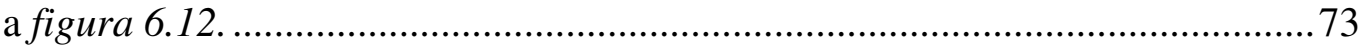

Figura 6.14. Módulo da resposta em freqüência do circuito da figura 6.12 ................74

Figura 6.15. Testbench utilizado para medida de slew rate e margem de fase.............76

Figura 6.16. Resposta transiente, ilustrativa, do circuito à um pulso quadrado na entrada. 


\section{Lista de Tabelas}

Tabela 2.1. Limites de operação de um transistor MOS 7

Tabela 2.2. Estimativa do parâmetro $\lambda$, que representa o inverso da tensão de Early 11

Tabela 2.3. Comparação entre projeto e simulação do amplificador operacional simples.

Tabela 3.1. Especificações para o projeto do amplificador operacional folded cascode.

Tabela 3.2 Constantes Físicas.

Tabela 3.3. Transistores projetados para o ampOp folded cascode .............................41

Tabela 4.1. Relação entre os objetos que formam o amplificador e os arquivos de código.

Tabela 4.2. Características escolhidas para serem modificados durante o reprojeto do amplificador.

Tabela 5.1. Parâmetros retirados do gráfico da simulação AC do amplificador. .........55

Tabela 5.2. Valores de $C M R R$ extraídos do gráfico da figura 5.8 ...............................57

Tabela 5.3. Valores de PSRR extraídos do gráfico da figura 5.10...............................59

Tabela 5.4. Valores de $S R$ extraídos do gráfico da figura 5.12 ..................................6 60

Tabela 6.1. Valores medidos das tensões do circuito para cálculo da tensão de offset

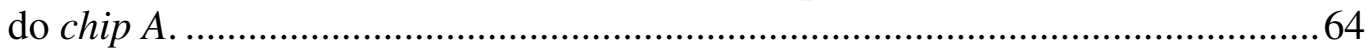

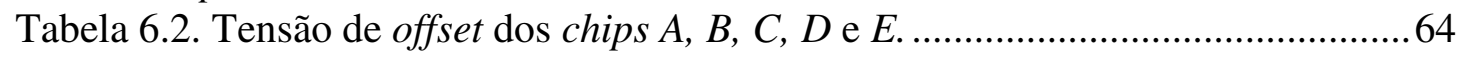

Tabela 6.3. Resistores utilizados na montagem do testbench da figura 6.6. ...............66

Tabela 6.4. Valores dos resistores que formam o amplificador de instrumentação. ...69

Tabela 6.5. Valores medidos para o ganho do in-amp em função da freqüência........ 70

Tabela 6.6. Valores escolhidos para os componentes da figura 6.12 .........................72

Tabela 6.7. Valores retirados da figura 6.13, caracterizando o módulo da resposta em frequiência do amplificador com capacitor de acoplamento na entrada negativa. 74

Tabela 6.8. Medidas retiradas do gráfico da figura 6.14 ........................................... 75

Tabela 7.1. Comparação entre os resultados projetados, simulados e medidos do ampOp. 


\section{Lista de Símbolos}

$\begin{array}{ll}g m_{M i} & \text { transcondutância do transistor } \boldsymbol{i} \\ g m b_{M i} & \text { transcondutância devido ao efeito de corpo do transistor } \boldsymbol{i} \\ g m_{O} & \text { transcondutância do estágio de saída } \\ g d s_{M i} & \text { condutância do transistor } \boldsymbol{i} \\ R_{O i} & \text { impedância total no nó } \boldsymbol{i} \\ C_{i} & \text { capacitância total no nó } \boldsymbol{i} \\ C_{g d i} & \text { capacitância entre os terminais de porta e dreno do transistor } \boldsymbol{i} \\ C_{g s i} & \text { capacitância entre os terminais de porta e fonte do transistor } \boldsymbol{i} \\ C_{d b i} & \text { capacitância entre os terminais de dreno e corpo do transistor } \boldsymbol{i} \\ C_{S i d e w a l l i} & \text { capacitância das paredes laterais do terminal de dreno do transistor } \boldsymbol{i} \\ C_{J u n c t i o n i} & \text { capacitância de junção do terminal de dreno do transistor } \boldsymbol{i} \\ C_{o x} & \text { capacitância do óxido de porta do transistor MOS } \\ M F=\Phi_{M} & \text { margem de fase } \\ f p_{i} & \text { frequência do pólo } \boldsymbol{i} \\ f z_{i} & \text { frequência do zero } \boldsymbol{i} \\ W_{i} & \text { largura de canal do transistor } \boldsymbol{i} \\ L_{i} & \text { comprimento de canal do transistor } \boldsymbol{i} \\ d B & \text { decibel } \\ \Omega & \text { Ohm } \\ F & \text { Faraday }\end{array}$




\section{Resumo}

A tendência mundial em torno de sistemas SoC - System on Chip - baseados em processo CMOS - Complementary Metal Oxide Semiconductor - digital, apresenta cada vez mais desafios aos projetistas de circuitos integrados. Em especial se observa que enquanto os projetistas de circuitos digitais podem contar com bibliotecas cada vez mais completas de células digitais semi-prontas e ferramentas cada vez mais poderosas para o aprimoramento do projeto, os projetistas analógicos não contam com tais facilidades, sendo necessário realizar o projeto de novas células analógicas para cada especificação recebida.

Este trabalho apresenta uma contribuição para a automatização do projeto de blocos analógicos e, para isso, foi escolhido um bloco essencial em muitos projetos analógicos: o amplificador operacional - ampOp.

A idéia inicial por trás dessa escolha foi um conjunto de especificações fornecido pela empresa Freescale Semiconductors, para o projeto um préamplificador de áudio realizado no âmbito do Programa Nacional de Microeletrônica $-P N M$.

A topologia escolhida para o amplificador operacional, retirada de [1], foi analisada e utilizada para projeto do amplificador para áudio. Além disso, um software de auxílio ao projeto para este amplificador foi escrito em linguagem $C$, e seu objetivo é auxiliar no reprojeto do ampOp para atender à especificações diversas. Para isso o software recebe como entradas as próprias especificações e um primeiro projeto do ampOp, realizado com equações simplificadas de projeto. software então, em conjunto com um simulador elétrico, reprojeta o amplificador, retirando alguns parâmetros relevantes dos arquivos de simulação e utilizando equações de projeto mais completas.

Ao final do trabalho, um exemplo de ampOp foi fabricado e caracterizado, sendo os resultados obtidos analisados. 


\section{Abstract}

The world trend towards SoC - System on Chip - based on digital CMOS Complementary Metal Oxide Semiconductor - process presents more and more challenges to the IC designer. One can observe that while digital designers may rely on digital core libraries that are more and more complete, and design tools that are increasingly powerful and capable of optimizing the digital design, analog designers do not have such privileges available, becoming necessary to design such analog cores each time a new set of specifications is received.

This work presents a contribution to the automatization of the design of analog cores and, in order to do that, an essential core was chosen: the operational amplifier.

The choice for the operational amplifier was made in order to attend to a set of specifications provided by Freescale Semiconductors. This set was applied in the design of an audio pre-amplifier performed in the scope of the National Microelectronics Program - PNM.

A topology chosen for the amplifier, extracted from [1], was analysed and applied to design the audio pre-amplifier. Additionaliy, a software for this specific amplifier was written, and its goal is to aid the redesign of the amplifier to comply with a set of specifications. In order to do this, the software receives, as input parameters, the set of specifications and the results of a first amplifier design, done by the analog designer using simplified equations. Then, together with an electrical simulator, the software redesigns the amplifier, reading some relevant information from the output file of the simulation and using more complete relations.

At the end of this work, an example of amplifier was manufactured and characterized, and the final results were analyzed. 


\section{Capítulo 1}

\section{Introdução}

Os projetistas de circuitos integrados digitais têm, como um grande auxílio ao projeto, a disponibilidade de células digitais prontas para uso. Por exemplo, os FPGAs (Field Programmable Gate Arrays) são dispositivos de lógica programável que oferecem inúmeras funcionalidades semi-prontas, como contadores, registradores de deslocamento e até mesmo células de memória RAM. Isso facilita e agiliza o projeto de sistemas uma vez que basta ao projetista escolher quais células atendem melhor aos seus requisitos, realizando, se necessário, pequenas modificações às suas funcionalidades, e elas estarão prontas para serem utilizadas.

O projetista de circuitos analógicos também pode contar com algumas células prontas, mas não conta com as mesmas facilidades. Por exemplo, ao modificar uma característica de um amplificador operacional (ampOp), como o ganho em malha aberta, alterando a transcondutância dos transistores do par diferencial de entrada, outras características do circuito estarão também sendo modificadas, como banda, ruído e consumo, para indicar algumas. Dessa maneira, o projetista analógico constantemente depara com situações onde, ao se melhorar uma característica do circuito, outras são degradadas. Na literatura, a relação entre a troca é conhecida como trade-off.

Uma outra diferença importante, que deve ser levado em conta pelo projetista analógico, diz respeito à redução das dimensões mínimas dos transistores fabricados em uma dada tecnologia. Conforme o comprimento do canal de transistores CMOS diminui, efeitos de segunda ordem, como modulação de canal, começam a se tornar mais relevantes e as equações quadráticas, que descrevem o comportamento do transistor em primeira aproximação, começam a fornecer resultados muito imprecisos [4].

Com a tendência mundial de integração de sistemas completos em um mesmo chip (SoC - System on Chip), se torna essencial que os diversos blocos, tanto digitais como analógicos, possam ser obtidos da forma mais simples e rápida possível. Além disso, no caso analógico, é importante que os resultados do projeto levem em conta os efeitos de segunda ordem, para que os valores projetados de banda, margem de fase, ganho, etc. se aproximem ao máximo dos valores encontrados na simulação e no circuito real. 
Por essas razões, torna-se importante a existência de ferramentas computacionais que auxiliem o projetista no projeto inicial do circuito, bem como blocos analógicos prontos, que possam ser utilizados no mundo analógico de maneira semelhante a aquela que blocos digitais já são utilizados no mundo digital. Este trabalho irá apresentar uma tentativa de se utilizar a metodologia do mundo digital no mundo analógico. Para tal, será projetado um amplificador operacional, ampOp., folded cascode, com estágio de saída classe AB. Este amplificador de dois estágios será parametrizado e, junto com essa tarefa, será desenvolvido um software de auxílio ao projeto deste amplificador, cujo objetivo é aumentar a velocidade de projeto do ampOp. Para a utilização deste software, o projetista deverá entrar com as especificações de projeto, e o software, em conjunto com um simulador elétrico, realizará a determinação das dimensões do circuito, entregando um arquivo de netlist do amplificador. O amplificador operacional projetado será também fabricado na tecnologia CMOS $0.35 \mu \mathrm{m}$, da AMS, e caracterizado.

Este trabalho de mestrado se encontra dividido da seguinte maneira: 0 Capítulo 2 apresenta o projeto de um amplificador operacional simples, amplamente conhecido na literatura, onde se aplica a metodologia de projeto proposta de forma a se identificar eventuais problemas (como por exemplo extração de parâmetros de pequenos sinais dos transistores MOS) e corrigí-los, uma vez que os resultados obtidos podem ser prontamente analisados.

O Capítulo 3 analisa a topologia folded cascode extraída de [1], apresentando uma sequência para o projeto do ampOp e fornece as dimensões $W / L(W=$ largura, $L=$ comprimento do canal $)$ dos transistores projetados.

O Capítulo 4 apresenta o software de auxílio ao projeto, numa primeira tentativa de se implementar a sequência proposta no capítulo 3 numa linguagem de programação, e a máquina de estados finita (FSM - Finite State Machine) que descreve os estados pelos quais o reprojeto do ampOp passará até atender às especificações.

O Capítulo 5 mostra os resultados de simulação do amplificador folded cascode projetado, enquanto que o Capítulo 6 apresenta os resultados de medidas realizadas no circuito fabricado.

Finalmente o Capítulo 7 consolida os resultados de projeto, simulação e medidas, apresentando algumas comparações e conclusões. Seguem-se então a bibliografia utilizada e um apêndice contendo as rotinas computacionais implementadas para o software de auxílio ao projeto. 


\section{Capítulo 2}

\section{O Amplificador Operacional Simples}

A técnica aplicada ao projeto do amplificador operacional folded cascode foi, inicialmente, aplicada ao projeto de um amplificador operacional mais simples, o que é apresentado neste capítulo.

\subsection{Projeto do Amplificador Operacional Simples}

O amplificador simples é apresentado na figura 2.1. Ele é composto de um par diferencial com transistores tipo NMOS na entrada, tendo como carga um espelho de corrente com transistores tipo PMOS [3]. A fonte de corrente é implementada através de um espelho NMOS, que espelha uma corrente de polarização fornecida por algum circuito externo. Finalmente, foi considerada uma capacitância de carga $C_{L}=10 \mathrm{pF}$.

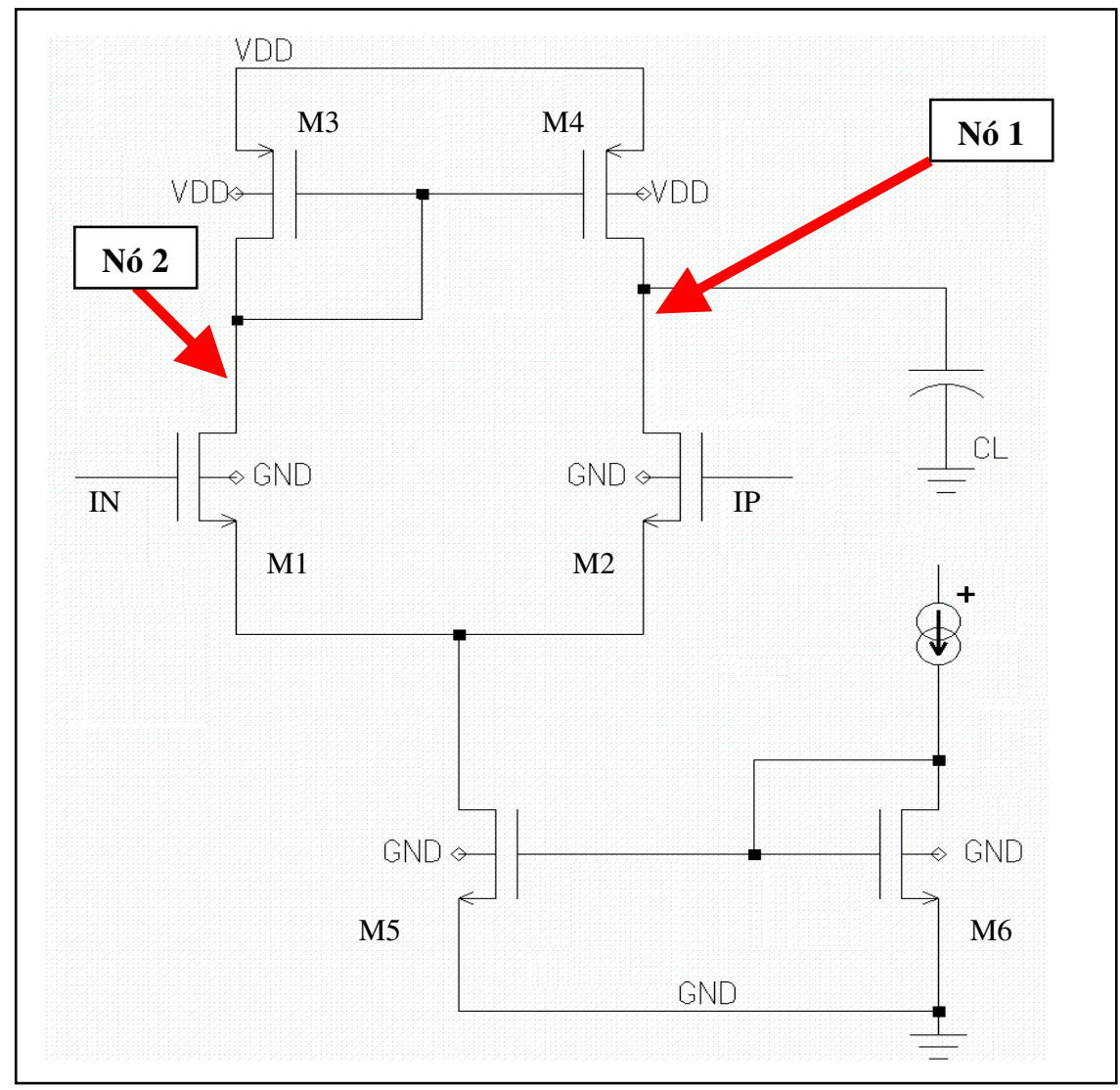

Figura 2.1. Esquemático do amplificador simples. 
O projetista deve utilizar as expressões mais próximas possível das expressões utilizadas pelo simulador sem, no entanto, torná-las complexas demais a ponto de dificultar um primeiro projeto. Infelizmente, com a diminuição do tamanho do canal dos transistores na tecnologia CMOS, diversos efeitos de segunda ordem começam a se tornar cada vez mais importantes, o que faz com que a expressão quadrática dos transistores MOS seja cada vez mais uma aproximação grosseira [4]. Entretanto, para um primeiro projeto, normalmente o projetista não tem como estimar com precisão alguns termos importantes, como por exemplo gds ou gm, que são necessários para se utilizar expressões mais completas. Por essa razão, neste capítulo o projeto do ampOp simples será realizado utilizando-se expressões simplificadas e, quando necessário, os parâmetros de pequenos sinais serão estimados. No Capítulo 3 uma metodologia mais elaborada será apresentada, onde esses parâmetros não são estimados, mas sim retirados dos arquivos de simulação.

Alguns termos utilizados nas equações de projeto abaixo são definidos a seguir:

$g m_{M i}$ é a transcondutância do transistor $\boldsymbol{i}$;

$g m b_{M i}$ é a transcondutância devido ao efeito de corpo do transistor $i$;

$g m_{O}$ é a transcondutância do estágio de saída;

$g d s_{M I}$ é a condutância do transistor $\boldsymbol{i}$;

$R_{O i}$ é a impedância total no nó $i$;

$C_{i}$ é a capacitância total no nó $\boldsymbol{i}$;

$C_{g d i}$ é a capacitância entre os terminais de porta e dreno do transistor $\boldsymbol{i}$;

$C_{g s i}$ é a capacitância entre os terminais de porta e fonte do transistor $\boldsymbol{i}$;

$C_{d b i}$ é a capacitância entre os terminais de dreno e corpo do transistor $\boldsymbol{i}$;

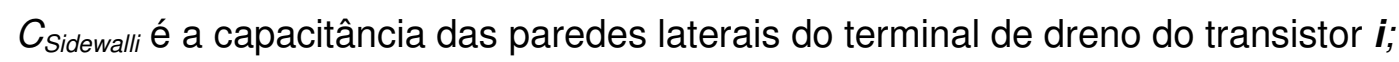

$C_{\text {Junctioni }}$ é a capacitância de junção do terminal de dreno do transistor $\boldsymbol{i}$;

$C_{o x}$ é a capacitância do óxido de porta do transistor MOS;

$M F=\Phi_{M}$ é a margem de fase;

$f p_{i}$ é a frequência do pólo devido ao nó $\boldsymbol{i}$;

$f z_{i}$ é a frequência do zero devido ao nó $\boldsymbol{i}$;

$W_{i}$ é a largura de canal do transistor $\boldsymbol{i}$;

$L_{i}$ é a comprimento de canal do transistor $\boldsymbol{i}$. 
Vamos determinar as expressões simplificadas que serão utilizadas no projeto do amplificador simples. A corrente de dreno dos transistores na região de saturação pode ser aproximada pela relação quadrática,

$$
I_{D}=\frac{1}{2} \cdot K \cdot \frac{W}{L} \cdot\left(V_{G S}-V_{T H}\right)^{2} \cdot\left(1+\lambda \cdot V_{D S}\right)
$$

onde,

Ké o fator de ganho do transistor MOS, em $\mu A / V^{2}$;

We $L$ são o largura e o comprimento do canal;

$V_{G S}$ é a tensão porta fonte;

$V_{T H}$ é a tensão de limiar do transistor MOS;

$V_{D S}$ é a tensão dreno fonte;

$\lambda$ é o inverso da tensão de Early.

Essa expressão leva em consideração o efeito de modulação de canal, bastante importante em transistores de canal curto. Ela apresenta, porém, o termo $\lambda$ que é difícil de ser estimado, conforme será explicado neste capítulo. Por esse motivo, a expressão acima será reduzida e o termo $\left(1+\lambda \cdot V_{D S}\right)$ não será considerado, o que fornece,

$$
I_{D}=\frac{1}{2} \cdot K \cdot \frac{W}{L} \cdot\left(V_{G S}-V_{T H}\right)^{2}
$$

Outra expressão importante é a que modela a tensão de saturação dos transistores, dada pela relação,

$$
V_{D S A T}=\sqrt{\frac{2 \cdot I_{D}}{K \cdot \frac{W}{L}}}
$$

Podemos verificar que a resistência total vista a partir do nó 1 do circuito da figura 2.1 é dada por,

$$
R_{o 1}=\frac{1}{g d s_{M 4}} \| \frac{1}{g d s_{M 2}}
$$

e a resistência vista a partir do nó 2 pode ser expressa por,

$$
R_{o 2}=\frac{1}{g m_{M 3}} \| \frac{1}{g d s_{M 1}} \approx \frac{1}{g m_{M 3}}
$$

A capacitância total, vista a partir do nó 1, pode ser aproximada por,

$$
C_{1} \approx C_{L}
$$

pois a capacitância de carga $C_{L}$ de 10 pF é muito maior que eventuais capacitâncias internas. Porém, a capaciância total no nó 2 é formada exclusivamente por capacitâncias internas, podendo ser expressa através de, 


$$
C_{2}=C_{g d 1}+C_{d b 1}+C_{g s 3}+C_{d b 3}+C_{g s 4}+A_{V M 4} \cdot C_{g d 4}
$$

onde $A_{V M 4}$ representa o ganho de tensão do transistor $M 4$, e o termo $A_{V M 4} \cdot C_{g d 4}$ se deve ao efeito Miller (a capacitância entre os terminais de porta e dreno do transistor é refletida para seu terminal de porta, multiplicada pelo fator $\left(1+A_{V M}\right) \approx A_{V M}$ ) [8], [9], [14]. Na saturação, as capacitâncias de um transistor MOS são dadas, aproximadamente, por,

$$
C_{g s}=\frac{2}{3} \cdot C_{o x} \cdot W \cdot L \quad \text { e } \quad C_{g d}=0
$$

sendo $C_{g d}$ a capacitância intrínseca. Logo, substituindo $C_{g d}=0$ em (2.6):

$$
C_{2}=C_{d b 1}+C_{g s 3}+C_{d b 3}+C_{g s 4}
$$

Essas capacitâncias podem ser estimadas e são, a princípio, muito menores do que a capacitância de carga $C_{L}$. Dessa maneira, vamos assumir que o pólo devido ao nó 2 está numa frequência muito elevada e o amplificador simples tem um pólo dominante dado por,

$$
f p_{1}=\frac{1}{2 \cdot \pi \cdot R_{o 1} \cdot C_{1}} \approx \frac{1}{2 \cdot \pi \cdot R_{o 1} \cdot C_{L}}
$$

Já o pólo não dominante é determinado por,

$$
f p_{2}=\frac{1}{2 \cdot \pi \cdot R_{o 2} \cdot C_{2}}
$$

onde $C_{2}$ é dado por (2.8).

O ganho DC do circuito é dado pela transcondutância do transistor de entrada, M1, multiplicada pela impedância total do nó 1, dada por (2.3). Assim,

$$
A_{V}=g m_{M 1} \cdot R_{o 1}
$$

Uma vez que o transistor MOS esteja operando na região de saturação, sua transcondutância gm pode ser calculada de acordo com a quantidade de portadores presentes no canal, formado sob o terminal de porta. Devemos levar em consideração, portanto, se o transistor está operando em inversão forte, inversão fraca ou inversão moderada, sendo elas:

- Inversão Forte (Strong Inversion): a tensão $V_{G S}$ (porta-fonte) é superior à tensão de limiar, o que permite que um canal bem definido seja formado na região de depleção abaixo do terminal de porta. A corrente obedece a relação quadrática (2.1) (sem considrear efeitos de canal curto);

- Inversão Fraca (Weak Inversion): a tensão $V_{G S}$ (porta-fonte) está em torno da tensão de limiar, sendo suficiente para formar uma região de depleção 
mas insuficiente para formar um canal bem definido. A corrente obedece uma relação exponencial;

- Inversão Moderada (Moderate Inversion): é a região de transição, não muito bem demarcada, entre a região de inversão forte e a região de inversão fraca.

Um critério para determinar em qual região o transistor está operando é dado pela tabela $2.1[5]$ :

Tabela 2.1. Limites de operação de um transistor MOS.

\begin{tabular}{|c|c|}
\hline Região de Operação & Condição \\
\hline Inversão Forte & LIM $>8$ \\
\hline Inversão Fraca & LIM $<0,125$ \\
\hline Inversão Moderada & $0,125<$ LIM $<8$ \\
\hline
\end{tabular}

O parâmetro LIM é calculado através de,

$$
L I M=\frac{I_{D}}{I_{D \lim }}
$$

e,

$$
I_{D \lim }=K \cdot \frac{W}{L} \cdot 2 n \cdot U_{T}^{2}
$$

onde,

$I_{D}$ é a corrente de dreno do transistor MOS.

$n$ é o fator de inclinação de inversão fraca, que pode ser aproximado por $n=\left(1+\frac{1}{K}\right)[5]$, onde $K$ é dado em $\mu A / V^{2}$.

$U_{T}=\frac{k \cdot T}{q} \approx 27 m V$, considerando a temperatura ambiente igual à $40{ }^{\circ} \mathrm{C}$.

ké a constante de Boltzmann $=1,38 \times 10^{-23}$ joules $/$ kelvin.

$T$ é a temperatua absoluta em kelvin $=273+$ temperatura em $\left({ }^{\circ} \mathrm{C}\right)$.

$q$ é a magnitude da carga do elétron $=1,60 \times 10^{-19}$ coulomb.

Uma vez determinada a região de operação do transistor de entrada, a transcondutância é definida pelas relações abaixo: 
- Inversão Forte (LIM > 8)

$$
g m \approx \sqrt{2 \cdot I_{D} \cdot K \cdot \frac{W}{L}}=\frac{2 \cdot I_{D}}{V_{G S}-V_{T H}}
$$

- Inversão Fraca (LIM $<0,125)$

$$
g m \approx \frac{I_{D}}{n \cdot U_{T}}
$$

- Inversão Moderada $(0,125<\operatorname{LIM}<8)$

$$
g m \approx \frac{I_{D}}{n \cdot U_{T} \cdot \sqrt{1+L I M}}
$$

Como foi visto anteriormente, o segundo pólo, dado por (2.10), se encontra numa frequência muito maior do que a do primeiro pólo (2.9). Assim, a banda de ganho unitário do circuito, GBW (Gain-Bandwidth product), pode ser determinada [14] através da expressão:

$$
G B W=A_{V} \cdot f p_{1}
$$

Um outro parâmetro importante do amplificador é a margem de fase (MF). Ela é responsável pela estabilidade do sistema, influenciando diretamente no settling time da resposta transiente [8]. O settling time é o tempo que o sinal na saída levará para se estabilizar em torno do valor final de tensão. Valores muito baixos de margem de fase implicam em um tempo de subida pequeno e em um settling time grande, onde o sinal de saída pode até oscilar. Por outro lado, valores elevados de margem de fase implicam em um tempo de subida grande e em um settling time pequeno.

De forma genérica, a margem de fase de um sistema pode ser calculada da seguinte maneira:

$$
\Phi_{M}=180^{\circ}+\arctan \left(\frac{f}{f z_{1}}\right)+\ldots+\arctan \left(\frac{f}{f z_{n}}\right)-\arctan \left(\frac{f}{\left|f p_{1}\right|}\right)-\ldots-\arctan \left(\frac{f}{\left|f p_{n}\right|}\right)
$$

onde $f z_{1} \ldots f f z_{n}$ e $f p_{1} \ldots f p_{n}$ representam as frequências dos zeros e pólos, respectivamente, do sistema, e $f$ representa uma frequência onde o ganho do circuito é $1(0 d b)$.

Para a determinação da margem de fase do amplificador aqui considerado é importante levar em consideração a posição dos dois pólos. Dessa maneira, de (2.18) teremos:

$$
\Phi_{M}=180^{\circ}-\arctan \left(\frac{f}{\left|f p_{1}\right|}\right)-\arctan \left(\frac{f}{\left|f p_{2}\right|}\right)
$$


Considerando agora que a frequência $f$ é a frequência onde o ganho é unitário,

$$
f=G B W
$$

e, de (2.17),

$$
f p_{1}=\frac{G B W}{A_{V}}
$$

substituindo (2.20) e (2.21) em (2.19):

$$
\Phi_{M}=180^{\circ}-\arctan \left(A_{V}\right)-\arctan \left(\frac{G B W}{\left|f p_{2}\right|}\right)
$$

Para um ganho $A_{v}$ grande, podemos considerar $\arctan \left(A_{V}\right)$ aproximadamente igual a $90^{\circ} \mathrm{e}$, portanto,

$$
\Phi_{M}=90^{\circ}-\arctan \left(\frac{G B W}{f p_{2}}\right)
$$

que é equivalente à:

$$
\Phi_{M}=\arctan \left(\frac{f p_{2}}{G B W}\right)
$$

A expressão (2.22) pode ser utilizada para "amarrar" a frequência de ganho unitário $G B W$ com a frequência do segundo pólo $f p_{2}$ para uma dada margem de fase. Consideremos, como exemplo, a seguinte especificação para o amplificador da figura 2.1:

$$
A_{V}=45 d B \quad M F>80^{\circ} \quad G B W>30 M H z \quad I_{T A L L}=500 \mu A
$$

sendo $I_{\text {TAlL }}$ a corrente de polarização do circuito.

O projeto do amplificador simples foi realizado utilizando rotinas implementadas na ferramenta MATLAB, que aplicam as equações apresentadas anteriormente. A seguir é mostrada a sequência de projeto e uma breve descrição dessas rotinas.

1- Determinação do Espelho de Polarização: O primeiro passo é adotar um valor para a tensão $V_{D S A T}$ do transistor M5. Essa escolha deve levar em consideração a mínima tensão de modo comum a ser aplicada nos transistores de entrada. Para o nosso caso, será escolhida uma tensão $V_{D S A T}$ $=0,38 \mathrm{~V}$. Dessa forma, a relação $\mathrm{W} / \mathrm{L}$ dos transistores do espelho de polarização (transistores M5 e M6) pode ser calculada. De (2.2) teremos:

$$
0,38=\sqrt{\frac{2 \cdot 500 \mu}{170 \mu \cdot \frac{W}{L}}}
$$


Obtendo-se uma relação $\left(\frac{W}{L}\right)_{M 5, M 6}=\frac{40}{1}[\mu m / \mu m]$.

2- Determinação da tensão porta-fonte no espelho de corrente: Foi escrita a rotina vgs_n_mirror() no MATLAB para determinar essa tensão. Essa rotina recebe como parâmetros a corrente de dreno $I_{D}$ de $M 6$, a tensão de limiar $V_{T H}$ de $M 6$, e as dimensões $W$ e $L$, que foram calculadas para o espelho de corrente no passo 1 , e retorna o valor da tensão entre os terminais portafonte de M6. Para nosso caso temos:

>>vgs_n_mirror(500e-6,0.4979,40,1)

resultando

$>\mathrm{V}_{\mathrm{GS}}=0,97 \mathrm{~V}$

3- Determinação da tensão dreno-fonte no transistor $M 5$ do espelho de corrente: Para uma primeira tentativa, será adotada para a tensão $V_{D S}$ de $M 5$ o valor de $V_{G S}$ do espelho de corrente, calculado no passo 2. Essa tensão $V_{D S}$ é importante pois ela irá determinar qual será a nova tensão de limiar, $V_{T H}$, dos transistores do par diferencial de entrada (transisotres M1 e M2). Logo,

$$
>\mathrm{V}_{\mathrm{DS}}=0,97 \mathrm{~V}
$$

4- Determinação da tensão de limiar para os transistores do par diferencial da entrada: Foi escrita a rotina vt() no MATLAB para tal. Essa rotina recebe o valor da tensão fonte-substrato, $V_{B S}$, do transistor $M 1$ ou $M 2$, e retorna o valor da nova tensão de limiar $V_{T H}$. Para nosso caso temos:

$$
>\operatorname{vt}(0,97)
$$

resultando

$$
>\mathrm{V}_{\mathrm{TH}}=0,7738 \mathrm{~V}
$$

5- Determinação das dimensões dos transistores M1, M2, M3 e M4: Para isso, a rotina dsgn() foi escrita no MATLAB. Essa rotina recebe como parâmetros a corrente de dreno $I_{D}$ que passa pelo transistor $M 1$, a tensão de limiar $V_{T H}$ de $M 1$, calculada no passo 4, o ganho desejado para o amplificador (em $\mathrm{dB}$ ) e a margem de fase desejada para o circuito. Ela retorna a relação $W / L$ dos transistores $M 1, M 2, M 3$ e $M 4$, a banda do circuito (em MHz), a tensão $V_{G S}$ dos transistores $M 1$ e $M 2$, a posição do primeiro e do segundo 
pólos, $f p_{1}$ e $f p_{2}$ em $\mathrm{Hz}$, e o parâmetro $L I M$, que indica em qual região de operação que os transistores estão trabalhando (tabela 2.1). Para o cálculo da dimensão dos transistores $M 1$ e $M 2$, são utilizados o valor do ganho fornecido e a impedância de saída, no nó 1, da figura 2.1. Essa impedância, para o caso deste circuito, é dada pela expressão (2.3), onde a condutância do transistor $i$ pode ser aproximada por:

$$
g d s \approx \lambda \cdot I_{D}
$$

e o termo $\lambda$ representa o inverso da tensão de Early, $\mathrm{V}_{\mathrm{A}}$,

$$
\lambda=\frac{1}{\left|V_{A}\right|}
$$

Para se estimar os termos $g d s_{M 2}$ e $g d s_{M 4}$ de (2.3), pode-se extrapolar a curva $I_{D} \times V_{D S}$ dos transistores PMOS e NMOS em saturação e determinar o ponto onde ela cruza o eixo horizontal. Neste ponto temos a tensão de Early (ou mais corretamente, $\left.-V_{A}\right)$. Essa caracterização foi realizada, tanto para 0 PMOS quanto para o NMOS, e os resultados estão na tabela 2.2.

Tabela 2.2. Estimativa do parâmetro $\lambda$, que representa o inverso da tensão de Early

\begin{tabular}{|c|c|c|}
\hline Parâmetro & Valor & Comprimento de canal $(L)$ \\
\hline$\lambda_{\text {PMOS }}$ & $0,047 \mathrm{~V}^{-1}$ & $1 \mu \mathrm{m}$ \\
\hline$\lambda_{\text {NMOS }}$ & $0,016 \mathrm{~V}^{-1}$ & $1 \mu \mathrm{m}$ \\
\hline
\end{tabular}

Os valores de $\lambda$ estimados foram inseridos no corpo da rotina $\boldsymbol{d s g n ( ) . ~ A g o r a ~}$ já é possível efetuar os cálculos:

>> dsgn(250e-6,0.7738,45,81)

resultando

$$
\begin{aligned}
& >>\left(\frac{W}{L}\right)_{M 1, M 2}=\frac{95}{1}[\mu m / \mu m] \\
& >\left(\frac{W}{L}\right)_{M 3, M 4}=\frac{96}{1}[\mu \mathrm{m} / \mu \mathrm{m}] \\
& >>\mathrm{fp}_{1}=250 \mathrm{kHz} \\
& >>\mathrm{fp}_{2}=281,4 \mathrm{MHz} \\
& >>\mathrm{GBW}=44.5 \mathrm{MHz} \\
& >>\mathrm{V}_{\mathrm{GS}}=0,95 \mathrm{~V} \\
& >>\mathrm{LIM}=9.1
\end{aligned}
$$


6- Determinação da tensão mínima de modo comum da entrada: A tensão mínima de modo comum da entrada deve ser calculada levando-se em consideração a tensão $V_{D S A T}$ de $M 5$, utilizada no passo 1 , e a tensão $V_{G S}$ de M1, calculada no passo 5. Assim,

$$
V_{C M \min }=V_{D S A T_{-} M 5}+V_{G S_{-} M 1}=0,38 V+0,95 V=1,33 V
$$

Foi realizada uma simulação $A C$ do circuito projetado, e o resultado dessa simulação é apresentado tabela 2.3.

Tabela 2.3. Comparação entre projeto e simulação do amplificador operacional simples.

\begin{tabular}{|c|c|c|c|}
\hline Parâmetro & Projeto & Simulação & Unidade \\
\hline I $_{\text {TAlL }}$ & 500 & 500 & $\mu \mathrm{A}$ \\
\hline VDD & 3 & 3 & $\mathrm{~V}$ \\
\hline$(\mathrm{W} / \mathrm{L})_{1,2}$ & $95 / 1$ & $95 / 1$ & $\mu \mathrm{m} / \mu \mathrm{m}$ \\
\hline$(\mathrm{W} / \mathrm{L})_{3,4}$ & $96 / 1$ & $96 / 1$ & $\mathrm{~dB}$ \\
\hline Ganho DC & 45 & 42,3 & $\mathrm{kHz}$ \\
\hline fp1 & 250 & 275 & $\mathrm{MHz}$ \\
\hline GBW & 44,5 & 35,7 & $\circ$ \\
\hline MF & 81 & 86,5 & $\mathrm{orm}$ \\
\hline
\end{tabular}

Os resultados apresentados na tabela 2.3 mostram que é possível realizar o projeto utilizando um software e a sequência aqui proposta. Os resultados simulados são próximos dos resultados desejados, com excessão do parâmetro GBW. A razão para essa diferença, de aproximadamente $20 \%$ entre os valores projetado e simulado, se deve à estimativa do parâmetro $\lambda$, em (2.24), e consequentemente, de gds em (2.23). O simulador calcula e utiliza o valor de gds no ponto de operação $D C$ do circuito, e não um valor médio, como o que se obtém ao se utilizar a tangente da curva $I_{D} \times V_{D S}$, como foi feito na caracterização dos transistores. Os valores de $g d s$ estimados e aqueles usados na simulados são muito diferentes, o que acarreta erros consideráveis no cálculo da banda. Uma forma de se tentar estimar com maior precisão o valor de gds é a partir das equações do modelo utilizado para os 
transistores, neste caso o BSIM3V3. Entretanto, as relações utilizadas neste modelo são bastante complexas o que dificulta saber como, a partir delas, se pode calcular gds. Uma outra alternativa para se obter um valor mais preciso de gds é retirá-lo do arquivo de saída da simulação, alternativa que será adotada no capítulo 3.

Para o layout do amplificador simples, alguns pontos importantes devem ser considerados [12]:

- Os transistores do par diferencial de entrada deverão ser colocados na configuração par cruzado, common-centroid. Além disso, sua vizinhança imediata deve ser simétrica, para melhorar o casamento entre M1 e M2;

- Os transistores do espelho de corrente M3 e M4, também devem ser colocados na configuração par cruzado, common-centroid. Entretanto, devido ao espelho de polarização formado por M5 e M6, sua vizinhança imediata não será simétrica. A figura 2.2 ilustra o posicionamento dos transistores, para o layout.

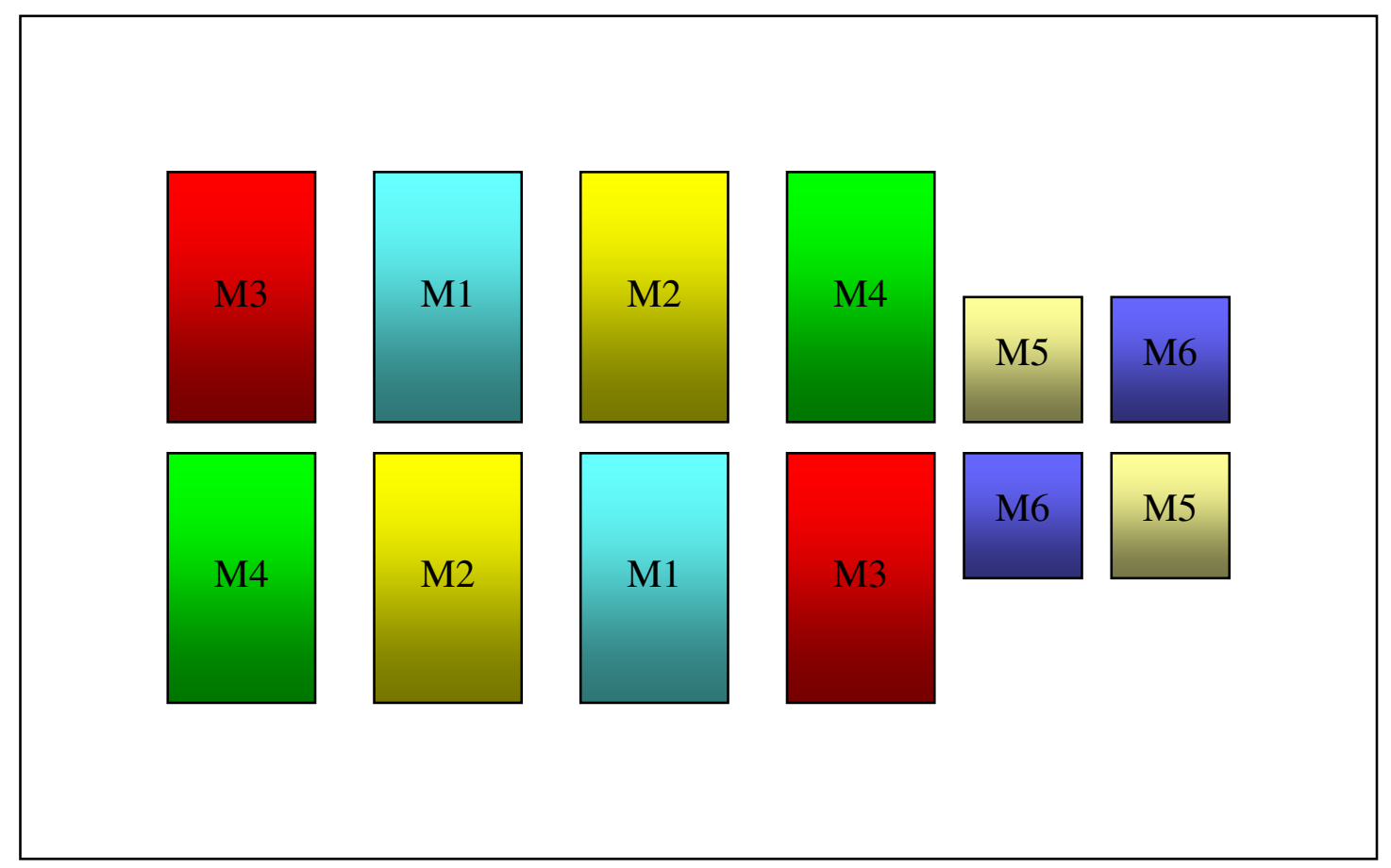

Figura 2.2. Disposição dos transistores do amplificador simples, para realização do layout. 


\section{Capítulo 3}

\section{O Amplificador Operacional Folded $\underline{\text { Cascode }}$}

O projeto do amplificador operacional folded cascode com estágio de saída classe $A B$ será apresentado nesta seção. Este é um circuito bem mais complexo do que o anterior e nele concentraremos maior atenção e esforço. A figura 3.1 mostra a topologia do amplificador.

A tabela 3.1 apresenta um conjunto de especificações que procuramos atingir para este amplificador como exemplo.

Tabela 3.1. Especificações para o projeto do amplificador operacional folded cascode.

\begin{tabular}{|c|c|c|}
\hline Especificação & Valor & Unidade \\
\hline GBW & 5 & $\mathrm{MHz}$ \\
\hline MF & 60 & $\circ$ \\
\hline$V_{\text {OUT (PP) }}$ & 2,4 & $\mathrm{~V}$ \\
\hline Slew Rate & 2 & $\mathrm{k} \Omega$ \\
\hline$R_{L}$ & 4 & $\mathrm{pF}$ \\
\hline$C_{L}$ & 30 & $\mu \mathrm{A}$ \\
\hline$I_{B I A S}$ & $1,5 \pm 20 \%$ & $\mathrm{~V}$ \\
\hline$V D D$ & 2,8 & \\
\hline
\end{tabular}

A figura 3.2 mostra como o amplificador será utilizado, em conjunto com outros componentes externos. Ele se encontra realimentado, na configuração de ganho unitário, com inversão de fase, onde $V_{\text {agnd }}=1,4 \mathrm{~V}$. O ganho dessa configuração é dado por:

$$
A_{\text {Feedback }}=-\frac{R_{1}}{R_{2}}=-1
$$

Também estão ilustradas a resistência de carga $R_{L}$ e a capacitância de carga $C_{L}$. Alguns fatores que tornam o amplificador folded cascode mais complexo que 0 amplificador simples são: 
- Estágio de polarização mais complexo;

- Estágio de saída classe AB;

- Circuito de controle de corrente mínima na saída;

- Espelhos cascode;

- Compensação Miller com resistor de anulamento de zero (nulling resistor);

- Baixo consumo;

- Baixa tensão de alimentação;

- Carga capacitiva elevada.

Além de ser mais complexo que o ampOp anterior, a literatura existente para o ampOp foldeld cascode se retringe a apenas alguns trabalhos publicados, como artigos e dissertações, que tratam de alguma característica específica do circuito. Por essa razão, algumas expressões encontradas nesses trabalhos são muito simples e não fornecem resultados adequados de projeto. Assim, para se encontrar uma expressão mais completa para, por exemplo, GBW, foi necessário o uso de uma ferramenta computacional chamada Derive 5, capaz de solucionar analiticamente o sistema de equações formado por (3.1) e (3.2), que modelam o amplificador. A figura 3.3 mostra o circuito simplificado que representa o ampOp. 


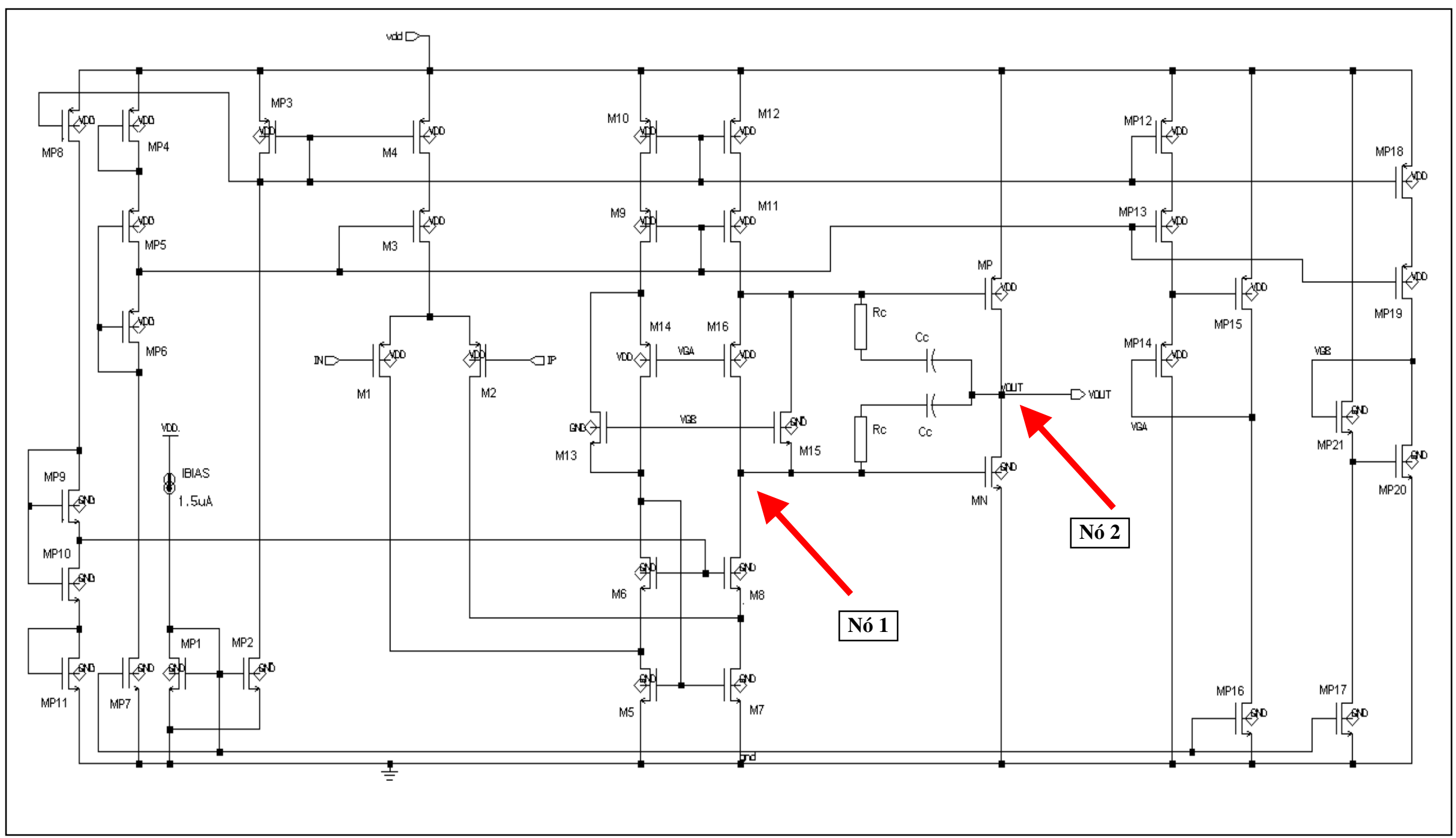

Figura 3.1. Topologia completa do amplificador operacional folded cascode. 


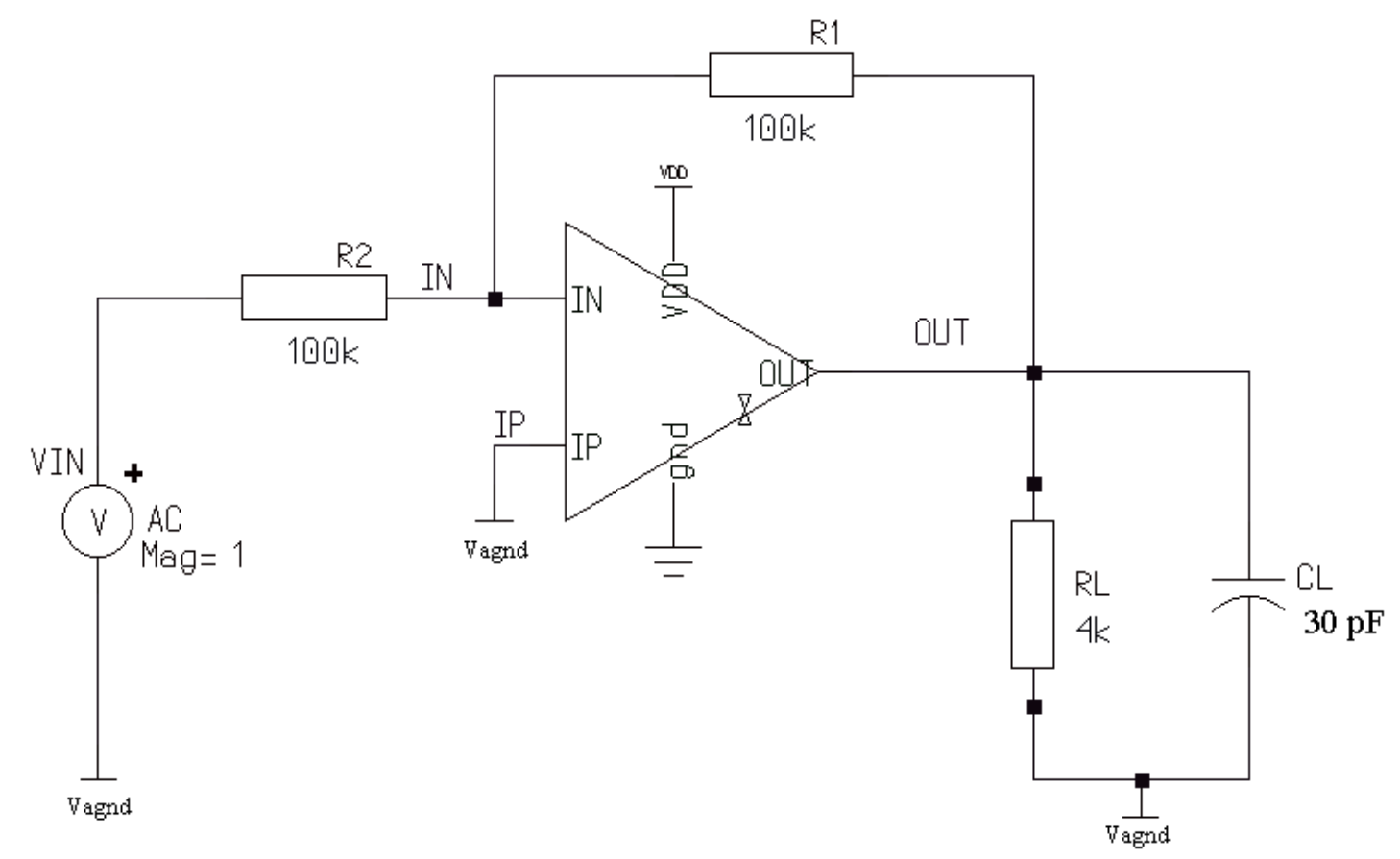

Figura 3.2. Testbench utilizado para a simulação do amplificador operacional folded cascode.

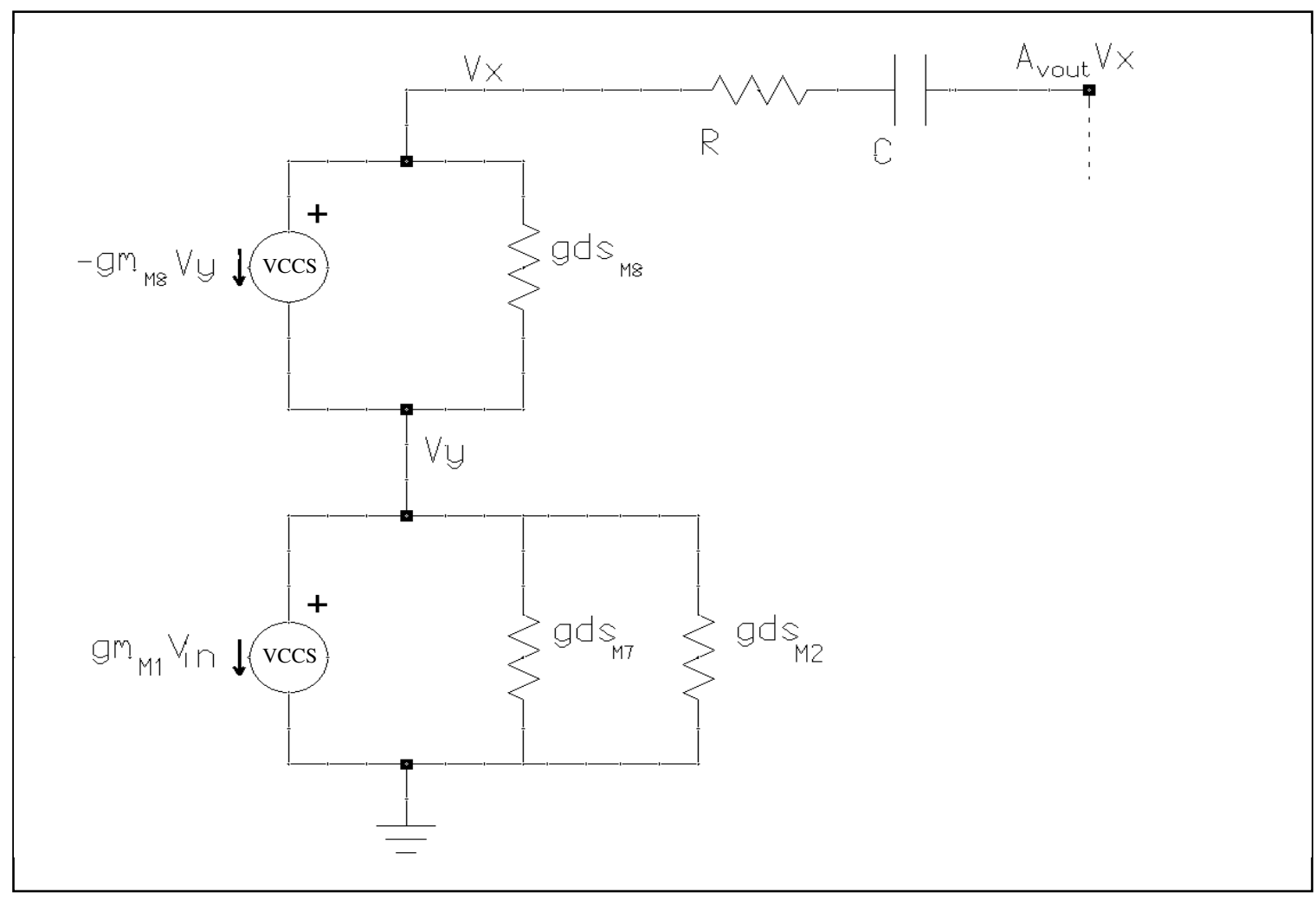

Figura 3.3. Circuito simplificado que modela o amplificador operacional folded cascode. 


$$
\left\{\begin{array}{l}
g m_{M 8} \cdot v_{y}=\left(v_{x}-v_{y}\right) \cdot g d s_{M 8}+\frac{v_{x} \cdot\left(A_{V_{\text {OUT }}}+1\right)}{R+\frac{1}{S \cdot C}} \\
\left(g d s_{M 7}+g d s_{M 2}\right) \cdot v_{y}+g m_{M 1} \cdot v_{I N}=-g m_{M 8} \cdot v_{y}+\left(v_{x}-v_{y}\right) \cdot g d s_{M 8}
\end{array}\right.
$$

onde $v_{x}$ e $v_{y}$ são tensões intermediárias, $v_{I N}$ é o sinal aplicado na entrada do amplificador, e

$A_{V_{\text {OUT }}}$ é o ganho do estágio de saída do amplificador;

$R=\frac{R_{C}}{2}$, onde $\mathrm{R}_{\mathrm{C}}$ é a resistência de compensação;

$C=2 \cdot C_{C}$, onde $\mathrm{C}_{C}$ é a capacitância de compensação;

Resolvendo o sistema de equações (3.1) e (3.2), em $v_{x}$, teremos,

$$
v_{x}=-\frac{g m_{M 1} \cdot v_{I N} \cdot\left(g m_{M 8}+g d s_{M 8}\right) \cdot\left(s \cdot R_{C} \cdot C_{C}+1\right)}{s \cdot\left(2 \cdot C_{C}\right) \cdot\left[\left(A_{V_{\text {OUT }}}+1\right) \cdot\left(g m_{M 8}+\left(g d s_{M 7}+g d s_{M 2}\right)+g d s_{M 8}\right)+\frac{R_{C}}{2} \cdot\left(g d s_{M 7}+g d s_{M 2}\right) \cdot g d s_{M 8}\right]}
$$

Pode-se notar que, dividindo os dois lados da equação anterior por $v_{\mathbb{N}} \mathrm{e}$ multiplicando por $A_{V_{\text {OUT }}}$, teremos,

$$
\begin{aligned}
& A_{V_{\text {OUT }}} \cdot \frac{v_{x}}{v_{I N}}= \\
& =A_{V}=-\frac{A_{V_{\text {OUT }}} \cdot g m_{M 1} \cdot\left(g m_{M 8}+g d s_{M 8}\right) \cdot\left(s \cdot R_{C} \cdot C_{C}+1\right)}{s \cdot\left(2 \cdot C_{C}\right) \cdot\left[\left(A_{V_{\text {OUT }}}+1\right) \cdot\left(g m_{M 8}+\left(g d s_{M 7}+g d s_{M 2}\right)+g d s_{M 8}\right)+\frac{R_{C}}{2} \cdot\left(g d s_{M 7}+g d s_{M 2}\right) \cdot g d s_{M 8}\right]}
\end{aligned}
$$

onde $A_{V}=$ ganho total do amplificador;

Para se obter a expressão de GBW, consideraremos que o zero introduzido pela compensação em frequência se encontra numa frequência muito elevada, conforme será mostrado na seção 3.2.3. Assim, substituindo $s=2 \pi \cdot f$, e considerando que na frequência $G B W$ o ganho total do circuito é unitário, $A_{V}=1$, vem,

$$
G B W=\frac{A_{V_{O U T}} \cdot g m_{M 1} \cdot\left(g m_{M 8}+g d s_{M 8}\right)}{2 \pi \cdot\left(2 C_{C}\right) \cdot\left[\left(A_{V_{\text {OUT }}}+1\right) \cdot\left(g m_{M 8}+\left(g d s_{M 7}+g d s_{M 2}\right)+g d s_{M 8}\right)+\frac{R_{C}}{2} \cdot\left(g d s_{M 7}+g d s_{M 2}\right) \cdot g d s_{M 8}\right]}
$$


Mais uma vez a expressão de GBW engloba os termos gds, que são inversamente proporcionais à tensão de Early, (2.24). Conforme dito na seção 2.1, há dificuldade em estimar, com precisão, o valor de gds. Por hora, o projeto será realizado de uma maneira onde não se faz necessário conhecer os valores de gds. O simulador será, por sua vez, utilizado como ferramenta efetiva de auxílio ao projeto.

Vamos, então, desprezar o termo ( $\left.g d s_{M 7}+g d s_{M 2}\right)$ em (3.3), supondo que ele seja muito menor que $\mathrm{gm}_{\mathrm{M} 8}$, o que é razoável na maior parte dos casos pois, normalmente, $g m>>g d s$. Com isto vários outros termos de (3.3) se cancelam, e a expressão (3.4) é obtida.

$$
G B W \approx \frac{A_{V_{O U T}} \cdot g m_{M 1}}{2 \cdot \pi \cdot\left(2 \cdot C_{C}\right) \cdot\left(A_{V_{\text {OUT }}}+1\right)} \approx \frac{g m_{M 1}}{2 \cdot \pi \cdot\left(2 \cdot C_{C}\right)}
$$

É importante ressaltar que, caso $\left(g d s_{M 7}+g d s_{M 2}\right)$ não seja desprezível frente à $g m_{M 8}$, essa simplificação acarreta um erro no cálculo da banda de ganho unitário. A expressão completa de GBW, (3.3), será aplicada para correção da banda a partir dos resultados obtidos com a simulação. Neste caso, os parâmetros $g d s_{M 7}, g d s_{M 2}$ e gds $s_{M 8}$ necessários serão extraídos do arquivo de saída da simulação.

\subsection{Descrição Funcional do ampOp Folded Cascode}

Uma descrição detalhada do funcionamento em pequenos e grandes sinais do amplificador folded cascode será apresentada aqui, de modo a facilitar o entendimento dos resultados de projeto.

\subsubsection{Análise de pequenos sinais}

A análise de pequenos sinais do circuito consiste em supor que os transistores estão polarizados com suas respectivas tensões e correntes $D C$ e que há uma pequena variação do sinal de entrada, suficiente para causar uma excursão do sinal na saída do circuito mas não suficiente para modificar o ponto de operação DC do circuito. Dessa forma, os parâmetros para pequenos sinais dos transistores, como a transcondutância gm, a condutância gds, etc., permanecerão aproximadamente constantes e, em consequência, também os parâmetros do amplificador que dependem destas grandezas, como o ganho $D C, G B W$, margem de fase, etc., serão mantidos constantes. Obviamente que, ao assumirmos que o ponto $D C$ de polarização não muda, estamos simplificando a análise. 
Uma vez que o amplificador esteja polarizado com as devidas tensões e correntes $D C$, vamos imaginar que um pequeno sinal $A C$ seja inserido em uma das entradas do circuito da figura 3.1, utilizando a topologia da figura 3.2. Dessa forma, como o ganho da topologia é unitário, o sinal de saída irá "seguir" o sinal da fonte de entrada com inversão de fase. Se observarmos as duas entradas do ampOp, a entrada positiva IP se encontra no potencial de "Terra $A C$ ", enquanto que a entrada negativa $I N$, devido ao enorme ganho do amplificador em malha aberta, tende a ter o mesmo potencial da entrada positiva, o que na literatura é chamado de curto virtual.

Porém, como o ganho de malha aberta não é infinito, a entrada IN apresentará um sinal muito pequeno que será amplificado pelo ampOp. Se esse sinal for maior que o sinal na entrada IP, pela figura 3.1 podemos verificar que o transistor $M 1$ começará a conduzir menos corrente, enquanto que o transistor M2 passará a conduzir mais corrente. Essa corrente (que pode ser considerada um sinal) será injetada no terminal de fonte do transistor $M 8$, o que tenderá a aumentar a tensão neste ponto. Como o terminal de porta de $M 8$ está polarizado com uma tensão constante, M8 tenderá a conduzir menos corrente. Porém, lembrando que estamos tratando de pequenos sinais, a fonte de corrente formada pelos transistores M11 e M12 continuará injetando uma corrente constante. Dessa forma, a corrente que M8 deixa de conduzir começa a carregar os capacitores de compensação, e a tensão no terminal de dreno de $M 8$ começará a subir. Logo, a tensão no terminal de porta do transistor $M N$ de saída começará a subir. Por se tratar de um sinal muito pequeno, essa excursão no terminal de porta de $M N$ também é pequena.

Vamos agora analisar o que acontecerá no terminal de porta do transistor de saída MP. Como a tensão no terminal de porta de $M N$, ou terminal de fonte de $M 15$, sobe, podemos verificar que a corrente que passa por M15 tende a diminuir, já que seu terminal de porta está polarizado com uma tensão $D C$ constante. Uma vez que $M 15$ passa a conduzir menos corrente, e a corrente injetada pela fonte formada por $M 11$ e M12 é constante, a corrente que deixa de ser conduzida por M15 começa a carregar o capacitor de compensação, aumentando a tensão no terminal de porta de $M P$, que é também o terminal de fonte de M16. Dessa forma, vemos que quando o terminal de porta de $M N$ sobe, o mesmo acontece para o terminal de porta de MP. Por essa razão, numa análise $A C$, consideramos que os terminais de porta de $M N$ e $M P$ estão curtocircuitados. Finalmente, analisando o sinal de saída, vemos que $M N$ passa a conduzir mais corrente enquanto que MP passa a conduzir menos corrente. Essa corrente extra 
que $M N$ conduz é fornecida pela resistência de carga, o que faz o sinal no terminal de saída baixar. Isso já era esperado, pois a pequena senóide de entrada, cuja derivada era positiva, estava aplicada no terminal de entrada negativa.

Analogamente à análise realizada para o caso acima, pode-se analisar o comportamento do circuito quando o sinal na entrada IN é menor do que a tensão na entrada $I P$.

\subsubsection{Análise de grandes sinais}

A análise de pequenos sinais realizada serve para termos uma idéia de como o circuito opera dinamicamente, e ajudar na decisão de como modificar seu projeto para atender algumas especificações.

A análise de grandes sinais vem para completar a análise de pequenos sinais, ajudando a entender o que ocorre quando um sinal muito grande é injetado na entrada do ampOp. Para realizar essa análise, vamos abrir a realimentação negativa da topologia da figura 3.2, e vamos injetar um degrau na entrada IN do ampOp, conforme indicado na figura 3.4.

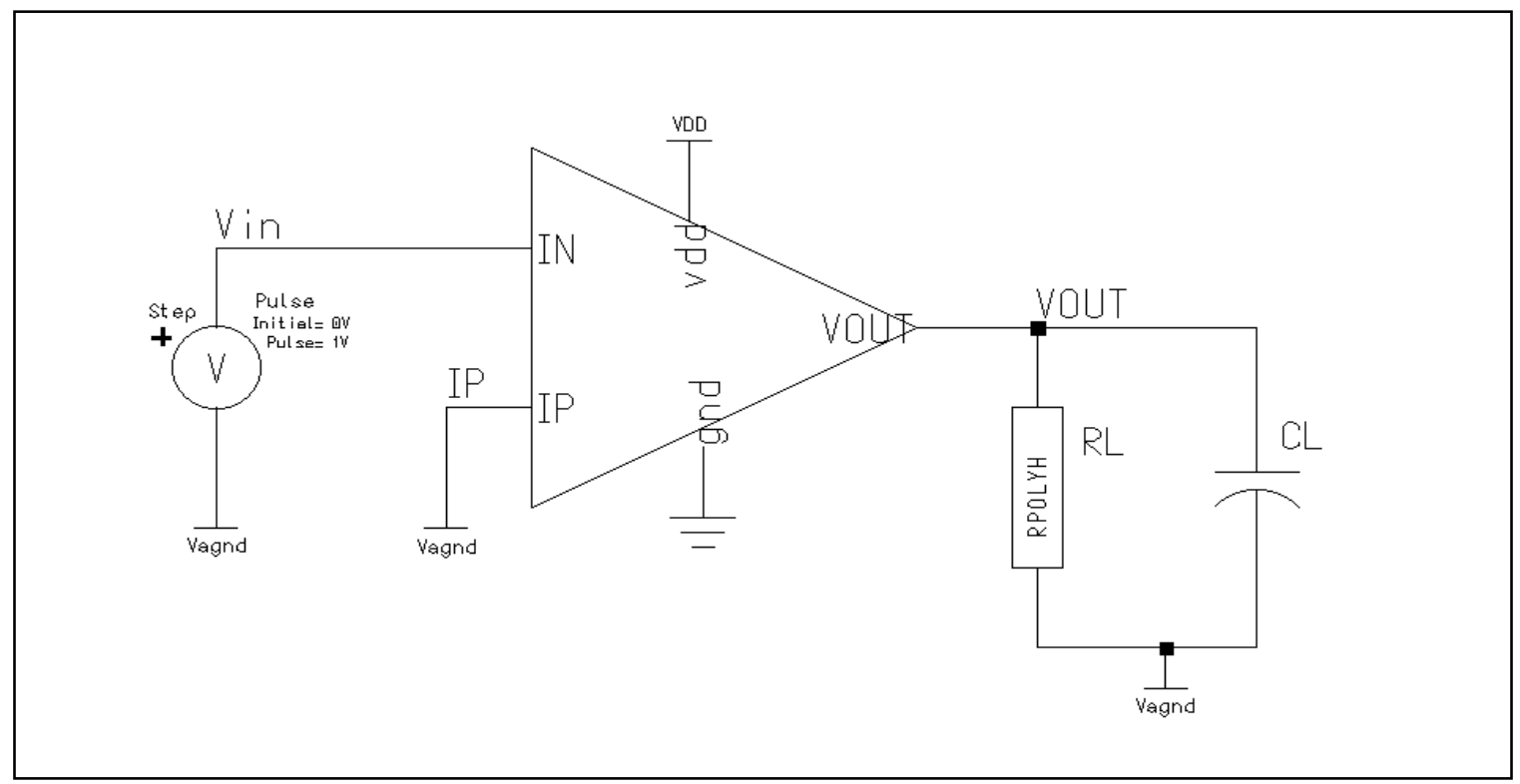

Figura 3.4. Testbench utilizado para simulação de grandes sinais.

Para essa análise, a amplitude do degrau será relativamente "grande" (por exemplo, $1 \mathrm{~V}$ pico-a-pico). O sinal de entrada e o sinal de saída são apresentados na figura 3.5 . 


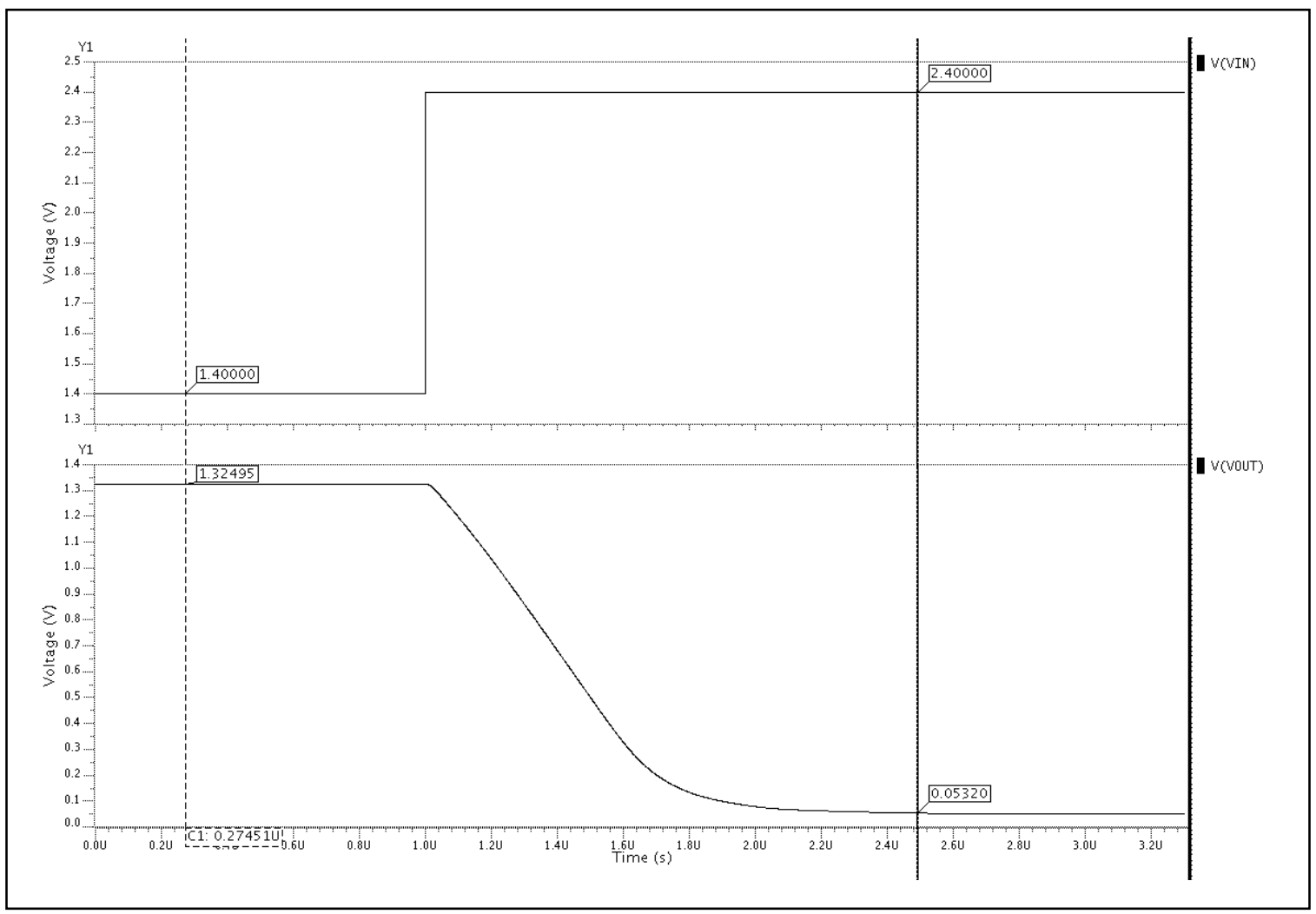

Figura 3.5. a) Degrau aplicado à entrada IN do ampOp e b) sinal na saída do ampOp.

Vamos iniciar a análise supondo que o sinal de entrada seja nulo. Ao mudar de $1,4 \mathrm{~V}, V_{\text {agnd }}$, para 2,4V , figura 3.5-a, toda a corrente de polarização do par diferencial de entrada passa a ser conduzida pelo transistor $M 2$, e o transistor $M 1$ é cortado. A corrente conduzida por $M 2$ é injetada no terminal de fonte de $M 8$, o que faz o potencial nesse ponto subir, tirando $M 2$ da saturação e cortando $M 8$, uma vez que seu terminal de porta está polarizado com uma tensão constante, figura 3.7-a. Isso faz com que o potencial no terminal de fonte de $M 2$ suba e, como a fonte de corrente formada por M3M4 não é ideal, a corrente injetada para a polarização do par diferencial irá diminuir, conforme ilustrado na figura 3.6-a, onde a corrente inicial era $I_{D}=12 \mu \mathrm{A}$ e após o degrau passa a $\operatorname{ser} I_{D}=3 \mu A$.

No outro ramo do folded, a corrente que $M 1$ deixa de injetar no terminal de fonte de M6 faz com que o potencial neste ponto caia. Como a corrente conduzida por M6 é constante, o potencial no seu terminal de dreno deve cair, tirando M6 da saturação e mantendo constante a corrente que ele conduz. Como o terminal de dreno de M6 polariza os terminais de porta de $M 5$ e $M 7$, a corrente conduzida por estes transistores deve diminuir. A corrente conduzida por M5 passa a ser a mesma que é conduzida por 
$M 6$, enquanto que no caso de $M 7$, apesar de sua tensão $V_{G S}$ ser a mesma de $M 5$, sua tensão $V_{D S}$ subiu o suficiente para que ele conduza a corrente injetada por $M 2$, uma vez que $M 8$ está cortado.

Como M8 está cortado, toda a corrente injetada no ramo pela fonte formada por M11-M12 passará a carregar o capacitor de compensação conectado ao terminal de porta de $M N$ (através do resistor de compensação).

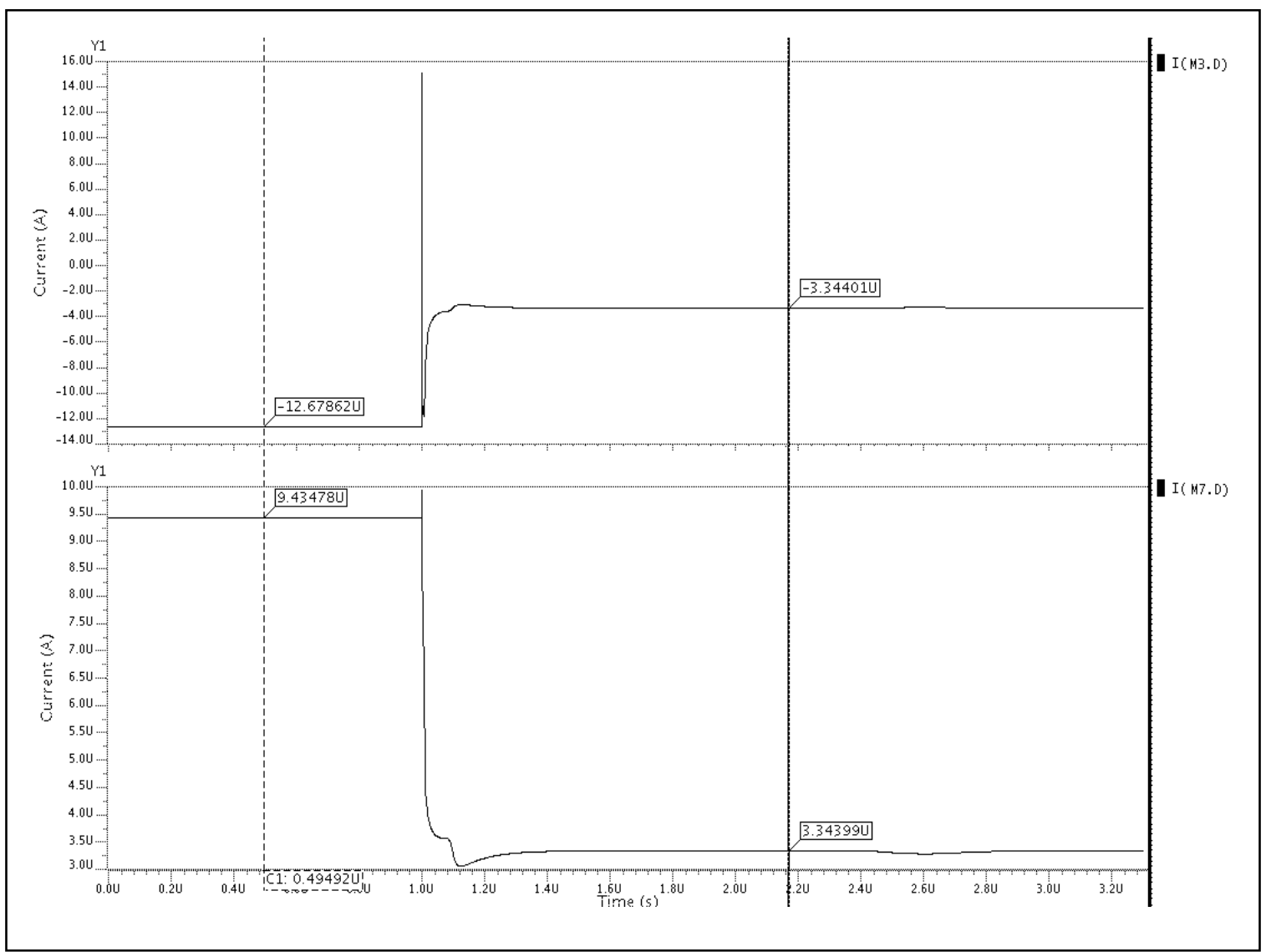

Figura 3.6. a) Corrente injetada pela fonte $M 3-M 4$ que polariza o par diferencial de entrada b) Corrente de dreno do transistor M7.

Assim, a tensão no terminal de fonte de M15 começa a subir, figura 3.7-b, e o transistor começa a cortar. A corrente que M15 deixa de conduzir passa a carregar o capacitor de compensação conectado ao terminal de porta de MP, figura 3.7-c. M16 também irá cortar, pois o potencial de seu terminal de dreno (fonte de M15) subirá, chegando à VDD. Como o capacitor conectado à fonte de M16 está sendo carregado, aumentando o potencial neste ponto, a fonte M11-M12 tenderá a ser cortada, pois sua tensão $V_{S D}$, entre os terminais fonte-dreno, diminuirá, diminuindo sua corrente, figura 
3.7-d. Ao final deste processo, os capacitores de polarização estarão carregados, não haverá corrente fluindo pelo ramo (com excessão do transistor $M 7$ que conduzirá toda a corrente de polarização do par diferencial injetada pelo transistor M2, figura 3.6-b), pois a fonte M11-M12 estará cortada, uma vez que a tensão no terminal de porta de $M P$ chega à $V D D$. Na saída, como $M N$ está conduzindo corrente máxima e $M P$ está praticamente cortado, toda a corrente solicitada por $M N$ é fornecida pela carga, e o sinal de tensão na saída será mínimo, ou seja, o inverso do sinal de entrada, conforme ilustrado na figura 3.5-b.

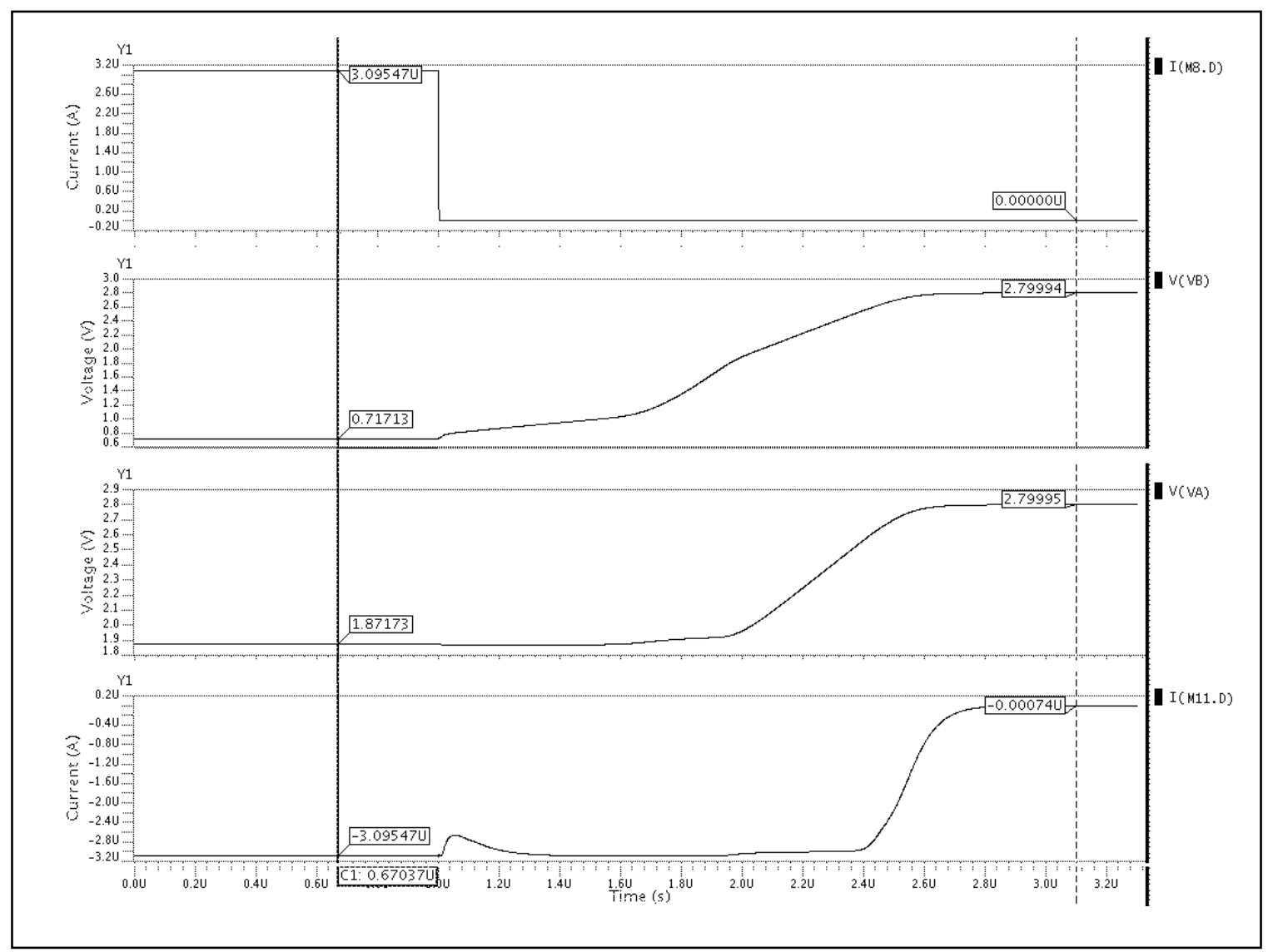

Figura 3.7. a) Corrente de dreno de M8. b) Tensão no terminal de fonte de M15 (terminal de porta de $M N$ ). c) Tensão no terminal de fonte de $M 16$ (terminal de porta de $M P$ ). d) Corrente injetada pela fonte formada por M11-M12. 


\subsection{Projeto}

Nesta seção será descrito o projeto do amplificador operacional folded cascode. Ele foi realizado de uma forma sequêncial, visando a construção posterior de um software de projeto.

Primeiramente será projetado o estágio de saída, utilizando como ponto de partida as especificações de GBW e margem de fase. Dessa forma é possível determinar a transcondutância desse estágio, dada por $g m_{O}=g m_{M P}+g m_{M N}$, onde $g m_{M P}$ e $g_{M N}$ representam as transcondutâncias dos transistores de saída, MP e $M N$.

Uma restrição adotada é que $g m_{M P}=g m_{M N}$ pois assim as tensões nos terminais de porta de $M P$ e $M N$ terão, aproximadamente, a mesma excursão.

\subsubsection{Projeto dos transistores MN e MP do estágio de saída}

Admitindo que o amplificador tem apenas dois pólos importantes, $f p_{1}<f p_{2}$, e considerando que os demais pólos estão numa frequência muito maior do que $f p_{2}$, temos então, ao substituir os valores de GBW e MF da tabela 3.1, na expressão (2.22),

$$
\begin{gathered}
\Phi_{M}=\arctan \left(\frac{f p_{2}}{G B W}\right) \Rightarrow 60^{\circ}=\arctan \left(\frac{f p_{2}}{5 M H z}\right) \Rightarrow f p_{2}=1,732 \cdot G B W \\
f p_{2}=8,66 \mathrm{MHz}
\end{gathered}
$$

Na seção 3.2.3 é explicado o posicionamento dos pólos $f p_{1}$ e $f p_{2}$ antes e depois da compensação em frequência. Utilizando o resultado (3.10) e substituindo o valor calculado de $f p_{2}$, podemos determinar $g m_{O}$,

$$
\begin{gathered}
f p_{2}=\frac{g m_{O}}{2 \cdot \pi \cdot C_{L}} \Rightarrow g m_{o}=2 \cdot \pi \cdot 8,66 \mathrm{MHz} \cdot 30 p F \\
g m_{o}=1,63 \mathrm{mS}
\end{gathered}
$$

Como

$$
g m_{O}=g m_{M P}+g m_{M N} \quad e \quad g m_{M P}=g m_{M N}
$$

então,

$$
g m_{M P}=g m_{M N}=\frac{g m_{O}}{2}=816,2 \mu S
$$

A corrente quiescente do estágio de saída deve ser escolhida levando-se em conta alguma especificação de consumo e o trade-off com a área dos transistores. Para 
o nosso caso, como não temos uma especificação de consumo, vamos escolher uma corrente de forma que a área dos transistores de saída não seja muito grande. Após algumas considerações, uma escolha razoável foi $I_{Q}=80 \mu A$. Supondo condição de inversão forte dos transistores, de (2.14) teremos,

$$
\begin{gathered}
g m_{M P}=\sqrt{2 \cdot I_{D} \cdot K_{P} \cdot\left(\frac{W}{L}\right)_{M P}} \Rightarrow 816,2 \mu=\sqrt{2 \cdot 80 \mu \cdot 58 \mu \cdot\left(\frac{W}{L}\right)_{M P}} \\
\left(\frac{W}{L}\right)_{M P}=\frac{85,3}{1}[\mu \mathrm{m} / \mu \mathrm{m}]
\end{gathered}
$$

Como as transcondutâncias dos transistores PMOS e NMOS de saída são iguais, a relação entre suas dimensões deve ser, aproximadamente,

$$
\left(\frac{W}{L}\right)_{M P}=\left(\frac{K_{P}}{K_{N}}\right) \cdot\left(\frac{W}{L}\right)_{M N} \Rightarrow\left(\frac{W}{L}\right)_{M P}=\left(\frac{170 \mu}{58 \mu}\right) \cdot\left(\frac{W}{L}\right)_{M N}=2,93 \cdot\left(\frac{W}{L}\right)_{M N}
$$

Logo,

$$
\left(\frac{W}{L}\right)_{M N}=\frac{29,1}{1}[\mu m / \mu m]
$$

Uma vez projetados os transistores de saída, vamos calcular as tensões $V_{G S}$ de $M N$ e $M P$ pois, na sequência, elas serão utilizadas no projeto dos transistores $M 5, M 6$, M7 e $M 8$.

3.2.2. Cálculo das tensões $\mathrm{Vgs}_{M N}$ e $\mathrm{Vgs}_{M P}$ (quiescentes) nos terminais de porta dos transistores de saída

Utilizando a corrente quiescente $I_{Q}=80 \mu A$, e as relações $W / L$ calculadas na seção 3.2.1, teremos:

$$
\begin{gathered}
80 \mu=\frac{1}{2} \cdot 170 \mu \cdot \frac{29,1}{1} \cdot\left(V_{G S_{M N}}-0,5\right)^{2} \Rightarrow V_{G S_{M N}} \approx 0,68 \mathrm{~V} \\
V_{G_{M N}} \approx 0,68-0 \Rightarrow V_{G_{M N}} \approx 0,68 \mathrm{~V} \\
80 \mu=\frac{1}{2} \cdot 58 \mu \cdot \frac{85,3}{1} \cdot\left(V_{G S_{M P}}-0,7\right)^{2} \Rightarrow V_{G S_{M P}} \approx 0,88 \mathrm{~V} \\
V_{G_{M P}} \approx 2,80-0,88 \Rightarrow V_{G_{M P}} \approx 1,92 \mathrm{~V}
\end{gathered}
$$




\subsubsection{Projeto dos capacitores, $C_{C}$, e resistores, $\boldsymbol{R}_{C}$, de compensação}

O próximo passo é projetar os componentes utilizados na compensação em frequência do amplificador, $C_{C}$ e $R_{C}$. Para o projeto de $C_{C}$, será estimada a capacitância parasita, $C_{P}$, no nó 1 da figura 3.1, sendo que $C_{C}$ deve ser escolhido de tal forma que $C_{p}$ não influa na operação. Na sequência, o resistor de anulamento de zero, $R_{C}$, é projetado para colocar o zero introduzido pela compensação, $z_{C}$, no infinito, pois este zero se encontra, a princípio, no semiplano direito do plano complexo, o que pode vir a degradar a margem de fase [9].

Antes da compensação em frequência, as posições do primeiro e segundo pólos podem ser expressas por:

$$
\begin{gathered}
f p_{1}=\frac{1}{2 \cdot \pi \cdot R_{o 1} \cdot C_{p}} \\
f p_{2}=\frac{g d s_{M P}+g d s_{M N}+g_{L}}{2 \cdot \pi \cdot C_{L}}
\end{gathered}
$$

onde $R_{01}$ representa a impedância equivalente no nó 1, e $C_{P}$ representa a somatória das capacitâncias parasitas também no nó 1 da figura 3.1. Com relação ao segundo pólo, que ocorre no nó 2, os termos $g d s_{M P}$ e $g d s_{M N}$ representam as condutâncias dos transiistores $M P$ e $M N$, respectivamente, $g_{L}$ representa a condutância de carga, que é o inverso de $R_{L}$, e $C_{L}$ representa a capacitância de carga.

Após a compensação, as expressões dos pólos são dadas [8], aproximadamente, por,

$$
\begin{gathered}
f p_{1}=\frac{1}{2 \cdot \pi \cdot R_{e q} \cdot\left(A_{V_{\text {ouT }}} \cdot C+C_{P}\right)} \\
f p_{2}=\frac{g m_{O}}{2 \cdot \pi \cdot C_{L}}
\end{gathered}
$$

sendo $f p_{1}$ o pólo dominante, $f p_{2}$ o pólo não-dominante e $C=2 \cdot C_{C}$. A escolha do capacitor de compensação deve ser feita de tal forma que,

$$
A_{V_{\text {OUT }}} \cdot C>>C_{P}
$$

para que a posição do primeiro pólo não dependa de componentes parasitas. Também se encontra na literatura [11] que o capacitor de compensação é, usualmente, escolhido como sendo igual à capacitância de carga, $C_{L}$. Neste caso teríamos,

$$
C \approx C_{L}=30 p F
$$


Este valor para o capacitor de compensação acarretaria a utilização de àrea excessiva do chip, e não adotaremos este caminho.

Para determinar o valor da capacitância de compensação vamos estimar o valor de $C_{P}$. Esta capacitância parasita é formada principalmente por $C_{G S}$ dos transistores $M N$ e MP. Para os transistores de saída trabalhando na saturação, de (2.7) teremos:

$$
\begin{aligned}
& C_{G S_{M N}} \approx \frac{2}{3} \cdot C_{O X}^{\prime} \cdot W \cdot L=\frac{2}{3} \cdot 4,6 f F \cdot 29,1 \cdot 1 \Rightarrow C_{G S_{M N}} \approx 90 \mathrm{fF} \\
& C_{G S_{M P}} \approx \frac{2}{3} \cdot C_{O X}^{\prime} \cdot W \cdot L=\frac{2}{3} \cdot 4,6 f F \cdot 85,3 \cdot 1 \Rightarrow C_{G S_{M P}} \approx 260 \mathrm{fF}
\end{aligned}
$$

onde $C_{O X}^{\prime}=4,6\left[f F / \mu m^{2}\right]$. Por fim, chegamos ao valor de $C_{P}$, que é a soma de (3.12) e (3.13):

$$
C_{P} \approx C_{G S_{M N}}+C_{G S_{M P}}=350 \mathrm{fF}
$$

Podemos aplicar a relação (3.11) para determinar o valor mínimo de capacitância de compensação. Para tanto devemos determinar o ganho do estágio de saída,

$$
A_{V_{\text {OUT }}} \approx g m_{O} \cdot R_{L}=1,63 m \cdot 4 k \Rightarrow A_{V_{\text {ouT }}} \approx 6,5\lfloor\mathrm{~V} / \mathrm{V}\rfloor
$$

substituindo (3.14) e (3.15) em (3.11) vem,

$$
\begin{gathered}
A_{V_{\text {VUT }}} \cdot C>>C_{P} \Rightarrow 6,5 \cdot C>>0,35 p F \\
C>>0,05 p F
\end{gathered}
$$

Como $C=2 \cdot C_{C}$, então $C_{C} \gg 0,025 \mathrm{pF}$.

Considerando que existem outras capacitâncias parasitas que não foram consideradas em $C_{P}$, uma escolha razoável para $C_{C}$, tanto em termos de área como de precisão com relação à posição do pólo, é

$$
C_{C}=0,75 p F
$$

que representa 30 vezes o valor estimado da capacitância parasita no nó 1.

O resistor da compensação em frequência utilizada, compensação Miller com resistor de anulamento de zero, é utilizado para compensar o zero introduzido por $C_{C}$, uma vez que ele aparece no semiplano direito do plano complexo [8], sendo dado pela expressão, 


$$
Z_{C}=\frac{1}{2 \cdot \pi \cdot C \cdot\left(\frac{1}{g m_{O}}-R\right)}
$$

onde $R=\frac{R_{C}}{2}$. Esse zero degrada a margem de fase, pois faz o ganho subir com 20 $\mathrm{dB} /$ dec e a fase cair $90^{\circ}$. O resistor de compensação $R_{C}$ deve ser projetado de forma a colocar este zero no infinito, ou seja, devemos garantir que,

$$
R=\frac{1}{g m_{O}} \Rightarrow R_{C}=\frac{2}{g m_{O}}
$$

Substituindo (3.5) em (3.16) podemos calcular facilmente o valor de $R_{C}$ :

$$
R_{C}=\frac{2}{1,63 m}=1,227 \mathrm{k} \Omega
$$

Na sequência, serão projetados os transistores do par diferencial de entrada. A expressão simplificada de GBW será utilizada para isso. Será, primeiramente, calculado o valor da transcondutância dos transistores $M 1$ e $M 2$. Depois os transistores serão projetados para trabalhar na região de inversão forte pois, nesta região, o ruído flicker gerado é menor do que nas regiões de inversão moderada ou inversão fraca [13]. Além disso, os valores de $W$ e $L$ serão dobrados, o que implica que a área do transistor é multiplicada por quatro, para, também, diminuir o ruído flicker.

Finalmente, será determinada a corrente de polarização $I_{\text {TAIL }}$ que irá polarizar os transistores $M 1$ e $M 2$.

\subsubsection{Projeto dos transistores de entrada M1 e M2}

Utilizando a expressão simplificada de GBW, (3.4), vamos substituir o valor calculado de $C_{C}$, e o valor especificado de $G B W$ :

$$
5 M H z=\frac{g m_{M 1}}{2 \cdot \pi \cdot(2 \cdot 0,75 p)} \Rightarrow g m_{M 1}=47 \mu S
$$

Em termos de ruído, transistores operando em inversão forte geram menos ruído flicker do que operando em inversão moderada ou fraca [13]. Logo, o par de entrada será projetado para operar na região de inversão forte. De acordo com (2.13),

$$
I_{D \lim }=K_{P} \cdot\left(\frac{W}{L}\right)_{M 1} \cdot 2 n \cdot U_{T}^{2} \Rightarrow I_{D \lim }=58 \mu \cdot\left(\frac{W}{L}\right)_{M 1} \cdot 2 \cdot\left(1+\frac{1}{58}\right) \cdot(27 m)^{2}
$$




$$
I_{D \lim }=0,114 \cdot\left(\frac{W}{L}\right)_{M 1} \mu A
$$

Substituindo (3.18) em (2.12), teremos,

$$
I_{D}>0,91 \cdot\left(\frac{W}{L}\right)_{M 1} \mu A
$$

Finalmente, substituindo (3.17) e (3.19) em (2.14) (utilizando a mínima corrente que já garanta o transistor em forte inversão),

$$
\begin{gathered}
g m_{M 1} \approx \sqrt{2 \cdot\left(0,91 \mu \cdot \frac{W}{L}\right) \cdot K_{P} \cdot \frac{W}{L}} \Rightarrow\left(\frac{W}{L}\right)_{M 1}=\frac{g m_{M 1}}{\sqrt{1,82 \mu \cdot K_{P}}}=\frac{47 \mu}{\sqrt{1 ., 82 \mu \cdot 58 \mu}} \\
\left(\frac{W}{L}\right)_{M 1, M 2}=\frac{4,6}{1}=\frac{9,2}{2}[\mu \mathrm{m} / \mu \mathrm{m}]
\end{gathered}
$$

Ao invés de se utilizar $L=1 \mu m$, será utilizado $L=2 \mu m$, e o valor de $W$ será dobrado, para que a relação $W / L$ se mantenha constante. Com isso, a área dos transistores de entrada foi multiplicada por quatro e, de acordo com (3.20), quanto maior esta área menor o ruído flicker causado por eles. Também, problemas com descasamento de transistores são reduzidos.

$$
\bar{v}^{2}=\frac{K_{f}}{W \cdot L \cdot \operatorname{Cox}} \cdot \frac{1}{f}
$$

onde,

$K_{f}$ é uma constante dependente da tecnologia [8], [9].

$\bar{v}^{2}$ é a densidade espectral de ruído flicker [8], [9].

O par diferencial de entrada é polarizado através de uma fonte de corrente, que fornece uma corrente $I_{\text {TAIL }}=m \cdot I_{B I A S}$, onde $m$ representa o fator de espelhamento. Assim, para cada transistor de entrada a corrente será $I_{D}=\frac{m \cdot I_{B I A S}}{2}$.

De acordo com (3.19), e considerando o pior caso de $I_{B I A S}$ da tabela 3.1, onde ocorre uma variação de $-20 \%$ no seu valor nominal, temos que garantir que os transistores estejam trabalhando em inversão forte. Assim, substituindo os valores de $I_{B I A S}-20 \%$ e $\left(\frac{W}{L}\right)_{M 1}$, e resolvendo para o fator $m$, 


$$
I_{D}=\frac{m \cdot I_{B I A S}}{2}>0,91 \cdot\left(\frac{W}{L}\right)_{M 1} \Rightarrow m>\frac{1,82 \mu}{1,2 \mu} \cdot \frac{9,2}{2}
$$

Logo, devemos ter um fator de espelhamento $m>6,93$. Escolhendo $m=8$, e considerando agora o caso nominal de $I_{B I A S}$

$$
I_{\text {TAIL }}=8 \cdot 1,5 \mu \Rightarrow I_{\text {TAIL }}=12 \mu \mathrm{A}
$$

Vamos dar início ao projeto dos ramos do amplificador folded cascode, escolhendo uma corrente de polarização, $I_{F O L D E D}$, para cada ramo. O projeto de $M 5$ e $M 7$ é feito rapidamente, o que não acontece para $M 6$ e $M 8$ pois estes sofrem de efeito de corpo. É escolhida então uma tensão de overdrive, diferença entre $V_{G S}$ e $V_{T H}$, para $M 6$ e M8 igual à tensão de overdrive de $M 5$ e $M 7$.

\subsubsection{Projeto de M5 e M7 / M6 e M8}

Primeiramente devemos calcular qual deve ser a corrente de polarização dos ramos do estágio folded cascode. Para isso, essa corrente deve ser projetada levando-se em consideração a especificação de Slew Rate (SR) do amplificador. Para este circuito, o nó responsável pelo Slew Rate é o nó 1, figura 3.1, e

$$
S R=\frac{I}{C} \approx \frac{I_{F O L D E D}}{2 \cdot C_{C}}
$$

Esta expressão é aproximada pois não foram consideradas as capacitâncias parasitas mas apenas a capacitância de compensação. Assim, utilizando o resultado da seção 3.2.3, onde foi calculada a capacitância de compensação, e o $S R$ da tabela 3.1 teremos, $I_{F O L D E D}=3 \mu A$.

Dessa forma, verifica-se que a corrente total nos transistores M5 e M7 será:

$$
I_{M 5, M 7}=\frac{I_{T A I L}}{2}+3 \mu=9 \mu A
$$

As dimensões de M5 e M7 podem ser determinadas substituindo (3.7) em (3.21), já que $V_{G S_{M 5}}=V_{G S_{M N}}$,

$$
\begin{gathered}
9 \mu=\frac{1}{2} \cdot 170 \mu \cdot\left(\frac{W}{L}\right)_{M 5} \cdot\left(V_{G S_{M 5}}-0,5\right)^{2}=\frac{1}{2} \cdot 170 \mu \cdot\left(\frac{W}{L}\right)_{M 5} \cdot(0,68-0,5)^{2} \\
\text { Como }\left(\frac{W}{L}\right)_{M 5}=\left(\frac{W}{L}\right)_{M 7}, \\
\left(\frac{W}{L}\right)_{M 5, M 7}=\frac{6,6}{2}[\mu \mathrm{m} / \mu \mathrm{m}]
\end{gathered}
$$


Para projetar M6 e M8, verifica-se que ambos os transistores sofrem de efeito de corpo, já que seus terminais de fonte não se encontram no mesmo potencial que seus terminais de corpo. Isso acarreta em mudanças na tensão de limiar, o que representa um problema, pois não temos como avaliar, de inicio, qual será seu valor já que não sabemos qual a tensão no terminal de fonte. Por essa razão, será adotada uma tensão de overdrive para M6 e M8, igual à tensão de overdrive dos transistores M5 e M7.

$$
V_{G S_{M 6}}-V_{T H_{M 6}}=V_{G S_{M 5}}-V_{T H_{M 5}}=0,18 \mathrm{~V}
$$

Logo, substituindo (3.22) em (3.23), vem,

$$
\begin{gathered}
3 \mu=\frac{1}{2} \cdot 170 \mu \cdot\left(\frac{W}{L}\right)_{M 6} \cdot\left(V_{G S_{M 16}}-V_{T H_{M 6}}\right)^{2}=\frac{1}{2} \cdot 170 \mu \cdot\left(\frac{W}{L}\right)_{M 6} \cdot(0,18)^{2} \\
\left(\frac{W}{L}\right)_{M 6, M 8}=\frac{2,2}{2}[\mu \mathrm{m} / \mu \mathrm{m}]
\end{gathered}
$$

A última parte compreende o projeto dos transistores M13, M14, M15 e M16. Os transistores $M 15$ e $M 16$ formam o circuito de controle da característica $A B$ do estágio de saída, enquanto que os transistores M13 e M14 são colocados no circuito para melhorar a simetria entre os dois ramos do amplificador, minimizando o offset sistemático (que se deve, basicamente, à assimetrias do circuito).

Para o projeto de $M 15$ (M13) e M16 (M14), serão consideradas duas situações distintas: a primeira é a situação quiescente de operação do circuito, onde não há sinal aplicado e as duas entradas do amplificador têm valor igual a $V_{\text {agnd, }}$ e os transistores de saída conduzem a corrente quiescente $I_{Q}=80 \mu A$; a segunda situação considera a condição em que há um sinal aplicado na entrada do circuito, e o sinal de saída se encontra ou no seu valor máximo ou no seu valor mínimo. Dessa maneira, um dos transistores de saída estará conduzindo a corrente máxima, enquanto que o outro transistor estará conduzindo uma corrente mínima.

A corrente mínima deve ser projetada de forma a manter o transistor que a conduz com uma tensão $V_{G S}$ ligeiramente superior à tensão de limiar $V_{T H}$. Dessa forma, quando o sinal estiver sendo chaveado entre os transistores de saída, teremos uma transição suave de condução entre ambos, evitando distorção cross-over [2], [8], [9]. 


\subsubsection{Projeto do circuito de controle AB, composto por M15 e M16}

De [2], vemos que uma escolha razoável para a corrente mínima é $I_{M I N}=\frac{I_{Q}}{2}$. Assim, $I_{M I N}=\frac{I_{Q}}{2}=40 \mu A$.

\section{Condição quiescente: não há sinal aplicado nas entradas do amplificador}

Na condição quiescente, vamos projetar M15 e M16 para que passe, por cada um deles, metade da corrente do ramo, ou seja, $\frac{I_{F O L D E D}}{2}=1,5 \mu A$. De acordo com (3.7), a tensão no terminal de fonte do transistor M15 é:

$$
V_{S_{M 15}}=V_{G S_{M N}}=0,68 \mathrm{~V}
$$

Verifica-se que M15 sofre de efeito de corpo, já que os potenciais dos terminais de substrato e fonte são diferentes. Vamos chamar sua tensão de limiar de $V_{T H N 1} \mathrm{e}$ assim, temos uma primeira equação:

$$
1,5 \mu=\frac{1}{2} \cdot 170 \mu \cdot\left(\frac{W}{L}\right)_{M 15} \cdot\left(V_{G_{M 15}}-0,68-V_{T H N 1}\right)^{2}
$$

\section{Condição de excursão de pico máximo do sinal de saída}

Nesta situação, o transistor de saída MP conduz a corrente máxima, composta pela corrente que vai para a carga $R_{L}$, somada à corrente mínima, $I_{M I N}=40 \mu A$, que vai para o transistor $M N$. A tensão no terminal de porta de $M N$ será:

$$
40 \mu=\frac{1}{2} \cdot 170 \mu \cdot \frac{29,1}{1} \cdot\left(V_{G S_{M I N}}-0,5\right)^{2} \Rightarrow V_{G S_{M I N}}=0,63 \mathrm{~V}
$$

Nesta condição, em que passa pelo transistor $M N$ a corrente mínima de $40 \mu A$, verifica-se que a corrente $I_{F O L D E D}$ passa quase totalmente pelo transistor M15. Além disso, vemos que o potencial no terminal de fonte de M15 mudou em relação ao caso quiescente e, por esse motivo, também a tensão de limiar deste transistor, que será chamada agora $V_{T H N 2}$. Lembrando que a tensão do terminal de porta de $M N$ é a tensão no terminal de fonte de $M 15$ (3.26), encontramos a segunda expressão:

$$
3 \mu=\frac{1}{2} \cdot 170 \mu \cdot\left(\frac{W}{L}\right)_{M 15} \cdot\left(V_{G_{M 15}}-0,63-V_{T H N 2}\right)^{2}
$$


Temos duas equações, (3.25) e (3.27), e quatro incógnitas: $V_{G_{M 15}},\left(\frac{W}{L}\right)_{M 15}$

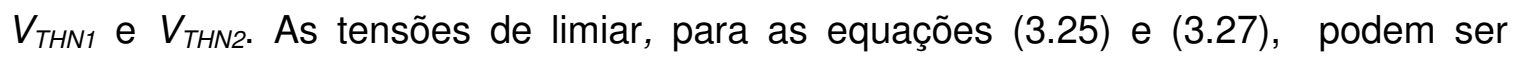
calculadas através de

$$
V_{T H}=V_{T H 0}+\gamma \cdot\left(\sqrt{2 \cdot\left|\phi_{F}\right|+V_{S B}}-\sqrt{2 \cdot\left|\phi_{F}\right|}\right)
$$

onde

$$
\gamma=\frac{\sqrt{2 \cdot \varepsilon_{S i} \cdot q \cdot N_{S U B}}}{C_{o x}} \quad \phi_{F}=\frac{k \cdot T}{q} \cdot \ln \left(\frac{N_{S U B}}{n_{i}}\right)
$$

$V_{T H N O}$ é a tensão de Limiar quando $V_{S B}=0$

$V_{S B}$ é a tensão entre corpo e fonte [V]

$2 \phi_{F}$ é o potencial da superfície em inversão forte;

$\gamma$ é um parâmetro de efeito de corpo;

$q$ é a carga do elétron;

$n_{i}$ é a concentração intrínseca de portadores no silício;

$\varepsilon_{S i}$ é permitividade do $\mathrm{Si}$;

$\frac{k \cdot T}{q} \approx 27 m V$, considerando a temperatura ambiente igual à $40^{\circ} \mathrm{C}$.

$N_{S U B}$ é a dopagem do substrato

- $\quad \mathrm{N}_{\mathrm{SUB}}(\mathrm{NMOS})=2,611 \mathrm{E} 17 \mathrm{At} / \mathrm{cm}^{3}$

- $\quad \mathrm{N}_{\text {SUB }}(\mathrm{PMOS})=9,24 \mathrm{E} 16 \mathrm{At} / \mathrm{cm}^{3}$

Tabela 3.2. Constantes Físicas.

\begin{tabular}{|c|c|c|c|}
\hline Constante & Descrição & Valor & Unidade \\
\hline$\varepsilon_{0}$ & Permitividade do vácuo & $8,854 \mathrm{E}-14$ & $\mathrm{~F} / \mathrm{cm}$ \\
\hline$\varepsilon_{\mathrm{Si}}$ & Permitividade do Si & $11 \varepsilon_{0}$ & $\mathrm{~F} / \mathrm{cm}$ \\
\hline $\mathrm{n}_{\mathrm{i}}$ & $\begin{array}{c}\text { Concentração intrínseca de } \\
\text { portadores }\left(40^{\circ} \mathrm{C}\right)\end{array}$ & $3,93 \mathrm{E} 10$ & $\mathrm{~cm}^{-3}$ \\
\hline
\end{tabular}


Substituindo os valores dados nas expressões em (3.29), teremos,

$$
\begin{gathered}
\phi_{F}=\frac{k \cdot T}{q} \cdot \ln \left(\frac{N_{S U B}}{n_{i}}\right)=0,43 \mathrm{~V} \\
\gamma=\frac{\sqrt{2 \cdot \varepsilon_{S i} \cdot q \cdot N_{S U B}}}{C_{O x}}=0,64 \mathrm{~V}^{1 / 2}
\end{gathered}
$$

Substituindo agora (3.24), (3.30) e (3.31) em (3.28), podemos calcular qual será a tensão de limiar $V_{T H N 1}$ para $M 15$, no caso quiescente:

$$
\begin{gathered}
V_{T H N 1}=0,5+0,64 \cdot(\sqrt{2 \cdot 0,43+0,68}-\sqrt{2 \cdot 0,43}) \\
V_{T H N 1}=0,7 V
\end{gathered}
$$

De (3.26), na condição de corrente mínima na saída, verificamos que a tensão entre os terminais de substrato e fonte do transistor M15 será,

$$
V_{S B_{M 15}}=V_{G S_{M N}}-0=0,63 \mathrm{~V}
$$

Substituindo (3.30), (3.31) e (3.33) em (3.28), podemos calcular qual será a tensão de limiar $V_{T H N 2}$ para $M 15$ :

$$
\begin{gathered}
V_{\text {THN } 2}=0,5+0,64 \cdot(\sqrt{2 \cdot 0,43+0,63}-\sqrt{2 \cdot 0,43}) \\
V_{\text {THN } 2}=0,69 \mathrm{~V}
\end{gathered}
$$

Substituindo os valores de $V_{T H N 1}$ (3.32) e $V_{T H N 2}$ (3.34) nas expressões (3.25) e (3.27), teremos duas expressões e duas incógnitas. Essas expressões são repetidas abaixo:

$$
\begin{aligned}
& \left\{\begin{array}{l}
1,5 \mu=\frac{1}{2} \cdot 170 \mu \cdot\left(\frac{W}{L}\right)_{M 15} \cdot\left(V_{G_{M 15}}-0,68-0,7\right)^{2} \\
3 \mu=\frac{1}{2} \cdot 170 \mu \cdot\left(\frac{W}{L}\right)_{M 15} \cdot\left(V_{G_{M 15}}-0,63-0,69\right)^{2}
\end{array}\right. \\
& \text { Resolvendo para }\left(\frac{W}{L}\right)_{M 15} \text {, } \\
& 3 \mu=\frac{1}{2} \cdot 170 \mu \cdot\left(\frac{W}{L}\right)_{M 15} \cdot\left(\sqrt{\frac{3 \mu}{170 \mu \cdot\left(\frac{W}{L}\right)_{M 15}}}+0,06\right)^{2}
\end{aligned}
$$




$$
\left(\frac{W}{L}\right)_{M 15}=\frac{1,7}{2}[\mu m / \mu m]
$$

O projeto do transistor PMOS, M16, será realizado utilizando-se a relação (3.6),

$$
\begin{gathered}
\left(\frac{W}{L}\right)_{M 16}=2,93 \cdot\left(\frac{W}{L}\right)_{M 15} \\
\left(\frac{W}{L}\right)_{M 16}=\frac{5}{2}[\mu \mathrm{m} / \mu \mathrm{m}]
\end{gathered}
$$

Finalmente, fazemos $M 13$ igual a $M 15$ e $M 14$ igual a $M 16$ :

$$
\left(\frac{W}{L}\right)_{M 13}=\frac{1,7}{2}[\mu \mathrm{m} / \mu \mathrm{m}] \quad\left(\frac{W}{L}\right)_{M 14}=\frac{5}{2}[\mu \mathrm{m} / \mu \mathrm{m}]
$$

\subsubsection{Projeto das fontes de corrente M3-M4, M9-M10 e M11-M12}

Para o espelho de corrente formado por MP1, MP2 e MP3, será escolhido o valor

$$
\left(\frac{W}{L}\right)_{M P 1}=\left(\frac{W}{L}\right)_{M P 2}=\frac{10}{2}[\mu \mathrm{m} / \mu \mathrm{m}] \text { e }\left(\frac{W}{L}\right)_{M P 3}=\frac{20}{2}[\mu \mathrm{m} / \mu \mathrm{m}]
$$

Para o transistor MP3 a relação $W / L$ foi dobrada, pois verificou-se que em um dos corners de projeto ele deixava a região de saturação e entrava na região linear de operação. Dessa forma, ao dobrarmos sua relação $W / L$, por (2.2) verifica-se que $V_{D S A T}$ diminui, o que nos dá uma margem maior para que MP3 continue na região de saturação.

De forma imediata, de acordo com o cálculo de $I_{\text {TAlL }}$ realizado na seção 3.2.4, teremos, ao considerar o caso nominal de $I_{B I A S}$ da tabela $3.1, \frac{I_{T A I L}}{I_{B I A S}}=8$ e portanto,

$$
\left(\frac{W}{L}\right)_{M 4}=8 \cdot\left(\frac{W}{L}\right)_{M P 3} \Rightarrow\left(\frac{W}{L}\right)_{M 3}=\left(\frac{W}{L}\right)_{M 4}=\frac{160}{2}[\mu \mathrm{m} / \mu \mathrm{m}]
$$

Observemos que é fixado que os transistores M3 e M4 têm as mesmas dimensões. Em geral é uma escolha razoável e facilita o projeto do circuito. 
Na seção 3.2.5 foi determinado $I_{F O L D E D}=3 \mu A$, que fornece $\frac{I_{F O L D E D}}{I_{B I A S}}=2$, logo,

$$
\left(\frac{W}{L}\right)_{M 12}=\left(\frac{W}{L}\right)_{M 10}=2 \cdot\left(\frac{W}{L}\right)_{M P 3} \Rightarrow\left(\frac{W}{L}\right)_{M 12}=\left(\frac{W}{L}\right)_{M 10}=\frac{40}{2}[\mu \mathrm{m} / \mu \mathrm{m}]
$$

Escolhemos ainda $M 10=M 9$ e $M 11=M 12$.

\subsubsection{Projeto do estágio de polarização}

Os transistores MP8, MP12, MP13, MP18 e MP19 são espelhos de corrente que repetem $I_{B I A S}$, e terão a mesma relação $W / L$ de $M P 3$. Para a tensão de polarização de M6 e M8, observemos que a seguinte equação deve valer se o transistor M5 estiver saturado:

$$
\left(V_{G S_{M 5}}-V_{T H_{M 5}}\right)+V_{G S_{M 6}}=V_{G S_{M P 11}}+V_{G S_{M P 10}}-V_{G S_{M P 9}}
$$

Escolhemos as dimensões dos transistores MP9, MP10 e MP11 para que $V_{G S M P 10} \approx V_{G S M 6}, V_{G S M P 11}=V_{G S M 5}$ e que $V_{G S M P 9} \approx V_{T H M 5}$. Primeiro MP8 é dimensionado para que a corrente que passa por MP10 e MP11 seja metade da corrente de M6 (ou um sexto da corrente de $\left.M 5\left(I_{D_{-} M 5}=I_{\text {TAIL }} / 2+I_{\text {FOLDED }}=6 \cdot I_{D_{-} M P 11}\right)\right)$. Assim, para que as tensões $V_{G S}$ de MP10 e MP11 sejam aproximadamente iguais às tensões $V_{G S}$ de $M 5 \mathrm{e}$ M6, devemos ter,

$$
\left(\frac{W}{L}\right)_{M P 10}=\left(\frac{W}{L}\right)_{M P 11}=\frac{1}{2}\left(\frac{W}{L}\right)_{M 6}=\frac{1}{6}\left(\frac{W}{L}\right)_{M 5}
$$

Facilmente se obtém ,

$$
\left(\frac{W}{L}\right)_{M P 10}=\left(\frac{W}{L}\right)_{M P 11}=\frac{1,1}{2}[\mu m / \mu m]
$$

Para que tensão $V_{G S}$ de $M P 9$ seja aproximadamente igual à tensão de limiar, MP9 deve ser projetado para trabalhar na região de inversão fraca. Assim, como a corrente que passa por MP9 é $1,5 \mu A$, de (2.12) devemos ter $I_{D}=1,5 \mu A<I_{D \lim } \mathrm{e}$ $\operatorname{LIM}<0,125$, de forma a garantir a operação na região de inversão fraca. Logo, $I_{D} \leq 0,125 \cdot I_{D \lim } \Rightarrow I_{D \lim } \geq 12 \mu \mathrm{A}$. Utilizando (2.12) e calculando $n=1,2$ [5], teremos, $\left(\frac{W}{L}\right)_{M P 9} \geq 40,3$. Vamos trabalhar com o valor de $L=0,35 \mu m$, para diminuir a área ocupada 
pelo transistor. Finalmente, será escolhido $\left(\frac{W}{L}\right)_{M P 9}=\frac{70}{0,35}[\mu \mathrm{m} / \mu \mathrm{m}]$, o que fornece um valor para o parâmetro $L I M=0,025$.

Determinemos agora as dimensões dos transistores MP4, MP5, MP6 e MP7. $M P 7$ espelha $I_{B I A S}$ e tem a mesma relação $W / L$ de $M P 1$ e $M P 2$, ou seja,

$$
\left(\frac{W}{L}\right)_{M P 1}=\left(\frac{W}{L}\right)_{M P 2}=\left(\frac{W}{L}\right)_{M P 7}=\frac{10}{2}[\mu \mathrm{m} / \mu \mathrm{m}]
$$

Estes transistores geram a tensão de polarização para os transistores cascode M3, M9, M11, MP13 e MP19. Vamos polarizá-los de forma a manter a tensão de dreno dos transistores $M 10, M 12, M P 12$ e MP18 o mais próximo de $V_{D D}$ e que ainda estejam saturados. Com isso aumentamos a excursão dos sinais. Nessas condições podemos escrever que:

$$
\left(V_{G S_{M 10}}-V_{T H_{M 10}}\right)+V_{G S_{M 9}}=V_{G S_{M P 4}}+V_{G S_{M P 5}}-V_{G S_{M P 6}}
$$

Escolhemos as dimensões dos transistores MP4, MP5 e MP6 para que $V_{\text {GSMP4 }}=$ $V_{G S M 10}, V_{G S M P 5} \approx V_{G S M 9}$ e que $V_{G S M P 6} \approx V_{T H M 10}$. Como a corrente de polarização que passa por MP5 e MP4 é metade da corrente que passa por M9 e M10, para que as tensões $V_{G S}$ de MP4 e MP5 sejam aproximadamente iguais às tensões $V_{G S}$ de $M 9 \mathrm{e}$ $M 10$, devemos ter,

$$
\left(\frac{W}{L}\right)_{M P 4}=\left(\frac{W}{L}\right)_{M P 5}=\frac{1}{2}\left(\frac{W}{L}\right)_{M 9}=\frac{1}{2}\left(\frac{W}{L}\right)_{M 10}
$$

obtendo-se,

$$
\left(\frac{W}{L}\right)_{M P 4}=\left(\frac{W}{L}\right)_{M P 5}=\frac{20}{2}[\mu m / \mu m]
$$

A tensão $V_{G S}$ de MP6 deve ser aproximadamente igual à tensão de limiar, $V_{T H}$, de $M 10$, e para isso MP6 deve ser projetado para trabalhar na região de inversão fraca. Assim, como a corrente que passa por MP6 é 1,5 $\mu A$, de (2.12) devemos ter $I_{D}=1,5 \mu A<I_{D \lim } \quad$ e $\quad L I M<0,125$. Logo, $I_{D} \leq 0,125 \cdot I_{D \lim } \Rightarrow I_{D \lim } \geq 12 \mu A$. Utilizando (2.12) e calculando $n=3,1$ [5], teremos $\left(\frac{W}{L}\right)_{M P 6} \geq 110,5$. Vamos trabalhar com o valor de $L=0,35 \mu m$, para diminuir a área ocupada pelo transistor. 
Finalmente, será escolhido $\left(\frac{W}{L}\right)_{M P 6}=\frac{120}{0,35}[\mu \mathrm{m} / \mu \mathrm{m}]$, o que fornece um valor para o parâmetro $L I M=0,033$.

Os transistores MP16 e MP17 são projetados para espelhar a corrente $I_{B I A S} \mathrm{e}$ têm a mesma relação $W / L$ de $M P 1$ e $M P 2$.

As tensões de polarização VGA e VGB, nos terminais de porta de M16 e M15, respectivamente, são geradas pelos transistores de polarização MP14 e MP15 (VGA), e MP20 e MP21 (VGB). A equação para $V G A$ é obtida através da malha formada pelos transistores $M P, M 16, M P 14$ e MP15, e pode ser expressa da seguinte maneira [5] [6],

$$
V_{G S_{M P}}+V_{G S_{M 16}}=V_{G S_{M P 14}}+V_{G S_{M P 15}}
$$

O circuito de polarização garante que

$$
V_{G S_{M 16}}=V_{G S_{M P 14}} \text { e } V_{G S_{M P}}=V_{G S_{M P 15}}
$$

Para garantir que $\mathrm{V}_{\mathrm{GS}}$ de $M 16$ e MP14 sejam iguais, os transistores e a corrente de dreno $I_{D}$ são iguais, então $M P 14=M 16$. O mesmo vale para $M P$ e $M P 15$.

$$
\left(\frac{W}{L}\right)_{M P 14}=\left(\frac{W}{L}\right)_{M 16}=\frac{5,2}{2}[\mu m / \mu m]
$$

para o caso de $M P 15$, na seção 3.2.1 o transistor de saída MP foi calculado como sendo, $\left(\frac{W}{L}\right)_{M P}=\frac{85,3}{1}[\mu m / \mu m]$. Para o layout do circuito os transistores de saída serão implementados como 60 transistores em paralelo, para minimizar o consumo de corrente do estágio de polarização, o que fornece uma relação $\left(\frac{W}{L}\right)_{\text {unitário_MP }}=\frac{\left(\frac{W}{L}\right)_{M P}}{60}=\frac{1,4216}{1}[\mu \mathrm{m} / \mu \mathrm{m}]$. Assim, por cada um dos transistores de saída unitários passará uma corrente de $\frac{I_{Q}}{60} \approx 1,3333 \mu \mathrm{A}$. Como pelo transistor MP15 passa uma corrente de $1,5 \mu \mathrm{A}$, a relação entre essas correntes será $\frac{1,5}{1,3333} \approx 1,1253$, que deve ser a mesma relação entre os transistores MP e MP15. Logo, 


$$
\left(\frac{W}{L}\right)_{M P 15}=1,1253 \cdot\left(\frac{W}{L}\right)_{\text {unitário_MP }}=\frac{1,6}{1}[\mu \mathrm{m} / \mu \mathrm{m}]
$$

Analogamente para a tensão $V G B$, a malha de polarização é formada pelos transistores $M N, M 15, M P 20$ e MP21 e a equação é dada por,

$$
V_{G S_{M N}}+V_{G S_{M 15}}=V_{G S_{M P 20}}+V_{G S_{M P 21}}
$$

Foram escolhidas,

$$
V_{G S_{M N}}=V_{G S_{M P 20}} \text { e } V_{G S_{M 15}}=V_{G S_{M P 21}}
$$

Como a tensão $V_{G S}$ de $M 15$ e $M P 21$ e a corrente de dreno $I_{D}$ são iguais, $M P 21=$ M15. O mesmo vale para MN e MP20.

$$
\left(\frac{W}{L}\right)_{M P 21}=\left(\frac{W}{L}\right)_{M 15}=\frac{1,7}{2}[\mu m / \mu m]
$$

para o caso de MP20, na seção 3.2.1 o transistor de saída $M N$ foi calculado como sendo $\left(\frac{W}{L}\right)_{M N}=\frac{29,1}{1}[\mu m / \mu m]$. O layout do transistor de saída $M N$ também será realizado como 60 transistores em paralelo, para minimizar o consumo de corrente do estágio de polarização, fornecendo uma relação $\left(\frac{W}{L}\right)_{\text {unitário_MN }}=\frac{\left(\frac{W}{L}\right)_{M N}}{60}=\frac{0,49}{1}[\mu \mathrm{m} / \mu \mathrm{m}]$. Assim, por cada um dos transistores de saída unitários passará uma corrente de $\frac{I_{Q}}{60} \approx 1,3333 \mu \mathrm{A}$. Como pelo transistor MP20 passa uma corrente de $1,5 \mu \mathrm{A}$, a relação entre essas correntes será $\frac{1,5}{1,3333} \approx 1,1253$, que deve ser a mesma relação entre os transistores MN e MP20. Logo,

$$
\left(\frac{W}{L}\right)_{M P 20}=1,1253 \cdot\left(\frac{W}{L}\right)_{\text {unitário_MN }}=\frac{0,55}{1}[\mu \mathrm{m} / \mu \mathrm{m}]
$$

Finalmente, a tabela 3.3 apresenta a relação $W / L$ de todos os transistores projetados para o amplificador folded cascode. 
Tabela 3.3. Transistores projetados para o ampOp folded cascode.

\begin{tabular}{|c|c|c|c|}
\hline TRANSISTOR & $\begin{array}{c}\text { Projeto } \\
\text { (W/L) }\end{array}$ & TRANSISTOR & $\begin{array}{l}\text { Projeto } \\
\text { (W/L) }\end{array}$ \\
\hline$M 1, M 2$ & $\frac{9,2}{2}$ & $\begin{array}{c}M P 1, M P 2 \\
M P 7\end{array}$ & $\frac{10}{2}$ \\
\hline$M 3, M 4$ & $\frac{160}{2}$ & $\begin{array}{l}M P 3, M P 4, \\
M P 5, M P 8\end{array}$ & $\frac{20}{2}$ \\
\hline$M 5, M 7$ & $\frac{6,6}{2}$ & MP6 & $\frac{120}{0,35}$ \\
\hline$M 6, M 8$ & $\frac{2,2}{2}$ & $M P 9$ & $\frac{70}{0,35}$ \\
\hline $\begin{array}{l}\text { M9, M10, } \\
M 11, M 12\end{array}$ & $\frac{40}{2}$ & $M P 10, M P 11$ & $\frac{1,1}{2}$ \\
\hline$M 13, M 15$ & $\frac{1,7}{2}$ & $M P 12, M P 13$ & $\frac{20}{2}$ \\
\hline$M 14, M 16$ & $\frac{5}{2}$ & MP14 & $\frac{5,2}{2}$ \\
\hline$M P$ & $\frac{85,3}{1}$ & $M P 15$ & $\frac{1,6}{1}$ \\
\hline \multirow[t]{4}{*}{$M N$} & $\frac{29,1}{1}$ & $M P 16, M P 17$ & $\frac{10}{2}$ \\
\hline & & MP18, MP19 & $\frac{20}{2}$ \\
\hline & & $M P 20$ & $\frac{0,55}{1}$ \\
\hline & & $M P 21$ & $\frac{1,7}{2}$ \\
\hline
\end{tabular}




\section{Capítulo 4}

\section{Software de Auxílio ao Projeto}

O software de auxílio ao projeto do amplificador operacional folded cascode foi escrito em linguagem de programação $C$, utilizando-se ao máximo a técnica de orientação à objetos. Nesta técnica o programa é dividido em pequenas partes e cada uma destas executa uma tarefa bem definida. A figura 4.1 apresenta o objeto amplificador folded cascode $(A O)$ subdividido em outros objetos menores, que são: estágio de entrada, estágio de polarização, estágio de saída, etc.
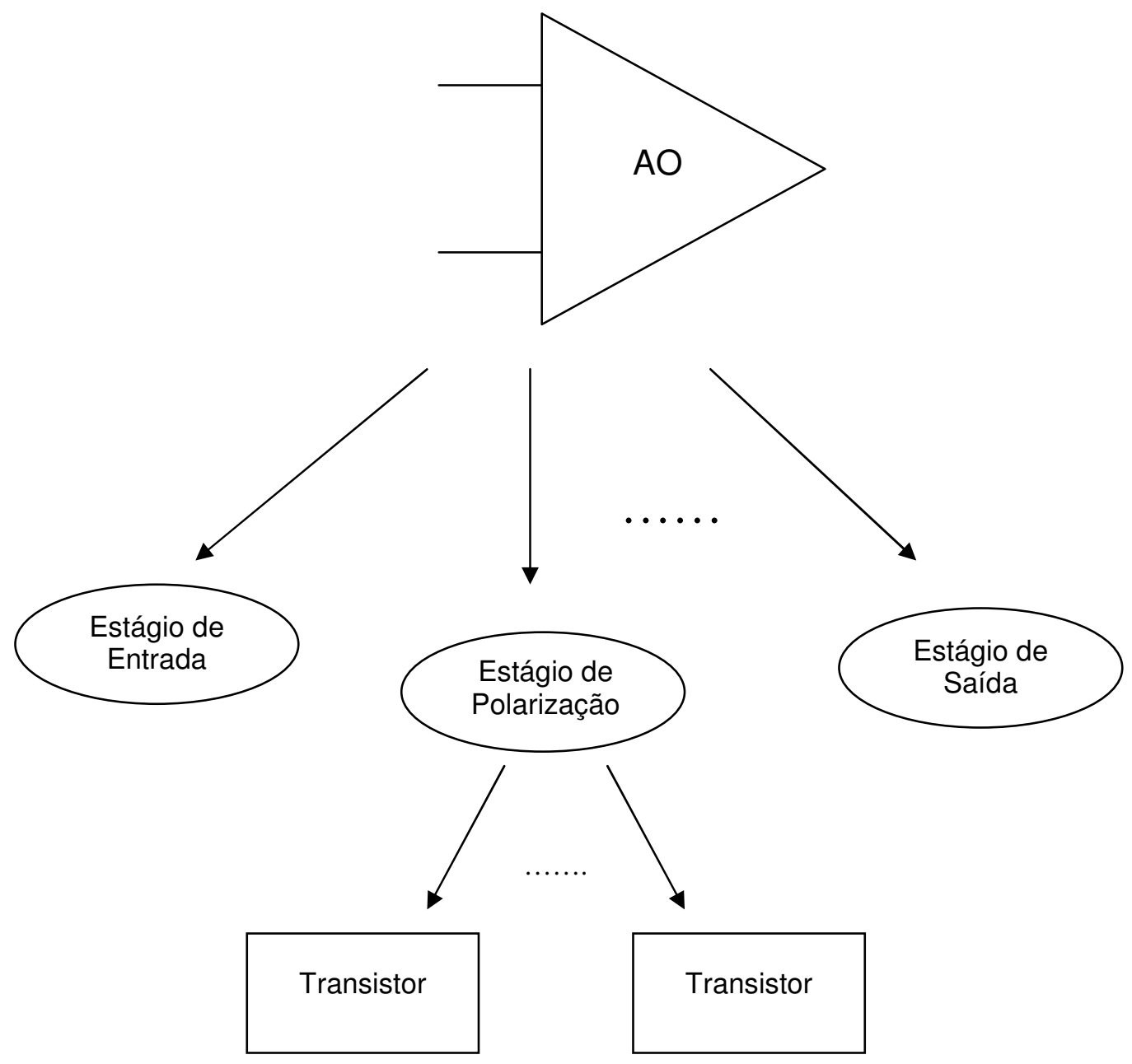

Figura 4.1. Objeto AO composto de outros objetos. 
Além disso, cada objeto que compõe o $A O$ também é composto de objetos menores, formados pelos transistores, resistores e capacitores. Essa estruturação do software, que segue as mesmas regras de estruturação de um circuito físico (hardware), permite que o software possa ser escrito como se estivéssemos montando um circuito numa protoboard. Esse é o grande benefício da programação orientada à objetos.

Para a implementação do software utilizando esta técnica, alguns tipos de variáveis foram criados. A figura 4.2 apresenta os objetos criados e a hierarquia entre eles.

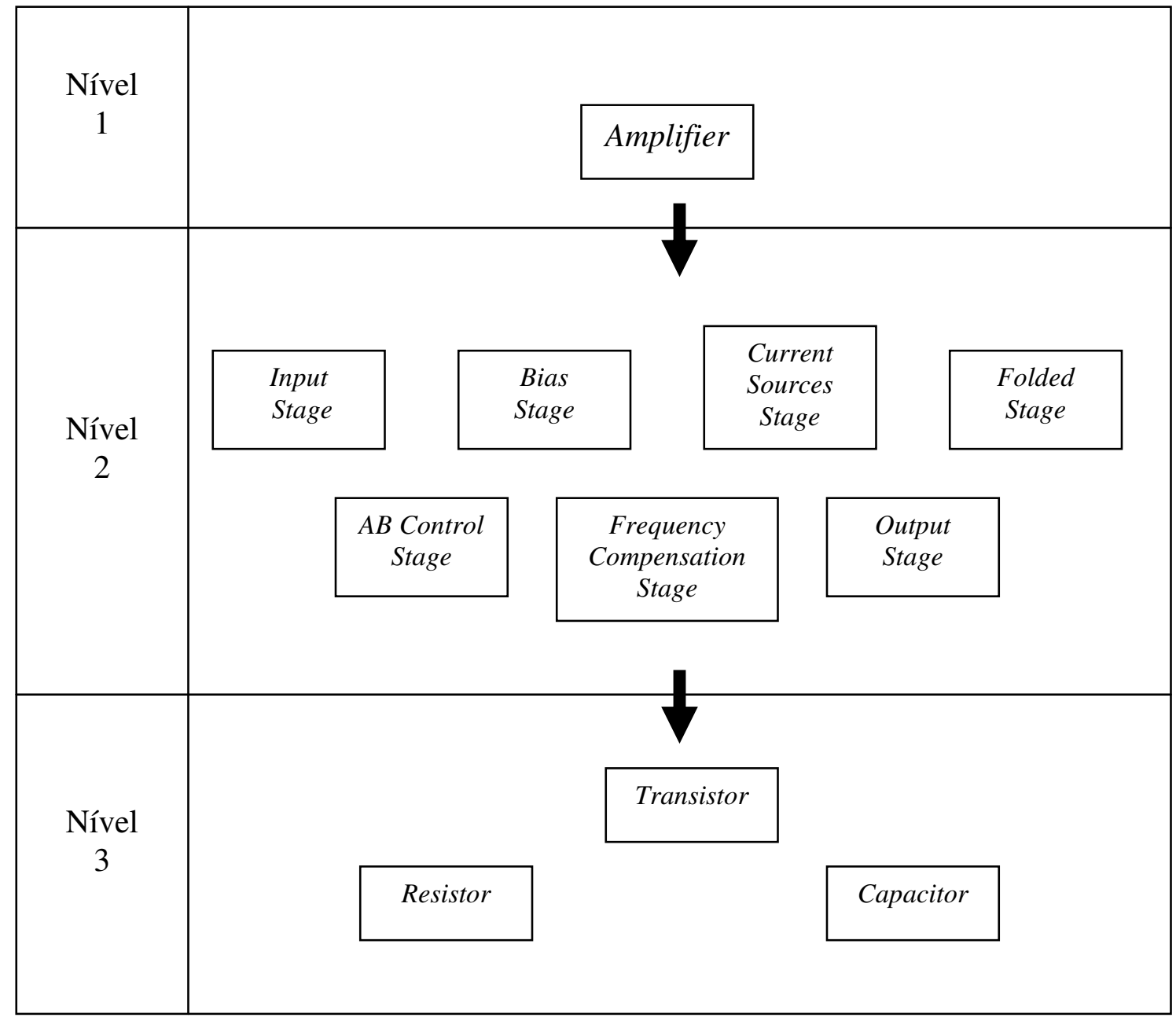

Figura 4.2. Hierarquia entre os objetos criados na linguagem $C$ para modelar o Amplificador Operacional Folded Cascode. 
O nível 1 representa a vista de mais alto nível do amplificador e é representado pelo objeto Amplifier. Cada objeto Amplifier é composto pelos objetos do nível 2 e, por sua vez, cada objeto do nível 2 é composto por objetos do nível 3.

Realizando o projeto do software da maneira proposta, será possível, futuramente, acrescentar novos objetos do tipo Amplifier que tenham uma topologia diferente da topologia Folded Cascode aqui estudada.

Para tornar o projeto do software ainda mais modularizado, cada objeto definido na figura 4.2 foi escrito como um arquivo “.c" próprio. Isso é ilustrado na tabela 4.1.

Tabela 4.1. Relação entre os objetos que formam o amplificador e os arquivos de código.

\begin{tabular}{|c|c|}
\hline Objeto & Arquivo de Código \\
\hline Bias Stage & biasstage.c \\
\hline Current Sources Stage & currentsourcesstage.c \\
\hline Input Stage & inputstage.c \\
\hline Folded Stage & foldedstage.c \\
\hline$A B$ Control Stage & abcontrolstage.c \\
\hline Frequency Compensation Stage & freqcompensationstage.c \\
\hline Output Stage & outputstage.c \\
\hline
\end{tabular}

Para a compilação do software, foi escrita uma rotina principal chamada design.c, que contém a rotina main, e um arquivo chamado Makefile utilizado para compilar todas as rotinas escritas na sequência correta. Para a correta compilação e utilização do software, é necessário ter instalados no computador uma versão do sistema operacional Linux com as bibliotecas de desenvolvimento para a linguagem $C$, além do compilador gcc e do programa make. Com o ambiente preparado para compilar este software, basta digitar o seguinte comando na linha de comando de uma shell: > make

O comando "make" gera um arquivo executável chamado "design". Adicionalmente, é necessário ter instalado o simulador elétrico Eldo, da empresa Mentor Graphics, pois o simulador será chamado para verificar alguns parâmetros dos transistores, e se as especificações fornecidas pelo projetista são atingidas. 


\subsection{Análise do Software de auxílio ao projeto}

A rotina principal, design.c, será analisada a seguir. Trechos dessa rotina serão reproduzidos no decorrer do texto, e a rotina completa pode ser encontrada no apêndice $A$ desta dissertação.

A figura 4.3 apresenta um trecho extraído da rotina design.c, que mostra a sequência utilizada para se realizar o projeto do amplificador operacional folded cascode.

1 * declara um amplificador */

struct Amplifier *folded_cascode;

/* Seta valores especificados para o amplificador */

readSpecification(folded_cascode);

/* Projeto do estágio de saída */

folded_cascode->output_stage->outputStageDesign(folded_cascode);

/* Projeto do estágio de compensação em freqüência */

folded_cascode->freqcompensation_stage->

->freqcompensationStageDesign(folded_cascode);

${ }^{*}$ Projeto do estágio de entrada */

folded_cascode->input_stage->inputStageDesign(folded_cascode);

$/{ }^{*}$ Projeto dos ramos do amplificador folded cascode */

folded_cascode->folded_stage->foldedStageDesign(folded_cascode);

/* Projeto do estágio de controle $\mathrm{AB}$ */

folded_cascode->abcontrol_stage->abcontrolStageDesign(folded_cascode);

/* Projeto das fontes de corrente do amplificador */

folded_cascode->currentsources_stage->

->currentsourcesStageDesign(folded_cascode);

/* Projeto do estágio de polarizacão */

folded_cascode->bias_stage->biasStageDesign(folded_cascode);

$/{ }^{*}$ Cria um arquivo .spi para simulacão */

writeSimulationFile(folded_cascode);

$/{ }^{*}$ Cria um arquivo .cir que descreve o tipo de análise a ser realizada *

writeAnalysisFile(folded_cascode);

/* Chama o simulador Eldo para uma simulacão AC do circuito projetado * system("eldo output.cir");

Figura 4.3. Trecho extraído da rotina design.c. 
Primeiramente um objeto do tipo Amplifier é declarado, sendo este objeto o ponteiro *folded_cascode. Este ponteiro é um endereço de memória que "aponta" para outro local de memória onde se encontra armazenada a estrutura Amplifier. A seguir, as especificações introduzidas pelo usuário são lidas e armazenadas no local de memória onde se encontra a estrutura Amplifier. Utilizando a sequência de projeto definida no capítulo 3, o projeto do amplificador é iniciado pelo estágio de saída, seguido pelo projeto do estágio de compensação em freqüência, e assim por diante. Após o término do primeiro projeto do amplificador, a função writeSimulationFile(folded_cascode) é chamada para escrever um arquivo netlist, contendo os componentes projetados e as suas ligações. A seguir, a função writeAnalysisFile(folded_cascode) é chamada para escrever um arquivo “.cir”, que contém o tipo de análise a ser realizada pelo simulador elétrico. Finalmente, a função system("eldo output.cir") passa para o sistema operacional a linha de comando "eldo output.cir" que inicia a simulação elétrica do circuito projetado. Depois da simulação ser concluida, a execução volta ao software de projeto.

Até este momento foi feito um primeiro projeto do amplificador operacional, utilizando-se equações simplificadas conforme explicado no capítulo 3. O próximo passo, após a simulação do circuito, é extrair os parâmetros de pequenos sinais que são relevantes para o nosso projeto, como por exemplo a condutância gds ou a transcondutância $g m$, e verificar se os resultados de simulação estão de acordo com as especificações do projeto. Isso é apresentado na figura 4.4 que mostra outro trecho extraído da rotina design.c.

/ Lê os parâmetros gds do arquivo de saída do simulador */

folded_cascode->gds_M7 = getGds("M7");

folded_cascode->gds_M8 = getGds("M8");

folded_cascode->gm_M1 = getGm("M1");

/* Calcula a banda GBW com os parâmetros extraídos do arquivo de saída */

folded_cascode->setGbwFull(folded_cascode);

/* Lê a Margem de Fase e GBW do Arquivo de Saída */

folded_cascode->phase_margin_sim = getPhaseMargin("PHASE_MARGIN");

folded_cascode->gbw_sim

= getPhaseMargin("GBW");

$/^{*}$ Verifica se há a necessidade de reprojetar o ampOp, e faz o reprojeto *

designCheck(folded_cascode);

Figura 4.4. Trecho extraído da rotina design.c. 
Logo após a simulação, os parâmetros de pequenos sinais podem ser lidos através da função getGds( ) ou getGm( ), que recebem como parâmetro uma string especificando o transistor a ser lido. A seguir, a função setGbwFull(folded_cascode) calcula a banda do circuito utilizando a expressão completa de GBW. Esse cálculo serve apenas para confirmar que a expressão completa é mais próxima da banda real do que a expressão simplificada (capítulo 3) pois, como temos o arquivo de resultados de simulação, a banda e a margem de fase do circuito são retirados diretamente desse arquivo, permitindo realizar um projeto com maior precisão. Esses parâmetros se encontram disponíveis no arquivo de saída da simulação "output.chi”, já que no arquivo que descreve a simulação a ser realizada, "simulation.cir", foi utilizado o comando .PRINT e especificado como parâmetros o ganho, em dB, e a fase, em graus, do amplificador. A margem de fase e a banda do circuito são lidos através das funções getPhaseMargin(“PHASE_MARGIN") e getPhaseMagin(“GBW”).

Finalmente, é chamada a função designCheck(folded_cascode), que irá verificar os resultados de simulação, comparar com a especificação, e reprojetar o amplificador, caso seja necessário. Serão feitos tantos reprojetos quanto necessários para que as especifiações sejam atendidas.

\subsubsection{Análise da rotina designCheck(Amplifier *thisAmplifier)}

A rotina designCheck recebe como parâmetro um ponteiro para um objeto Amplifier, que contém os resultados do projeto do amplificador, as especificações fornecidas pelo projetista e os resultados da simulação elétrica. A função primeiramente verifica se as especificações foram atendidas e, caso contrário, dará início às tentativas de reprojeto do amplificador até atendê-las. A figura 4.5 apresenta a máquina de estados finita que será utilizada para reprojetar o ampOp. Em cada estado, determinado pela figura do losango, é realizada uma chamada ao simulador elétrico, que realiza uma simulação $A C$ do amplificador. São retirados os parâmetros $G B W$ e $M F$ do arquivo de saída da simulação e eles são comparados às especificações. Dependendo do resultado da comparação, o software tomará uma decisão sobre qual caminho deverá seguir. As ações de reprojeto estão identificadas pela figura do retângulo. 


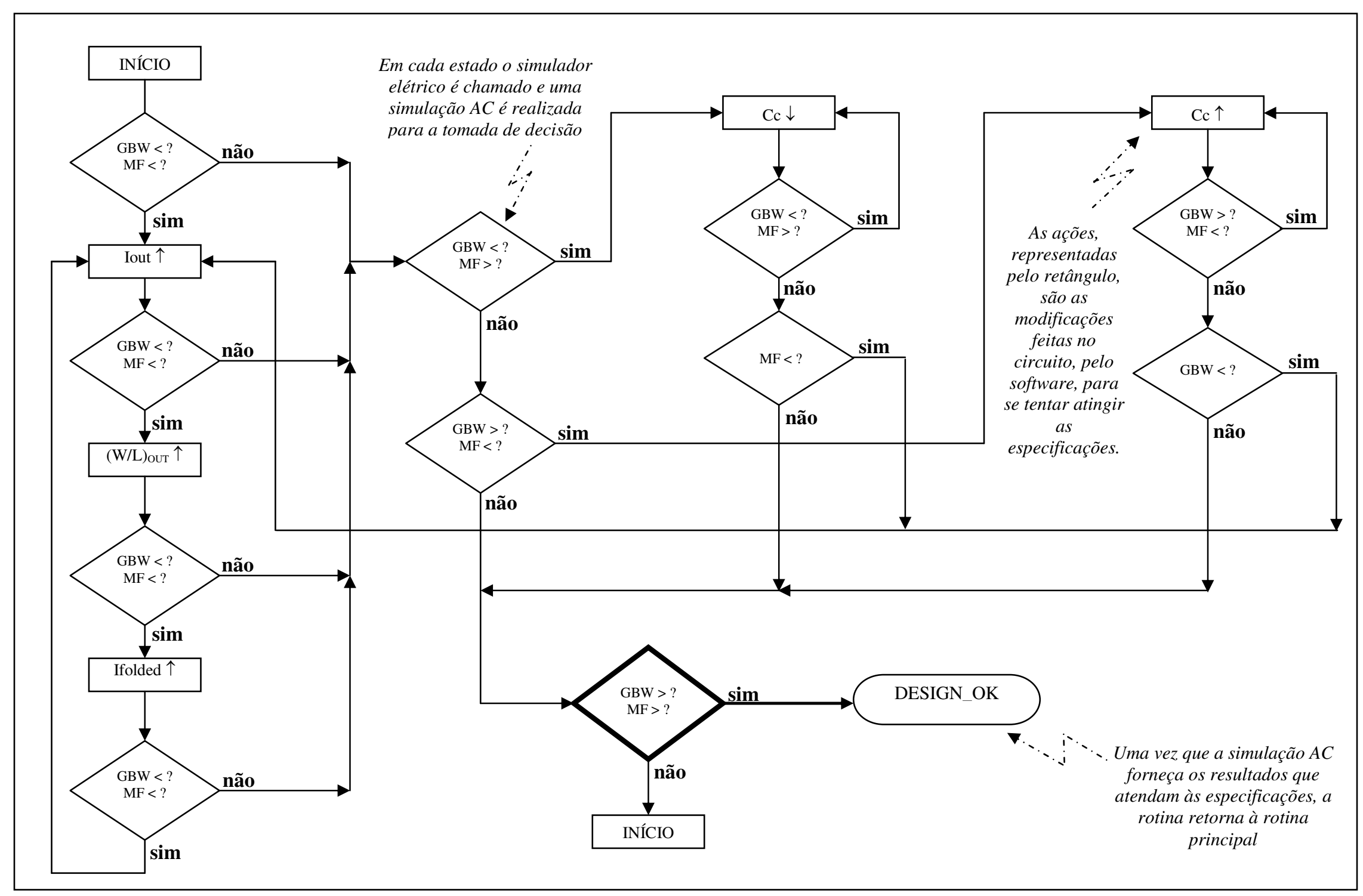

Figura 4.5. Diagrama de estados da rotina designCheck( ). 
Para realizar o reprojeto do amplificador, foram restringidas quais as características do circuito são alteradas e como são alteradas. Com isso desejamos manter o controle sobre o que estamos modificando e o que não queremos modificar no circuito. Como um exemplo do que não desejamos, imaginemos que $G B W$ obtido seja menor do que o especificado. Para aumentá-lo, poderíamos, entre outras opções, aumentar a corrente de polarização dos transistores do par diferencial de entrada. Isso acarretaria no aumento da transcondutância gm desses transistores, no aumento do ganho do circuito, e, por (2.17), no aumento esperado de GBW [8] [9]. Porém, ocorre que, ao aumentarmos a corrente de polarização dos transistores de entrada, estaríamos também aumentando a corrente de polarização dos transistores $M 5$ e M7, dos ramos do folded cascode. Ao aumentar sua corrente, seus parâmetros de pequenos sinais seriam modificados e, consequentemente, a posição de alguns dos pólos do circuito seria indesejadamente alterada.

Por essa razão, para uma primeira versão deste software, foram escolhidos apenas algumas características do circuito para serem trabalhadas. A tabela 4.2 apresenta estas características.

Tabela 4.2. Características escolhidas para serem modificados durante o reprojeto do amplificador.

\begin{tabular}{|c|c|c|}
\hline $\begin{array}{c}\text { Características } \\
\text { Modificadas }\end{array}$ & Modificação & Resultado \\
\hline IOUT & $\uparrow$ & GBW $\uparrow$, MF $\uparrow$ \\
\hline (W/L) OUT & $\uparrow$ & Equilibrar o ponto DC \\
\hline I $_{\text {FOLDED }}$ & $\uparrow$ & $\begin{array}{c}\text { Jogar pólos internos para } \\
\text { altas freqüências }\end{array}$ \\
\hline $\mathrm{C}_{\mathrm{C}}$ & $\uparrow$ & GBW $\downarrow, \mathrm{MF} \uparrow$ \\
\hline $\mathrm{C}_{\mathrm{C}}$ & $\downarrow$ & GBW $\uparrow, \mathrm{MF} \downarrow$ \\
\hline
\end{tabular}


A modificação da cada um das características apontadas na tabela 4.2 acarreta numa modificação controlada de algum parâmetro do circuito, sem modificar outros parâmetros. São elas:

- Iout: Ao se analisar a expressão completa de GBW (3.3), verifica-se que a banda de ganho unitário aumenta com o aumento do ganho do estágio de saída, $A v_{\text {OUT }}$, mantendo-se todos os outros parâmetros constantes. Sendo o ganho $A v_{\text {OUT }}$ diretamente proporcional à raiz quadrada da corrente de polarização do estágio de saída (3.15), IOUT, seu aumento acarreta num aumento de GBW. Além de aumentar GBW, ao se aumentar $I_{O U T}$, aumentase também a margem de fase do circuito (supondo que o amplificador tenha apenas dois pólos dominantes), pois de acordo com (3.10), ao se aumentar a transcondutância dos transistores de saída, $g m_{O}$, o segundo pólo é jogado para freqüências mais elevadas. Porém, como ocorre também o aumento de $G B W$, a margem de fase não aumenta tanto quanto aumentaria se $G B W$ fosse constante;

- $(W / L)_{\text {OUT }}$ : Em certas circunstâncias, ao se aumentar a corrente de saída sem aumentar o tamanho dos transistores, o ponto de operação $D C$ da saída sai do ponto médio entre as tensões de alimentação (resultado observado através de simulações). Dessa forma, para balancear novamente o circuito, a relação $(W / L)_{\text {OUT }}$ deve ser modificada;

- I I FOLDED: Algumas vezes, quando se deseja aumentar a margem de fase, não é suficiente jogar o segundo pólo para freqüências mais elevadas, pois outros pólos internos do amplificador começam a influenciar na resposta em freqüência do circuito. Para também deslocar estes outros pólos, a corrente de polarização dos ramos do folded cascode, I IFOLDED, é aumentada. Isso faz com que a impedância dos transistores diminua, já que estamos injetando mais corrente nos ramos. Ao diminuir a impedância, os pólos se movimentam para freqüências maiores. Deve-se atentar que isso eleva o Slew Rate do circuito;

- $\mathbf{C}_{\mathbf{c}}$ : A capacitância de compensação exerce influência direta sobre a banda e a margem de fase do circuito. Isso ocorre porque, devido ao efeito Miller, a capacitância que determina o primeiro pólo dependente fortemente deste capacitor. Logo, ao aumentarmos seu valor, o primeiro pólo se movimenta para freqüências mais baixas, diminuindo GBW mas aumentando a margem de fase. Por outro lado, ao diminuirmos $C_{C}$, o primeiro pólo se movimenta 
para freqüências mais elevadas, aumentando $G B W$ mas diminuindo a margem de fase.

Ao modificar esse conjunto de caracteristicas de forma organizada e sequêncial, podemos realizar o reprojeto do amplificador $e$ atender às especificações iniciais. Obviamente que outros conjuntos de características podem ser escolhidas quando se deseja reprojetar o amplificador; pode-se também reprojetar o amplificador como um todo, seguindo a sequência realizada para o primeiro projeto. Entretanto, ao se escolher esta última alternativa, o projetista terá menor controle sobre os parâmetros do circuito e pode ter dificuldades para atingir seus objetivos.

A elaboração do software foi realizada da seguinte maneira: inicialmente, ele foi escrito tendo-se como referência o primeiro projeto do ampOp, apresentado no capítulo 3. Os valores de $W / L$ dos transistores dados pelo software eram comparados com os valores do projeto de referência, até que fossem relativamente próximos. Uma vez concluída esta fase inicial, deu-se início à elaboração da rotina utilizada pelo software para reprojetar o ampOp (de forma a atender novas especificações), onde foi implementada a máquina de estados descrita na seção 4.1.1. Como forma de validar o reprojeto do amplificador, algumas especificações foram modificadas, como por exemplo $G B W=20 \mathrm{MHz}, M F=80^{\circ}$, etc., sendo que esses novos resultados foram validados através de simulações. 


\section{Capítulo 5}

\section{Resultados de Simulaç̃es}

Este capítulo tem por finalidade apresentar as simulações realizadas para verificação das características do amplificador projetado no capítulo 3. Após essa verificação, deu-se início à realização do layout e, posteriormente, à sua fabricação. Todas as simulações apresentadas correspondem ao caso típico, $40{ }^{\circ} \mathrm{C}, I_{B I A S}=$ $1,5 \mu A$. Porém, os corners que englobam os casos Worst Case Power e Worst Case Speed também foram simulados, considerando a faixa de temperatura $\left(-25^{\circ} \mathrm{C}, 40^{\circ} \mathrm{C}\right.$ e $120^{\circ} \mathrm{C}$ ) e a variação de $I_{B I A S}$ especificada na tabela 3.1.

\subsection{Input Common Mode Range - ICMR}

O parâmetro ICMR objetiva identificar os limites de operação do amplificador com relação à tensão de porta dos transistores do par diferencial de entrada (tensão de modo comum). O testbench da figura 5.1 foi utilizado para realizar essa simulação.

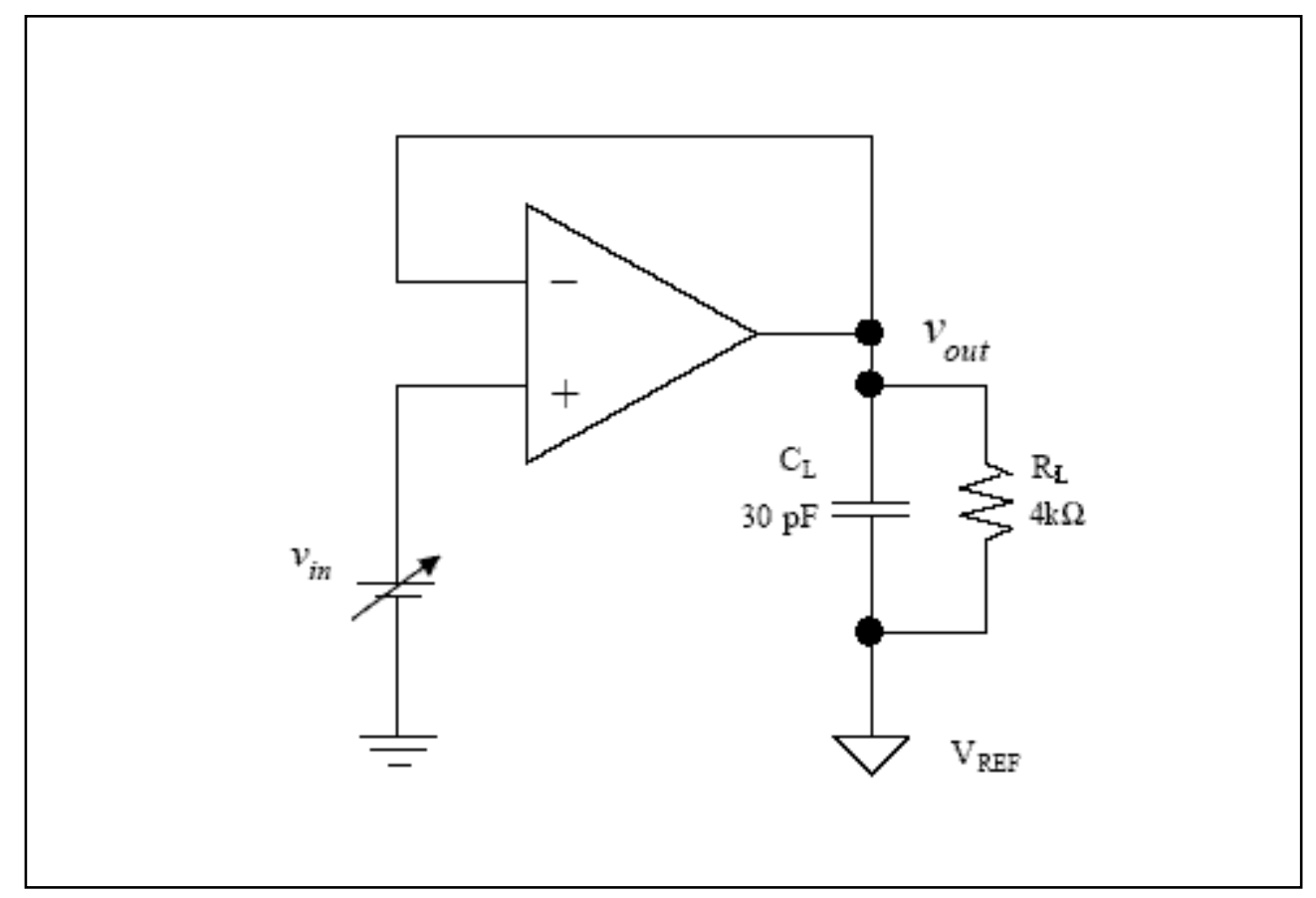

Figura 5.1. Testbench utilizado para simulação e medida de ICMR.

Foi realizada uma simulação $D C$, com variação da tensão da fonte $V_{\text {in }}$ entre [1 ; 3] $V$, e o resultado dessa simulação é apresentado na figura 5.2. 


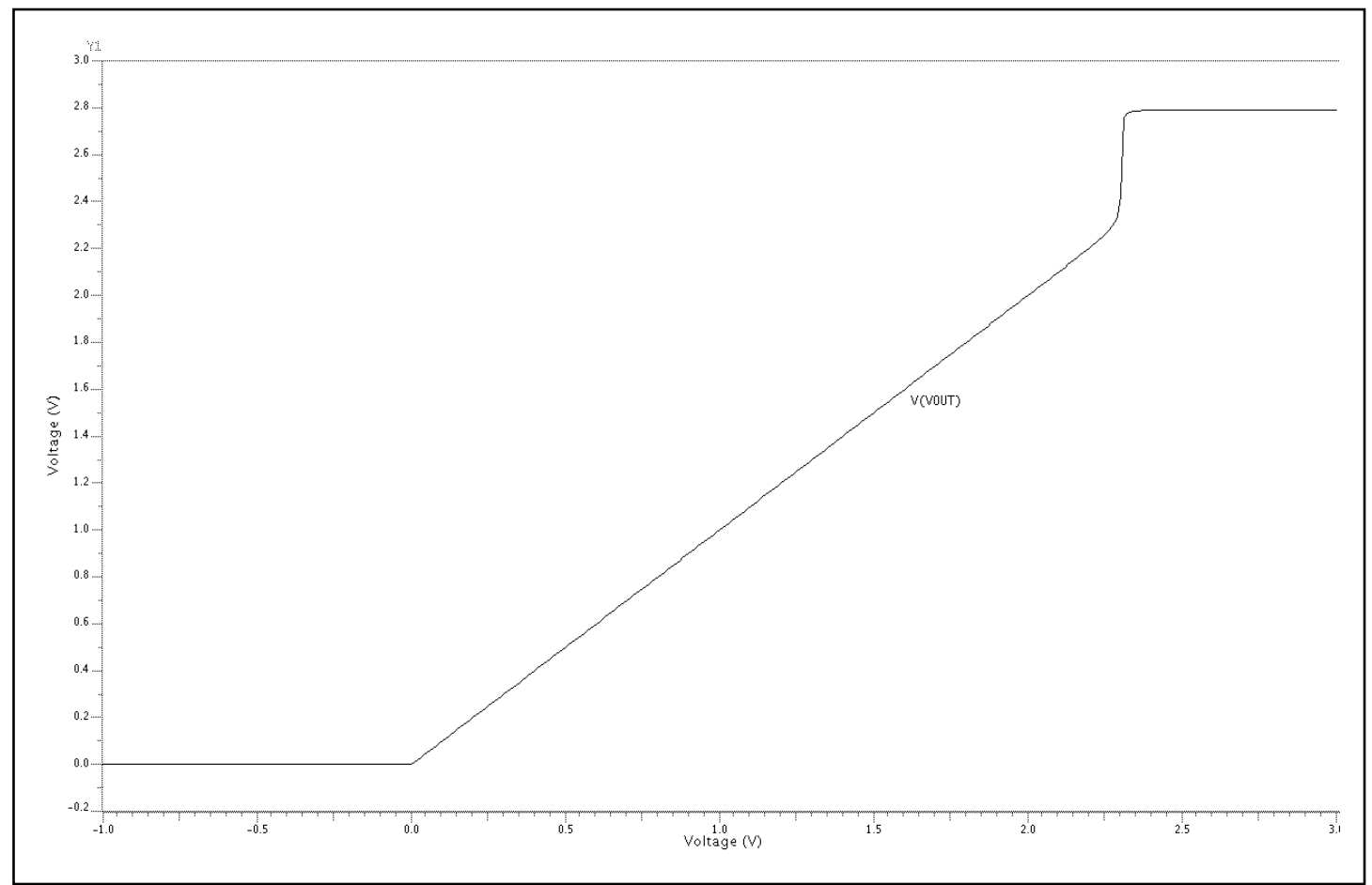

Figura 5.2. Simulação de ICMR do amplificador folded cascode para o caso típico, $40^{\circ} \mathrm{C}, I_{B I A S}=$ $1,5 \mu A$.

Podemos verificar que a região linear de operação se encontra entre os limites $[0 ; 2,3] \mathrm{V}$.

\subsection{Excursão do Sinal de Saída}

A excursão do sinal de saída se mostra bastante importante no que diz respeito à linearidade do sinal fornecido pelo amplificador. Quanto mais próximo das tensões de alimentação, VDD e VSS, estiver o sinal de saída, mais os transistores deixarão a região de saturação, acarretando em distorção harmônica. A figura 5.3 apresenta o testbench utilizado para as medidas de excursão do sinal de saída.

Os resistores $R_{1}$ e $R_{2}$ foram escolhidos de igual valor de forma a fornecer um ganho de malha fechada igual à 1 . Vale ressaltar que nesta simulação a tensão da entrada positiva do ampOp está fixada e é igual à tensão de referência $V_{R E F}$. Dessa forma, apenas a tensão na entrada negativa do ampOp irá variar. Um outro ponto a destacar é que a excursão do sinal de saída varia de forma linear praticamente até atingir os rails, o que caracteriza o estágio de saída do ampOp como rail-to-rail.

A simulação foi realizada através de uma varredura $D C$ da fonte de entrada $V_{\text {in }}$ entre $[-0,3 ; 3] \mathrm{V}$. 


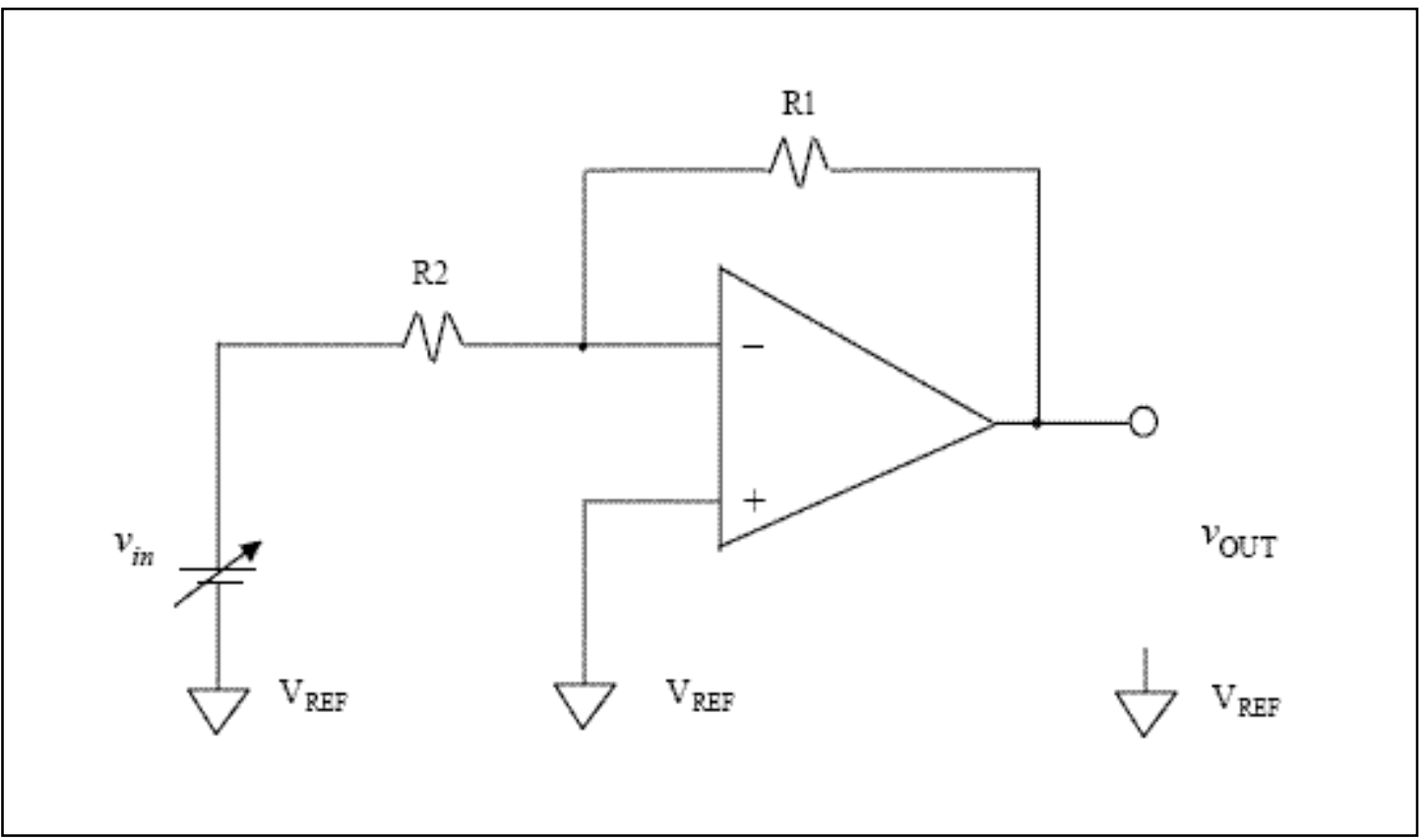

Figura 5.3. Testbench utilizado para simulação e medida de excursão do sinal de saída.

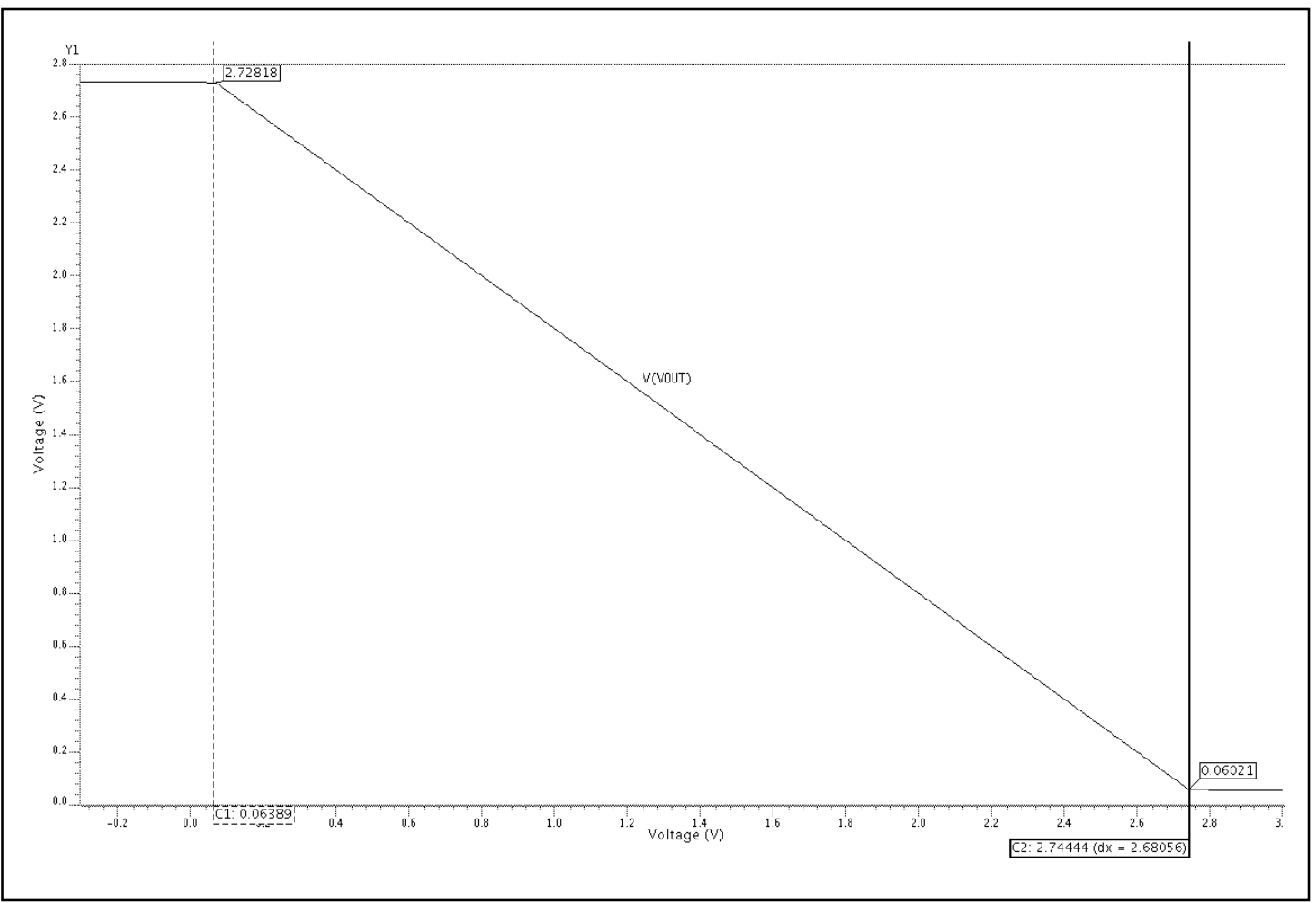

Figura 5.4. Simulação da excursão de sinal de saída para o caso típico, $40{ }^{\circ} \mathrm{C}, I_{B I A S}=1,5 \mu A$.

O gráfico da figura 5.4 identifica os dois extremos que limitam a faixa de operação linear do sinal na saída, sendo estes $[0,06 ; 2,74] \mathrm{V}$, quase atingindo os rails. 


\subsection{Resposta em Freqüência}

A resposta em freqüência do amplificador é, em muitos casos, a mais importante caracterização do circuito, pois fornece o ganho de malha aberta, a banda GBW e a margem de fase responsável pela estabilidade do amplificador.

A figura 5.5 apresenta o testbench utilizado para realização da simulação $A C$ do circuito. Vale a pena destacar que essa topologia é adequada apenas para simulação, sendo necessário aprimorá-la para realizar as medidas experimentais no circuito fabricado, o que será apresentado no próximo capítulo.

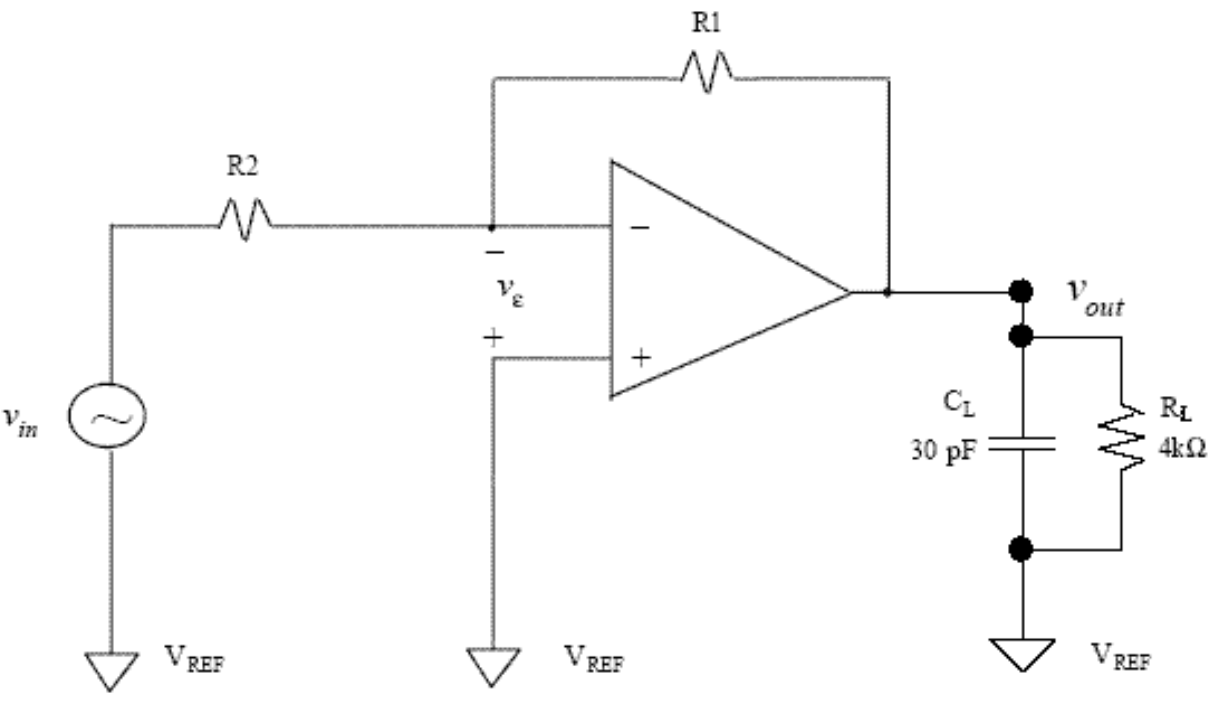

Figura 5.5. Testbench utilizado para simulação da resposta em freqüência do amplificador.

Foi realizada uma simulação $A C$ do circuito e a resposta em freqüência do amplificador é apresentada na figura 5.6. Os parâmetros mais importantes desse gráfico são apresentados na tabela 5.1 .

Tabela 5.1. Parâmetros retirados do gráfico da simulação AC do amplificador.

\begin{tabular}{|c|c|c|}
\hline Parâmetro & Valor & Unidade \\
\hline $\mathrm{A}_{\mathrm{V}}$ & 81,4 & $\mathrm{~dB}$ \\
\hline $\mathrm{fp}_{1}$ & 310 & $\mathrm{~Hz}$ \\
\hline$G B W$ & 3,3 & $\mathrm{MHz}$ \\
\hline $\mathrm{MF}$ & 61,5 & $\circ$ \\
\hline
\end{tabular}




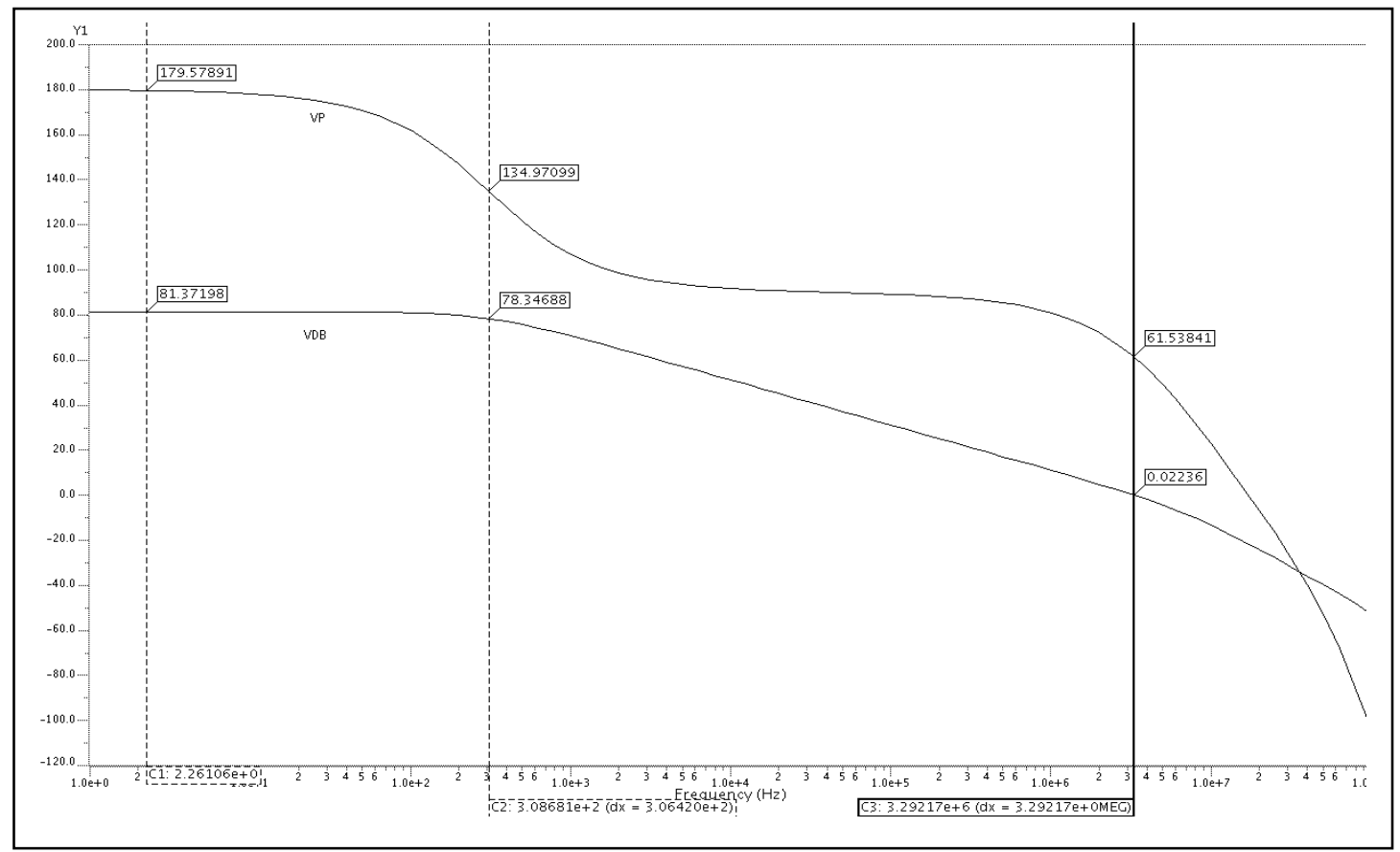

Figura 5.6. Resultado da simulação AC do circuito da figura 5.5, para o caso típico, $40{ }^{\circ} \mathrm{C}, I_{B I A S}=$ $1,5 \mu \mathrm{A}$.

\subsection{Common Mode Rejection Ratio - CMRR}

A Rejeição de Modo Comum (Common Mode Rejection Ratio - CMRR) representa o quanto o circuito projetado amplifica a entrada diferencial e não a de modo comum. Assim ruídos que ocorram na tensão de modo comum devem ser rejeitados. A figura 5.7 apresenta o testbench utilizado para simulação dessa característica [8].

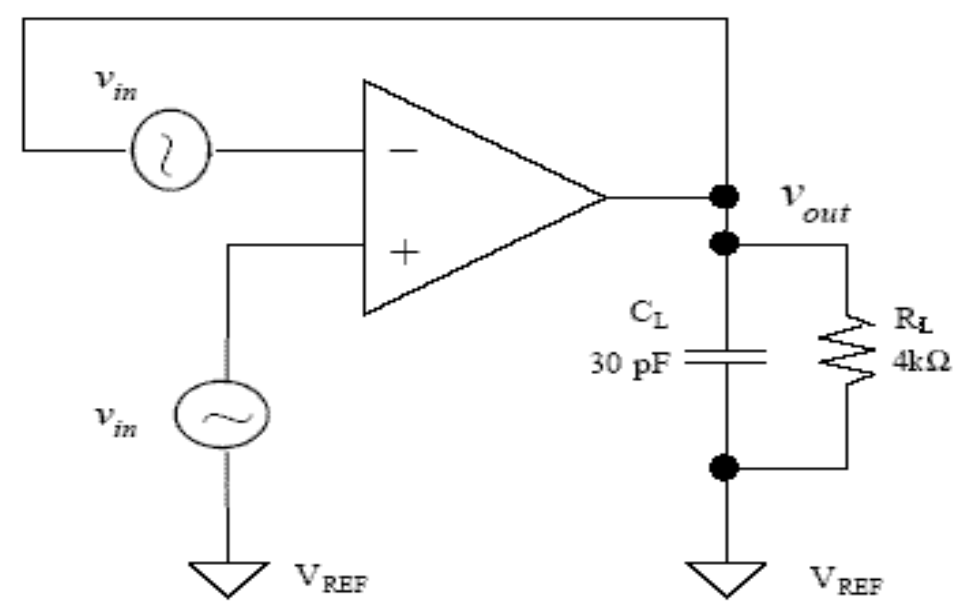

Figura 5.7. Testbench utilizado para simulação de $C M R R$. 
Colocando uma fonte de sinal nas duas entradas do amplificador, pode-se mostrar que [8],

$$
\frac{v_{\text {out }}}{v_{\text {in }}} \cong \frac{\left|A_{C M}\right|}{A_{V}}=\frac{1}{C M R R}
$$

A figura 5.8 apresenta o resultado da simulação acima.

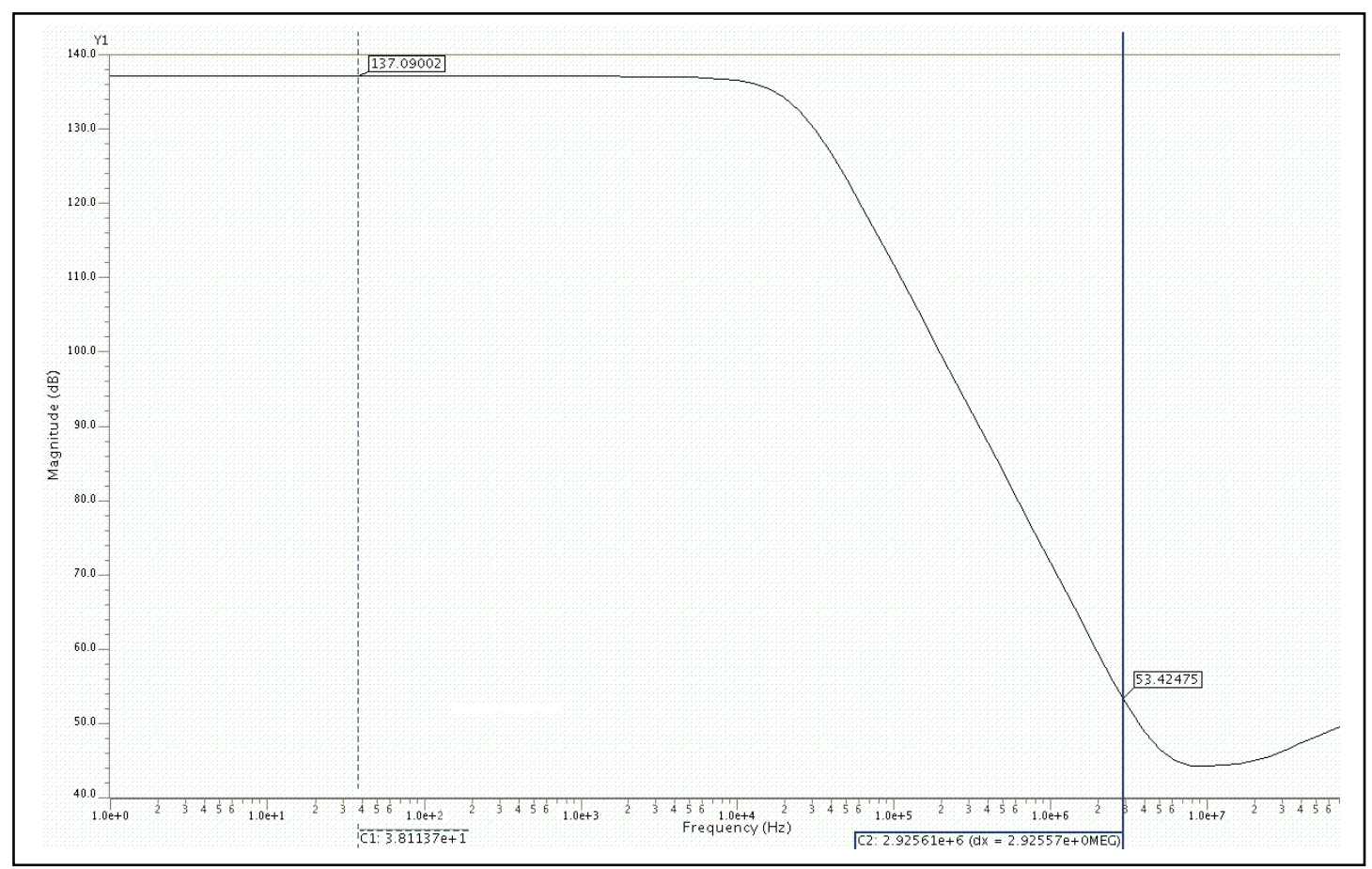

Figura 5.8. Resultado da simulação para verificação de $C M R R$, para o caso típico, $40{ }^{\circ} \mathrm{C}, I_{B I A S}=$ $1,5 \mu A$.

Os parâmetros mais importantes desse gráfico são apresentados na tabela 5.2.

Tabela 5.2. Valores de CMRR extraídos do gráfico da figura 5.8.

\begin{tabular}{|c|c|c|}
\hline Parâmetro & Valor & Unidade \\
\hline CMRR (baixas freq.) & 137 & $\mathrm{~dB}$ \\
\hline CMRR (GBW) & 53 & $\mathrm{~dB}$ \\
\hline
\end{tabular}

\subsection{Power Supply Rejection Ratio - PSRR}

Nesta seção será verificado qual o valor para a Rejeição de Fonte de Alimentação (Power Supply Rejection Ratio - PSRR) do amplificador folded cascode. Da literatura [6] [8] sabemos que os transistores de saída, através das capacitâncias parasitas $C_{g s}$, são os maiores responsáveis pela transmissão de ruídos, presentes nas fontes de alimentação, para a saída, principalmente em altas freqüências. Dessa forma, pensando em maximizar o PSRR, deve-se realizar o layout dos transistores 
de saída de modo a minimizar essas capacitâncias, utlizando-se para isso a menor área $W \cdot L$ possível e layout tipo finger [12].

A figura 5.9 apresenta o testbench utilizado para simulação do PSRR. Foi introduzido um sinal na alimentação do circuito, $V D D$, e realizada uma simulação $A C$.

O resultado dessa simulação é apresentado na figura 5.10.

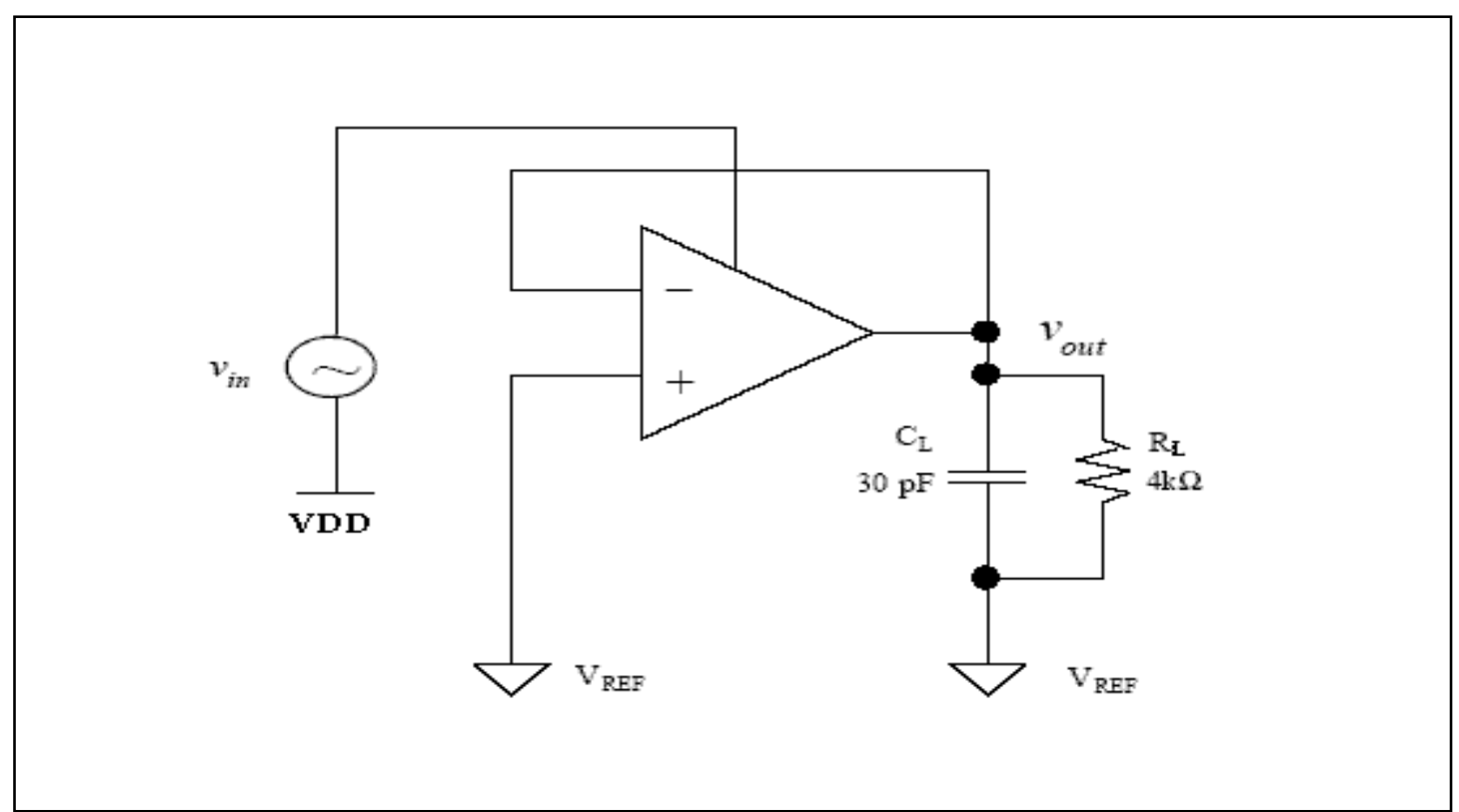

Figura 5.9. Testbench utilizado para simulação de $P S R R$.

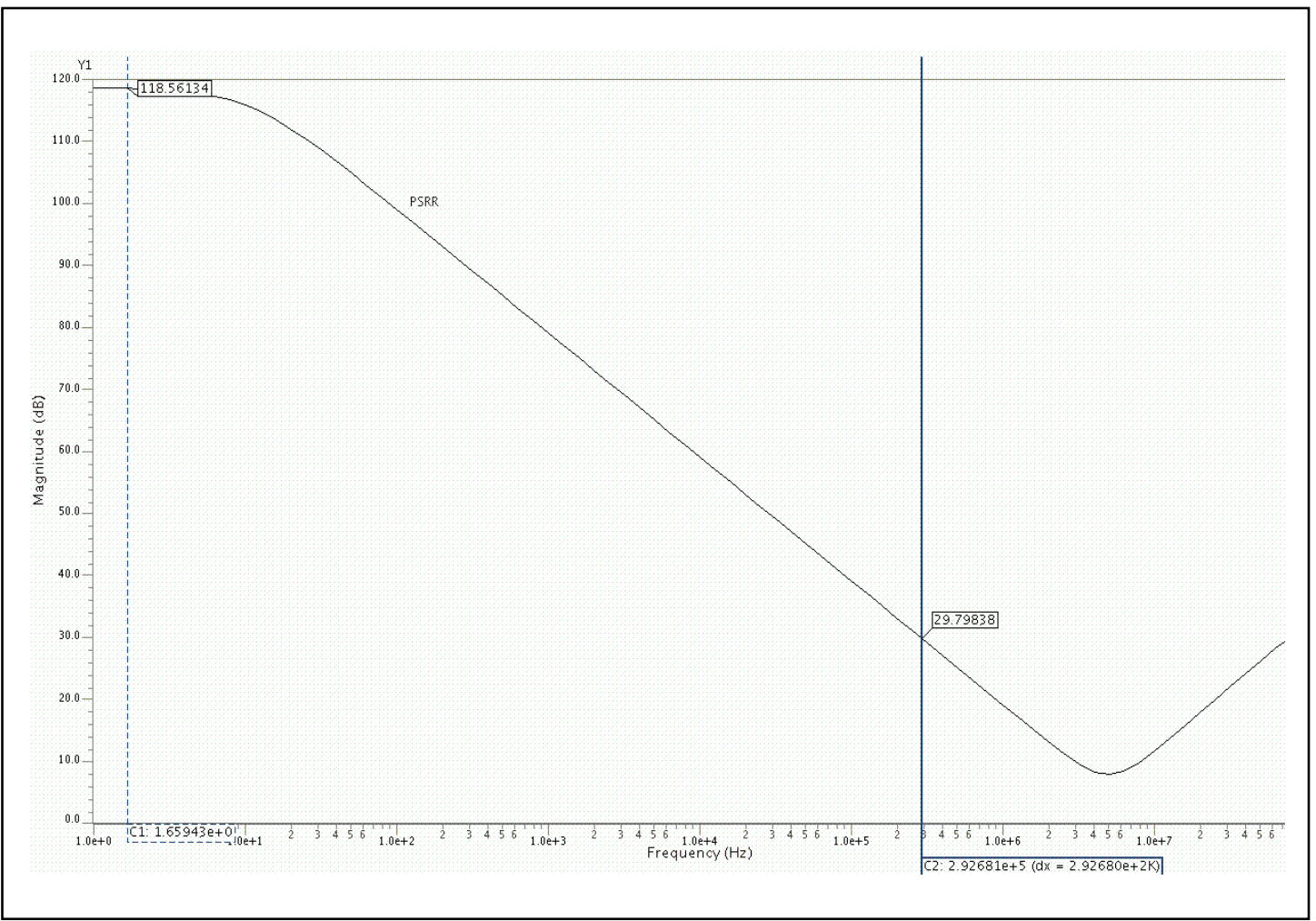

Figura 5.10. Resultado da simulação de $P S R R$, para o caso típico, $40^{\circ} \mathrm{C}, I_{B I A S}=1,5 \mu A$. 
Os parâmetros mais importantes desse gráfico são apresentados na tabela 5.3.

Tabela 5.3. Valores de PSRR extraídos do gráfico da figura 5.10.

\begin{tabular}{|c|c|c|}
\hline Parâmetro & Valor & Unidade \\
\hline PSRR (B.F.) & 118,6 & $\mathrm{~dB}$ \\
\hline PSRR (GBW) & 29,8 & $\mathrm{~dB}$ \\
\hline
\end{tabular}

\subsection{Slew Rate}

A medida do slew rate, $S R$, determina qual a maior taxa de variação a tensão de saída pode ter. Normalmente é dado em $V / \mu$ s. A resposta do circuito será distorcida quando for exigido que sua variação seja mairor do que o $S R$, pois não é possível carregar e descarregar as capacitâncias associadas aos nós tão rapidamente quanto o necessário.

A figura 5.11 apresenta o testbench utilizado para simulação do slew rate do circuito.

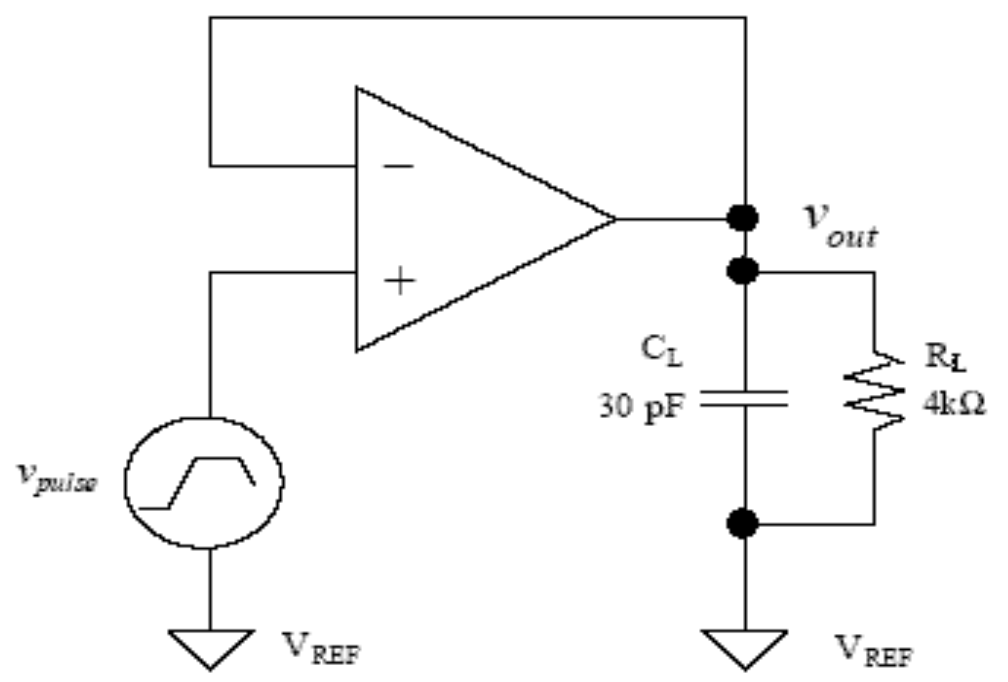

Figura 5.11. Testbench utilizado para simulação e medida de slew rate.

O sinal introduzido pela fonte $v_{\text {pulse }}$ deve ser suficientemente grande para que o amplificador não consiga acompanhar essa variação e possa ser verificada a 
distorção causada no sinal de saída. A figura 5.12 apresenta o resultado da simulação em conjunto com o pulso de entrada, que variou de $1,4 \mathrm{~V}$ à 1,8 $\mathrm{V}$. Podese notar claramente a distorção do sinal de saída. O valor do $S R$ está apresentado na tabela 5.4 .

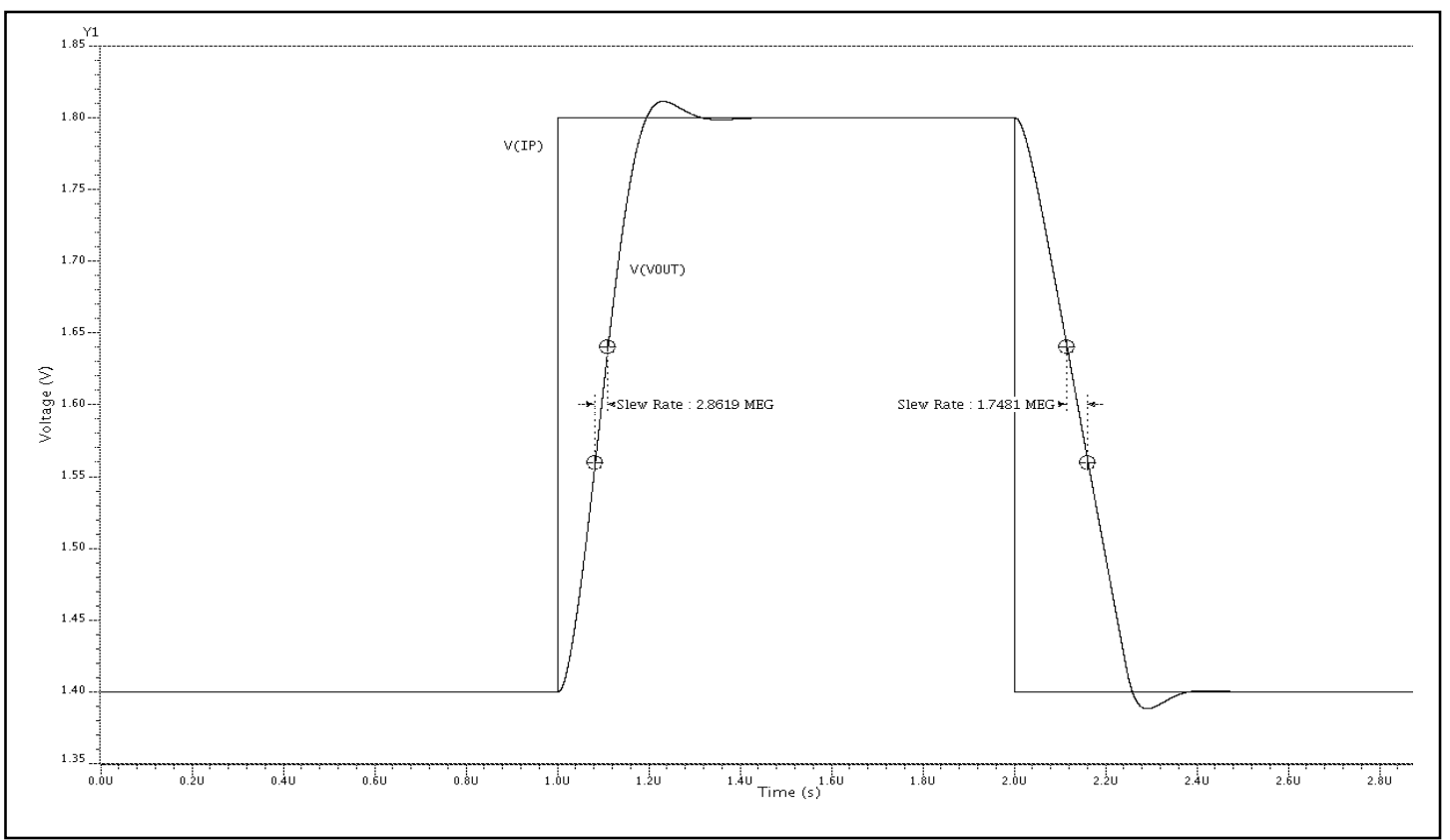

Figura 5.12. Pulso de entrada e resposta do circuito, para verificação do $S R$, para o caso típico, $40{ }^{\circ} \mathrm{C}, I_{B I A S}=1,5 \mu A$.

Tabela 5.4. Valores de $S R$ extraídos do gráfico da figura 5.12.

\begin{tabular}{|c|c|c|}
\hline Parâmetro & Valor & Unidade \\
\hline$S R$ (positivo) & 2,86 & $V / \mu s$ \\
\hline$S R$ (negativo) & 1,75 & $V / \mu s$ \\
\hline
\end{tabular}




\section{Capítulo 6}

\section{Medidas e Resultados Experimentais}

Uma vez finalizado o projeto do amplificador operacional folded cascode, deu-se início ao seu layout. Alguns cuidados devem ser tomados durante a realização do layout, que são [8] [12]:

- Minimizar os erros randômicos, inerentes ao processo de fabricação, através do correto posicionamento dos transistores;

- Melhorar o casamento de transistores importantes, como o par diferencial de entrada, utilizando a técnica common-centroid, o que também visa minimizar eventuais gradientes que possam existir (como por exemplo, de temperatura);

- Utilizar transistores unitários para melhorar casamento de espelhos de corrente;

- Manter o projeto e o desenho o mais simétrica possível, minimizando o offset sistemático;

- Atentar para os componentes que compõe as redondezas dos transistores, utilizando transistores dummy para melhorar o casamento.

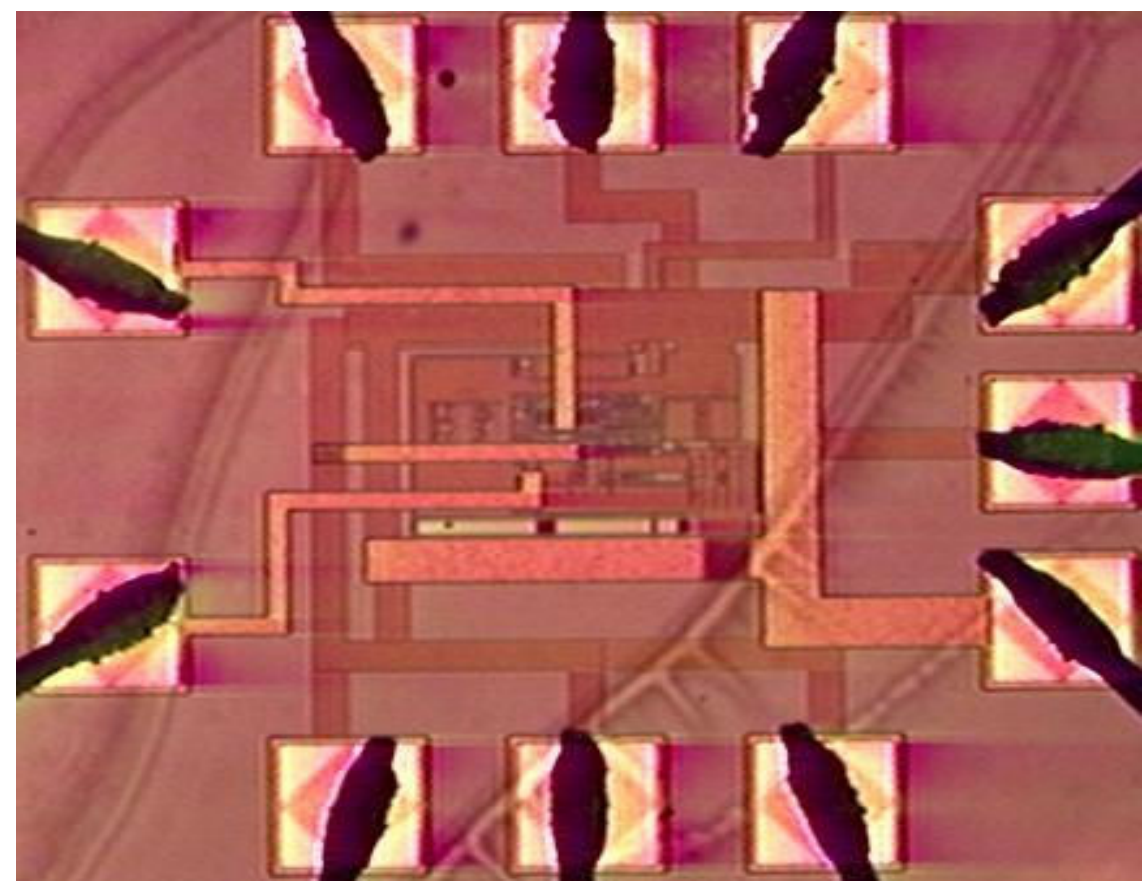

Figura 6.1. Fotomicrografia do ampOp folded cascade fabricado. 


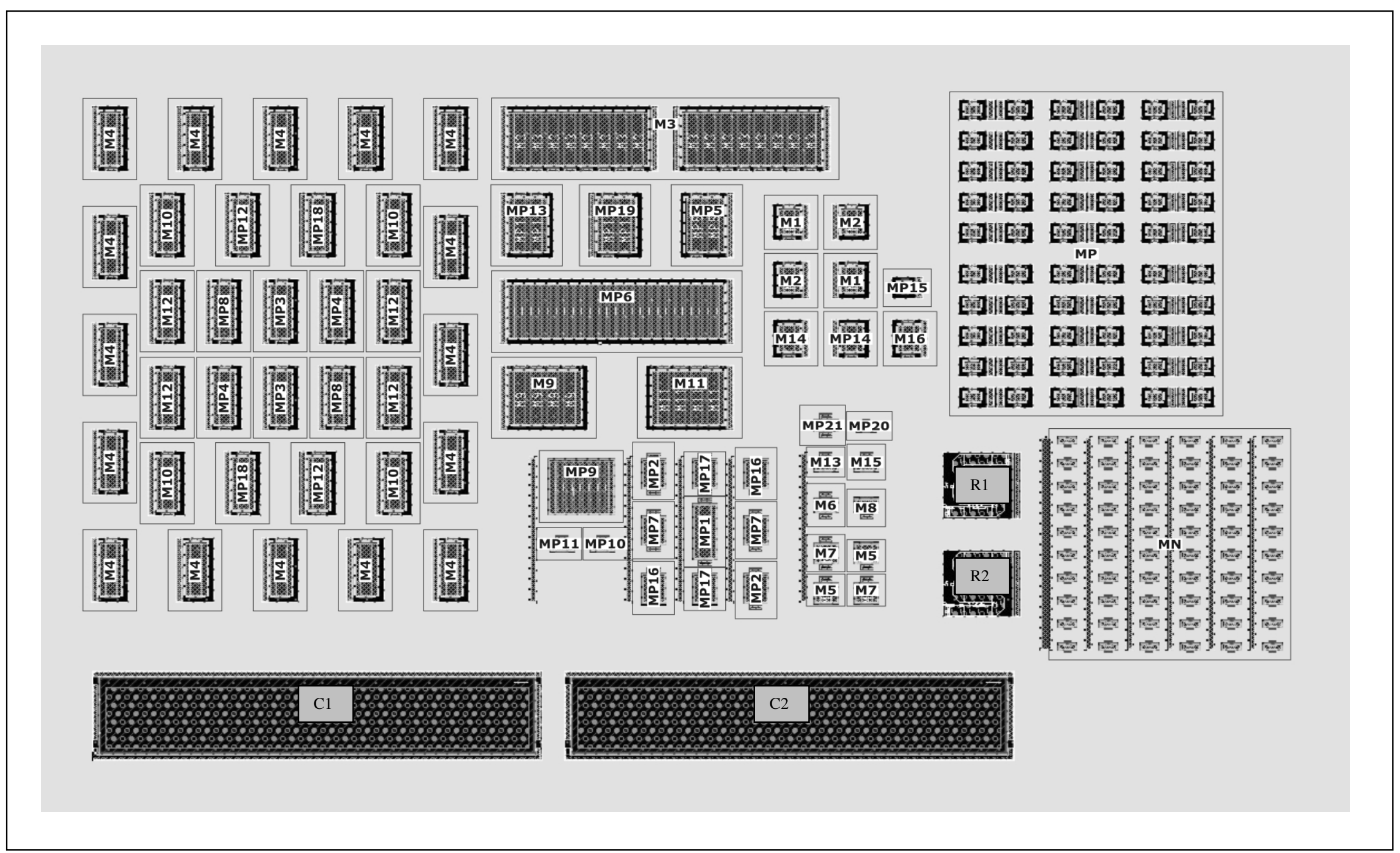

Figura 6.2. Layout do ampOp folded cascode. Os transistores estão identificados. 
A figura 6.1 apresenta a fotomicrografia do circuito projetado e a posição dos pads. A área total do circuito, incluindo os pads, é de $675 \mu m \times 590 \mu m$. Sem considerar os pads a área é de $212 \mu \mathrm{m} \times 127 \mu \mathrm{m}$. Já a figura 6.2 apresenta o layout do ampOp, onde é possível verificar a posição dos transistores e a técnica commoncentroid utilizada para melhorar o casamento entre, por exemplo, os transistores de entrada $M 1$ e M2. Não estão representadas as trilhas de metais utilizadas para o roteamento do circuito, de forma a permitir uma melhor visualização dos transistores.

A caracterização completa do amplificador envolve medidas $D C, A C \mathrm{e}$ transiente. A seguir os resultados dessas medidas serão apresentados.

\subsection{Caracterização DC}

\subsubsection{Medida da tensão de Offset}

A tensão de offset é proveniente de assimetrias no circuito projetado (offset sistemático) e proveniente dos erros estatísticos referentes ao processo de fabricação (offset randômico). O sistemático pode ser bastante minimizado ao se fazer um projeto simétrico do circuito. Já o randômico sempre existirá e pode ser, na melhor das hipóteses, reduzido, mas nunca eliminado. Gradientes de temperatura, de forças de stress no material, variações no crescimento do óxido dos terminais de porta, entre outros, sempre existirão e causarão tais erros.

A figura 6.3 mostra o testbench utilizado para medida do offset do ampOp fabricado.

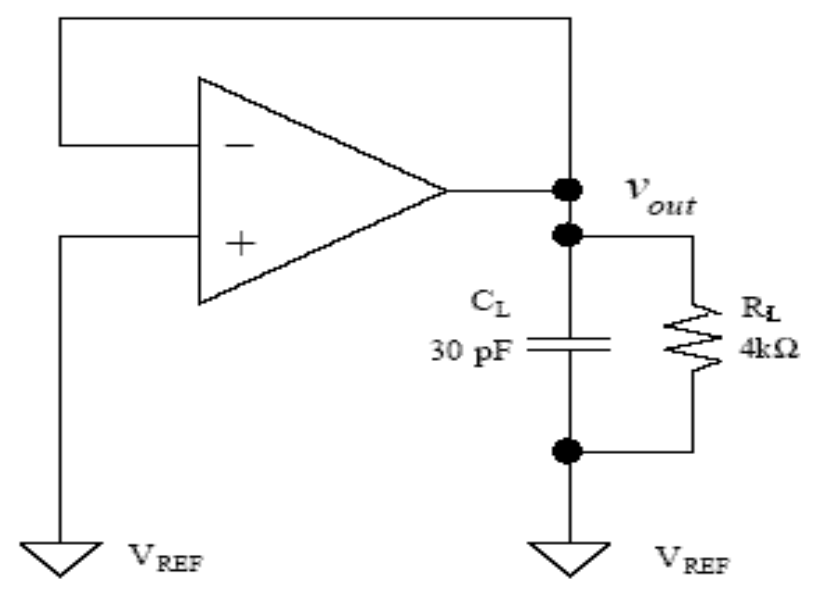

Figura 6.3. Testbench utilizado para medida da tensão de offset de entrada do ampOp. 
Como o offset esperado é bastante pequeno, foi necessário utilizar fontes de referência bastante precisas e estáveis, com precisão de miliVolt. Além disso, foram encapsulados cinco chips, e foram medidos o offset dos cinco. A tabela 6.1 apresenta os resultados das medidas realizadas para o chip $A$, utilizando-se um multímetro, em temperatura ambiente.

Tabela 6.1. Valores medidos das tensões do circuito para cálculo da tensão de offset do chip A.

\begin{tabular}{|c|c|c|}
\hline Parâmetro & Medida & Unidade \\
\hline $\mathrm{V}_{\text {REF }}$ & 1,39973 & $\mathrm{~V}$ \\
\hline $\mathrm{V}_{\text {OUT }}$ & 1,40140 & $\mathrm{~V}$ \\
\hline
\end{tabular}

O valor da tensão de offset pode ser calculada como a diferença entre os valores medidos,

$$
V_{O S}=V_{\text {OUT }}-V_{\text {REF }}=-1,7 m V
$$

A exemplo do cálculo efetuado acima para o chip $A$, o mesmo foi realizado para os demais chips, e os resultados estão consolidados na tabela 6.2.

Tabela 6.2. Tensão de offset dos chips $A, B, C, D$ e $E$.

\begin{tabular}{|c|c|c|}
\hline Chip & Offset & Unidade \\
\hline$A$ & $-1,7$ & \multirow{2}{*}{$\mathrm{V}$} \\
\hline$B$ & 8 & \\
\hline$D$ & -22 & \\
\hline$E$ & 40 & \\
\hline
\end{tabular}

Percebe-se uma variação significativa entre os valores medidos. Não foi encontrada nenhuma razão para valores tão altos de offset, sendo que o cálculo das variações randômicas do processo fornece um resultado para o desvio padrão de $\sigma \approx 5 m V$ [17], [21]. Considerando que 99\% das amostras se encontram, estatisticamente, dentro de um intervalo de $3 \cdot \sigma \approx 15 \mathrm{mV}$ em torno da média, essa variação randômica ainda não justifica tal problema.

As demais medidas foram realizadas apenas para o chip $A$.

\subsubsection{Medida de Input Common Mode Range - ICMR}

O testbench da figura 6.4 foi utilizado para medição do ICMR do amplificador. Nessa montagem foi realizada uma varredura $D C$ da fonte de entrada $V_{\text {in }}$ entre [-0,5 ;2,8] $V$, verificando-se o valor de tensão na saída do circuito. A faixa de operação de interesse corresponde à parte linear do gráfico da figura 6.5 . 
Capítulo 6-Medidas e Resultados Experimentais

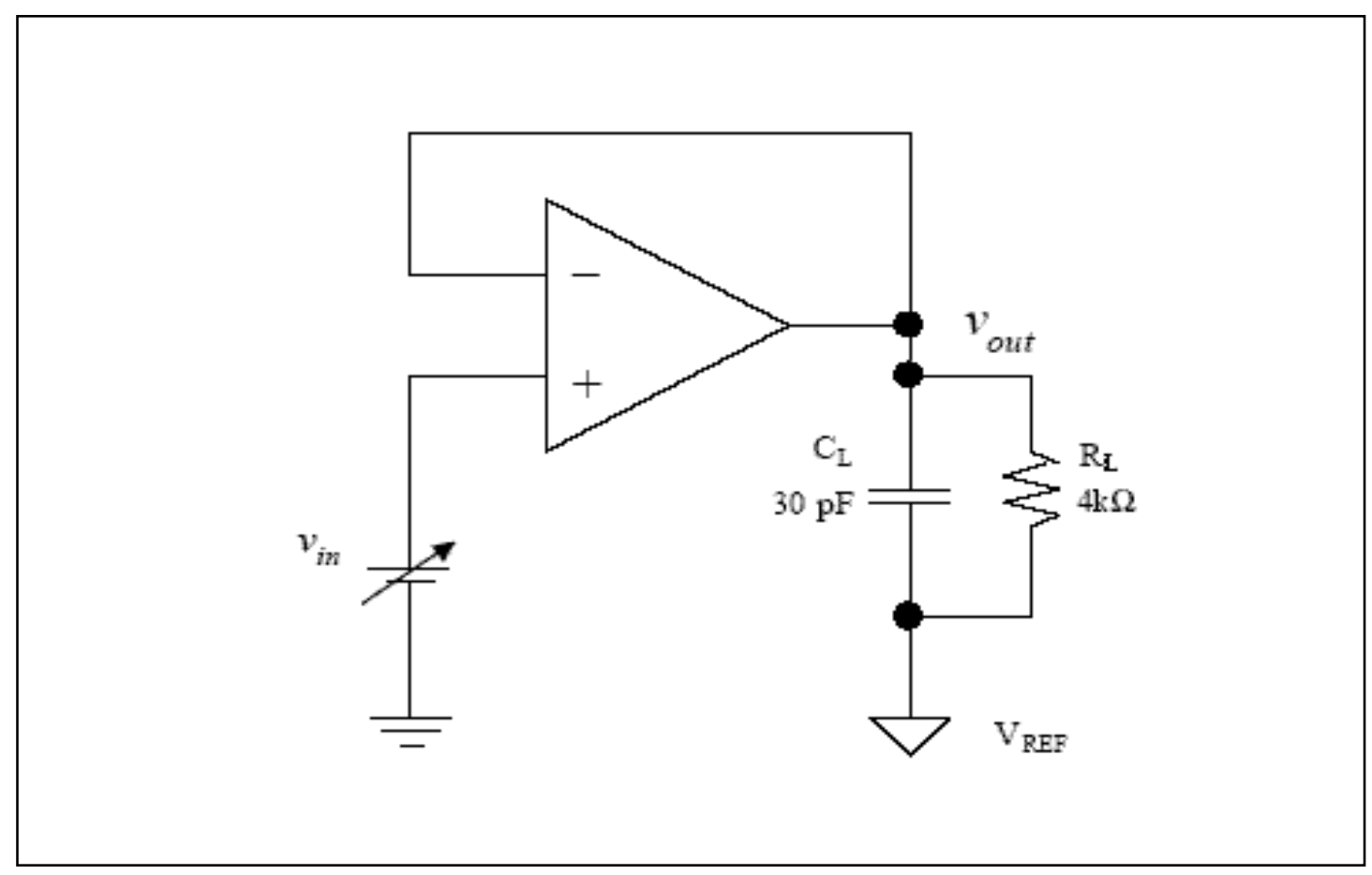

Figura 6.4. Testbench utilizado para medida de ICMR.

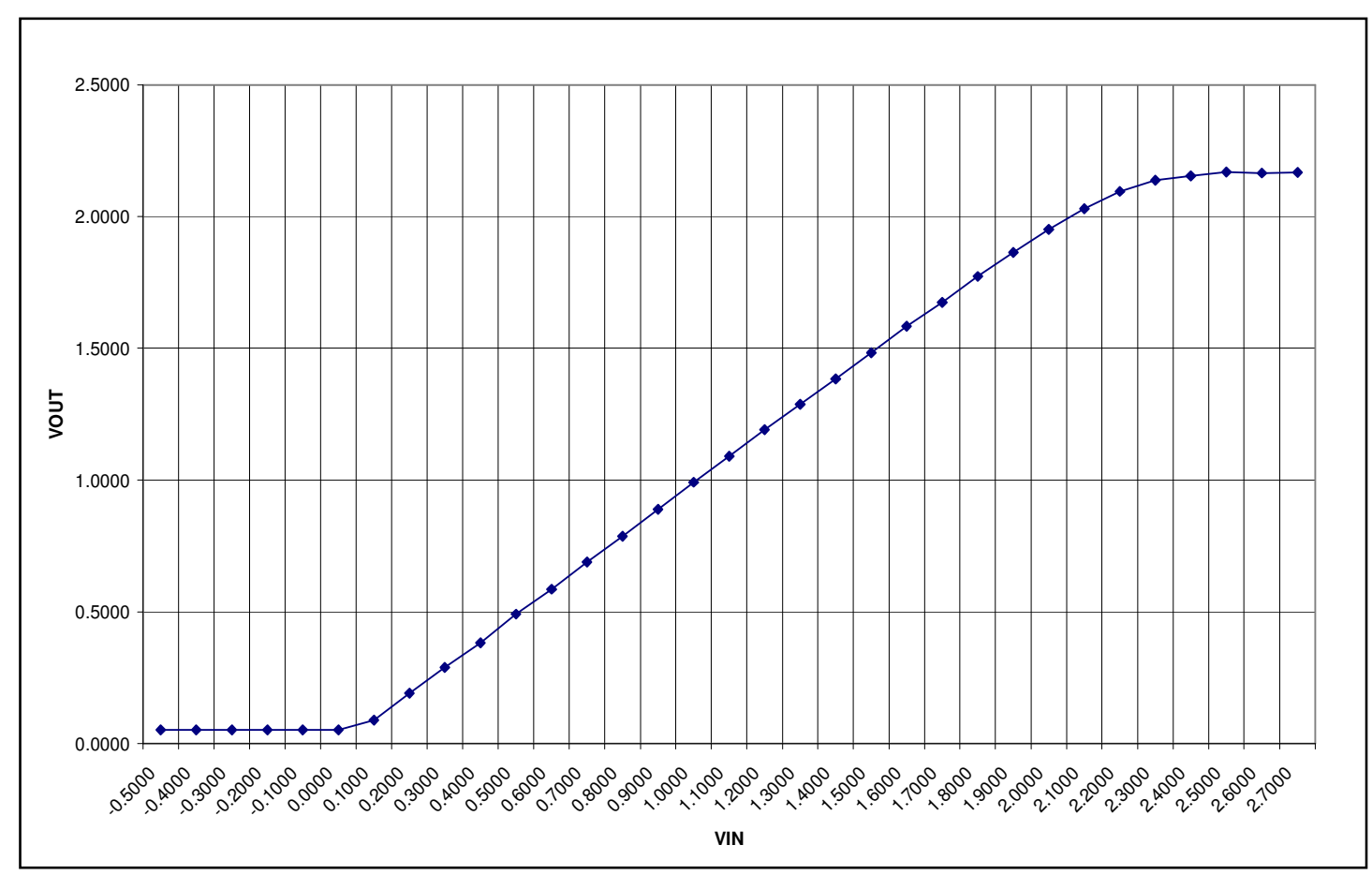

Figura 6.5. ICMR medido do amplificador folded cascode.

65 


\subsubsection{Medida de Excursão do Sinal de Saída}

A figura 6.6 apresenta o testbench utilizado para as medidas de excursão do sinal de saída. Os resistores $R_{1}$ e $R_{2}$ foram escolhidos de mesma magnitude de forma a fornecer um ganho de malha fechada igual à 1. A tabela 6.3 apresenta os valores dos resistores utilizados.

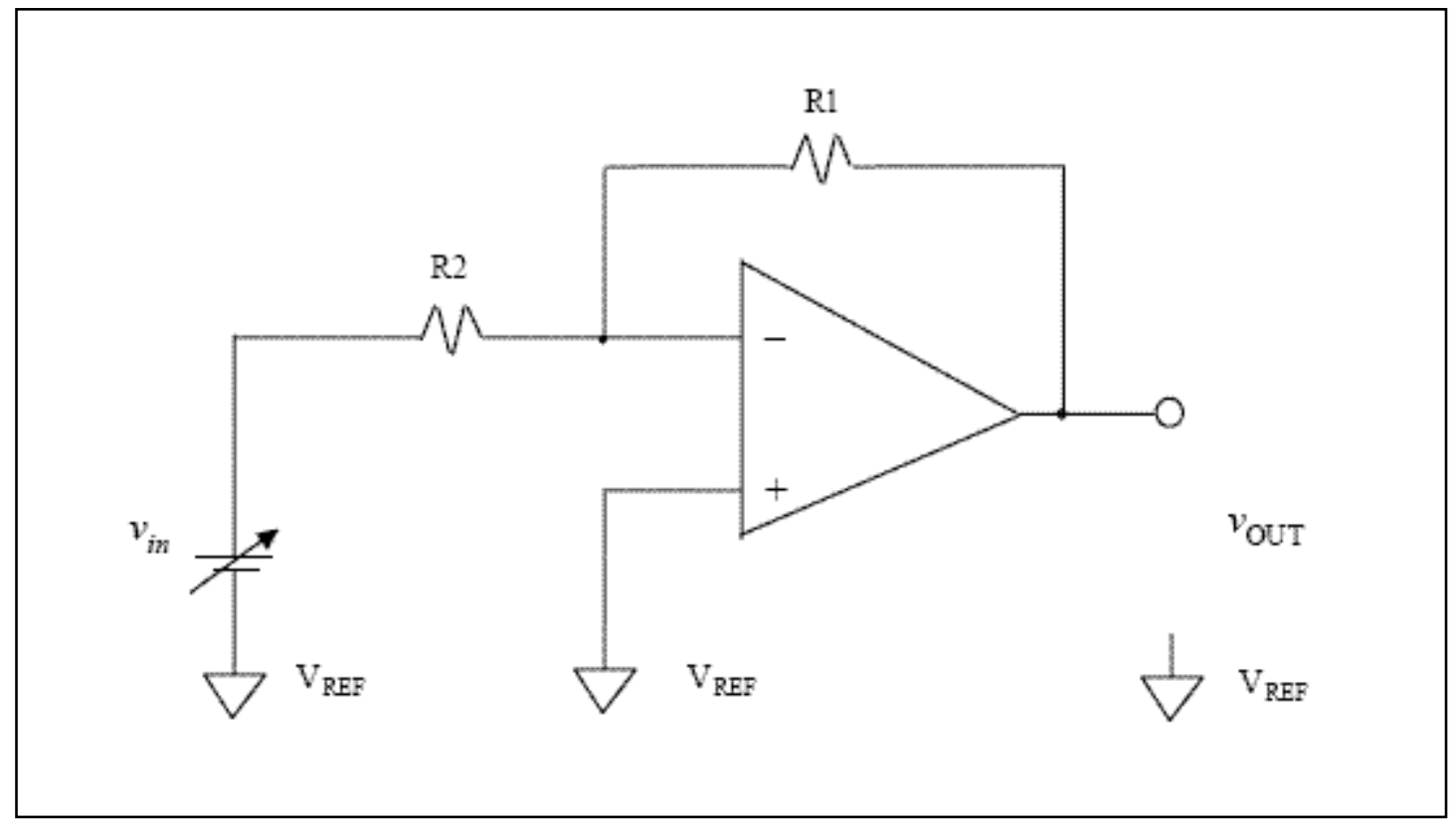

Figura 6.6. Testbench utilizado para medida de excursão do sinal de saída.

Tabela 6.3. Resistores utilizados na montagem do testbench da figura 6.6.

\begin{tabular}{|c|c|c|}
\hline Grandeza & Valor & Unidade \\
\hline$R_{1}$ & 100 & $\mathrm{k} \Omega$ \\
\hline $\mathrm{R}_{2}$ & 100 & $\mathrm{k} \Omega$ \\
\hline
\end{tabular}

Foi realizada uma varredura $D C$ da fonte de entrada $V_{\text {in }}$ entre $[-0,8 ; 3] V$, e os resultados das medidas são apresentados no gráfico da figura 6.7. 


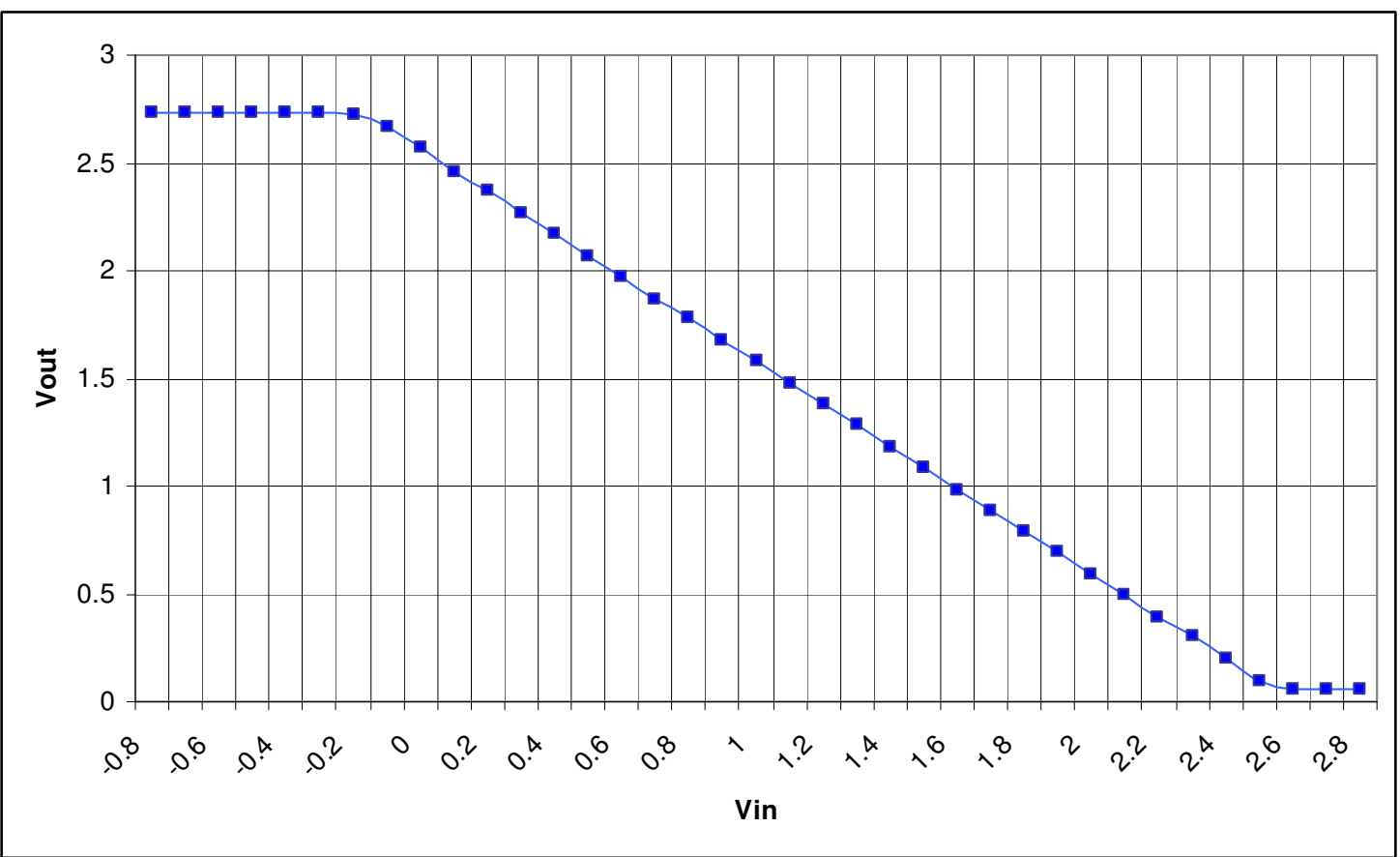

Figura 6.7. Medida da excursão de sinal de saída do amplificador.

\subsection{Caracterização AC}

\subsubsection{Medida do Ganho de tensão de malha aberta - $A_{V}$}

Dentre todas as medidas necessárias para a caracterização do amplificador operacional, a medida do ganho de malha aberta talvez a mais complicada de realizar, especialmente se o ganho do amplificador for alto como em nosso caso. Para se obter o ganho do circuito é necessários medir os valores de (figura 6.8):

- $\mathrm{v}_{\mathrm{o}}$ : tensão de saída, geralmente da ordem de Volt, sendo facilmente medida;

- $v_{\mathrm{e}}$ : tensão diferencial de entrada, da ordem de $\mu$ Volt , que apresenta grande dificuldade para ser obtida.

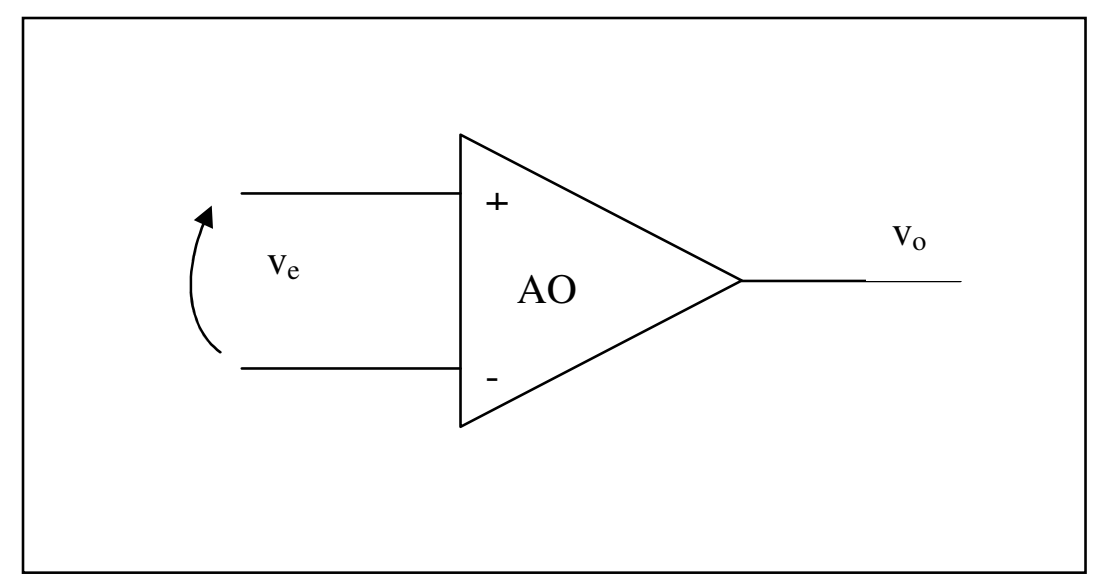

Figura 6.8. Tensões necessárias para o cálculo do ganho de malha aberta do amplificador operacional. 
Pelo fato da tensão diferencial de entrada, $v_{e}$, ser muito pequena, torna-se inviável medí-la diretamente, sendo, portanto, necessário amplificá-la. As figuras 6.9 e 6.10 apresentam a topologia de medida utilizada para a caracterização do ganho de malha aberta do operacional.

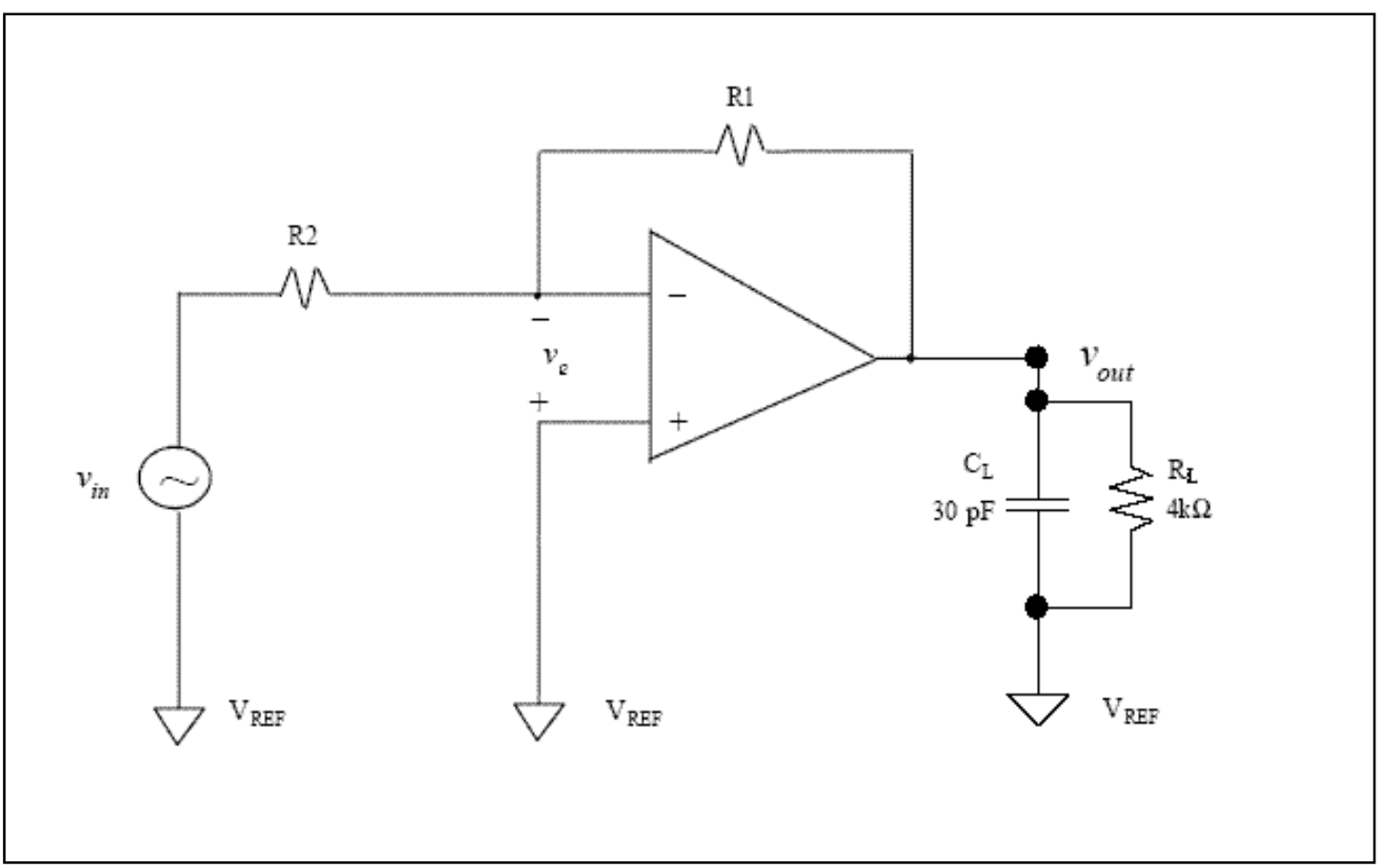

Figura 6.9. Testbench utilizado para medida do ganho de malha aberta.

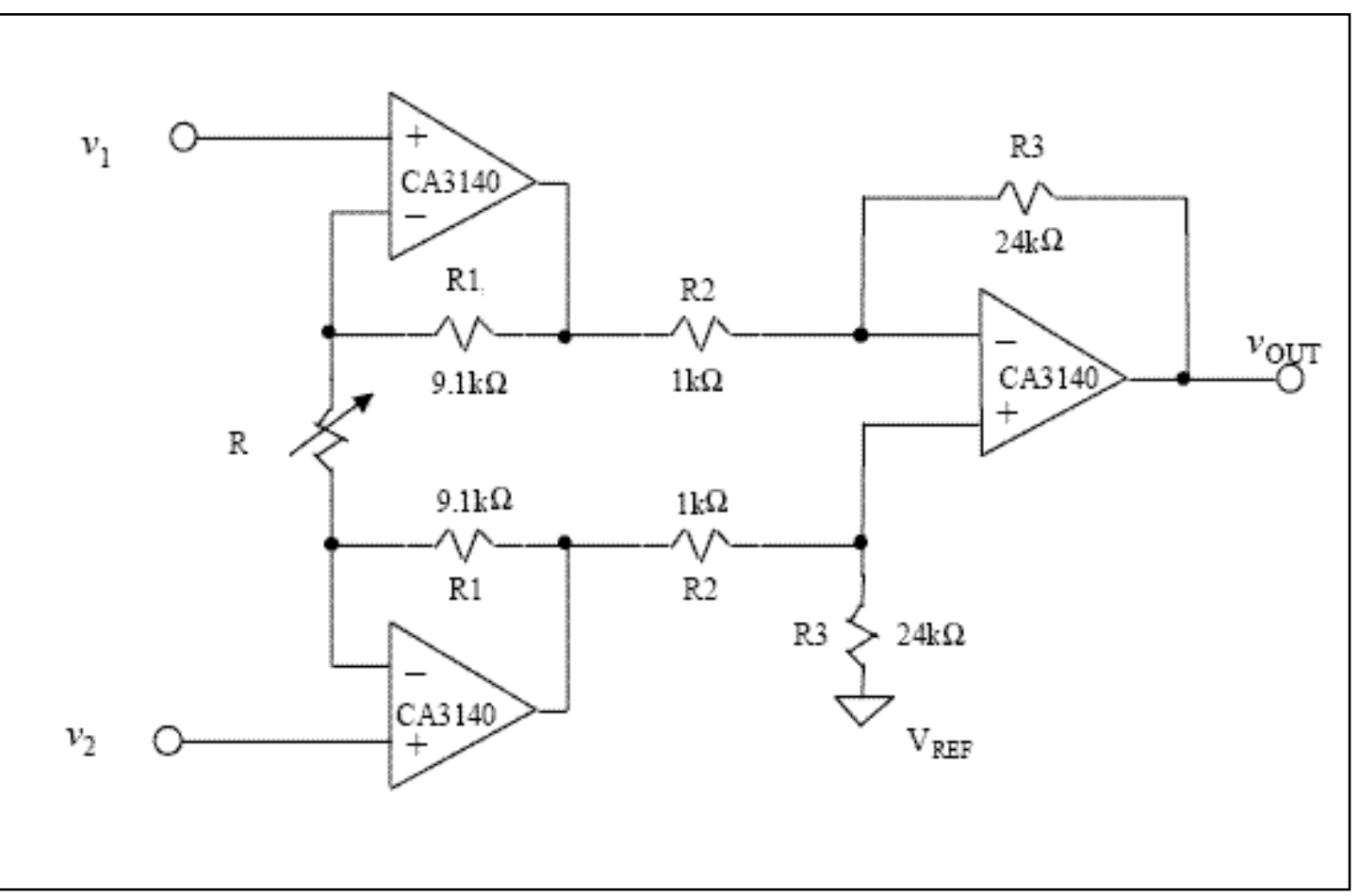

Figura 6.10. Amplificador de Instrumentação (in-amp) utilizado para amplificação do sinal diferencial $v_{e}$. 
A topologia da figura 6.9 apresenta o ampOp realimentado, com ganho de malha fechada dado pela relação entre os resistores $R_{1}$ e $R_{2}$, com inversão de fase. Para que a malha de realimentação não carregue a saída do amplificador, foi utilizado um operacional adicional, o CA3140A, na configuração seguidor de tensão.

A figura 6.10 apresenta o amplificador de instrumentação, in-amp, que será utilizado para amplificar o sinal diferencial $v_{e}$ do circuito da figura 6.9. Foi escolhido este tipo de amplificador, pois sua impedância de entrada é muito grande (entrada MOS), o que contribui para isolar o circuito medido do circuito medidor. Outra característica importante é que ele amplifica tensões diferenciais, o que é necessário para o nosso caso [14] [16]. Vale a pena ressaltar que todos os operacionais utilizados CA3140A tiveram suas tensões de offset canceladas através de potenciômetros.

Vamos agora analisar e projetar o in-amp. Supondo um ganho para o ampOp fabricado de $80 \mathrm{dBs}$ (resultado proveniente da simulação), e uma excursão de $2 V_{p p}$ na saída, o sinal diferencial na entrada do operacional seria de 0,2 $m V_{p p}$, que se encontra abaixo da resolução de grande parte dos equipamentos de medidas. Logo, admitindo um ganho de $500 \mathrm{~V} / \mathrm{V}$ para o in-amp, teríamos na sua saída o sinal $v_{e}$ amplificado para 0,1 $V_{p p}$, que pode ser facilmente medido. $O$ ganho do amplificador de instrumentação é dado por [14] [16],

$$
\frac{v_{\text {out }}}{\left(v_{2}-v_{1}\right)}=2 \cdot \frac{R_{1} \cdot R_{3}}{R \cdot R_{2}}
$$

No lugar de $R$ é utilizado um potenciômetro para que se possa ajustar o ganho com precisão. Ele é ajustado para que o amplificador forneça o ganho de $500 \mathrm{~V} / \mathrm{V}$. A tabela 6.4 mostra os valores usados.

Tabela 6.4. Valores dos resistores que formam o amplificador de instrumentação.

\begin{tabular}{|c|c|c|}
\hline Resistor & Valor & Unidade \\
\hline R1 & 9,1 & $\mathrm{k} \Omega$ \\
\hline R2 & 1 & $\mathrm{k} \Omega$ \\
\hline R3 & 24 & $\mathrm{k} \Omega$ \\
\hline R & 935 & $\Omega$ \\
\hline
\end{tabular}

Uma vez estabelecido o ganho do in-amp, devemos caracterizá-lo para obter seu ganho em função da freqüência do sinal de entrada. Essa caracterização foi realizada, e o gráfico do ganho do amplificador de instrumentação em função da freqüência de entrada é apresentado na figura 6.11 . 


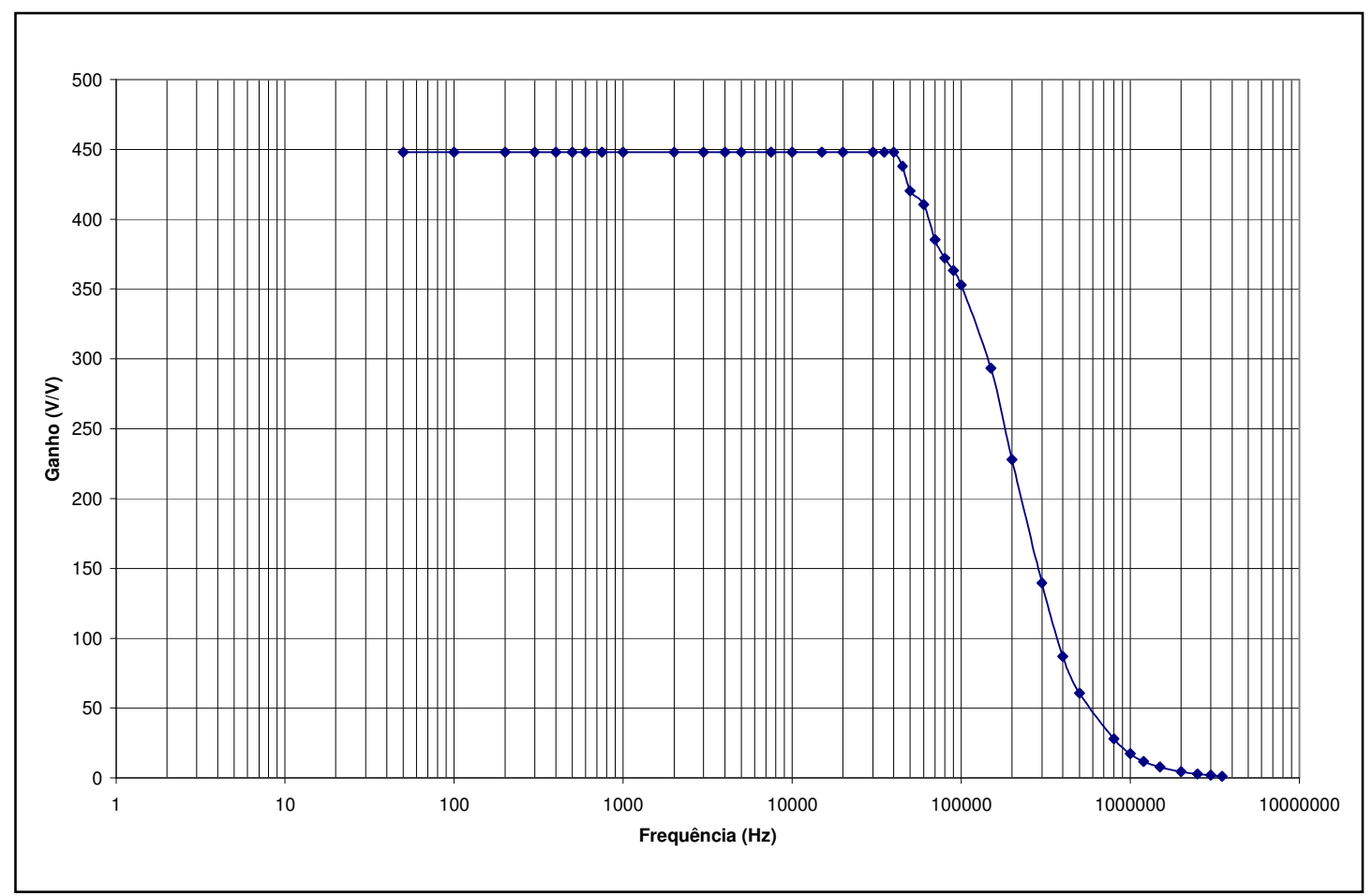

Figura 6.11. Ganho do amplificador de instrumentação em função da freqüência do sinal de entrada.

A tabela 6.5 apresenta os valores medidos do ganho do amplificador de instrumentação. Como o ganho não é constante e varia com a frequência, as medidas amplificadas do sinal $v_{e}$ devem ser corrigidas através dessa curva. Por exemplo, na frequência de $100 \mathrm{kHz}$, se $v_{\text {out }}=50 \mathrm{~m} V_{p p}$ então $v_{e}=\frac{v_{\text {out }}}{\text { ganho(f) }}=\frac{50 \mathrm{~m}}{353}=0,14 \mathrm{mV}$, onde o valor de ganho $(f)=$ ganho $(100 \mathrm{kHz})$ foi retirado da tabela 6.5 .

Tabela 6.5. Valores medidos para o ganho do in-amp em função da freqüência.

\begin{tabular}{|c|c|}
\hline Freqüência (Hz) & Ganho (V/V) \\
\hline $50-40 k$ & 448 (médio) \\
\hline $45 k$ & 438 \\
\hline $50 k$ & 420,2 \\
\hline $60 k$ & 410,5 \\
\hline $70 k$ & 385,3 \\
\hline $80 k$ & 372 \\
\hline $90 k$ & 363,3 \\
\hline $100 k$ & 353 \\
\hline $150 k$ & 293,3 \\
\hline $200 k$ & 228 \\
\hline $300 k$ & 140 \\
\hline $400 k$ & 87 \\
\hline
\end{tabular}




\begin{tabular}{|c|c|}
\hline $500 \mathrm{k}$ & 60,8 \\
\hline $800 \mathrm{k}$ & 28 \\
\hline $1 \mathrm{M}$ & 17,3 \\
\hline $1,2 \mathrm{M}$ & 11,8 \\
\hline $1,5 \mathrm{M}$ & 7,8 \\
\hline $2 \mathrm{M}$ & 4,4 \\
\hline $2,5 \mathrm{M}$ & 2,8 \\
\hline $3 \mathrm{M}$ & 1,9 \\
\hline $3,5 \mathrm{M}$ & 1,3 \\
\hline
\end{tabular}

Um outro problema que surge é o efeito da tensão de offset do ampOp nas medições através do in-amp. Conforme apresentado na seção 6.1.1, a tensão de offset medida se encontra na faixa de $1,7 \mathrm{mV}$. Essa tensão diferencial é amplificada pelo in-amp, com ganho de $448 \mathrm{~V} / \mathrm{V}$, alterando o sinal a ser medido na sua saída.

Vale ainda observar que se essa tensão de offset aumentar, com a temperatura por exemplo, a saída do in-amp poderá inclusive saturar em VDD ou VSS.

Para contornar esse problema, foi introduzido um capacitor de acoplamento no circuito, e a topologia final utilizada para a caracterização do ampOp é apresentada na figura 6.12. A função do capacitor é desacoplar o sinal $D C$ entre a entrada negativa do ampOp e o resistor $R_{2}$. Assim, na saída de $R_{2}$ teremos a tensão $D C$ de $1,4 V$ (referência) somado ao sinal $A C$ presente na entrada negativa do ampOp, eliminando da medida, portanto, o offset. A tensão aplicada ao in-amp para avaliar o ganho será agora $v_{e}$.

É importante ressaltar que com a medida sendo feita a partir de $v_{e}$, não estamos mais apenas com o amplificador, mas sim com o conjunto amplificador e capacitor. O capacitor altera a resposta em freqüência do sistema, pois introduz um novo pólo dado por [14],

$$
f_{p}=\frac{1}{2 \cdot \pi \cdot R_{1} \cdot C}
$$

O circuito passa a apresentar em baixas freqüências a característica de um diferenciador. Logo, para que as medidas do ganho sejam úteis para obtermos o ganho DC do amplificador, devemos escolher a constante $\frac{1}{2 \cdot \pi \cdot R_{1} \cdot C}$ com valor máximo $A_{V}$ vezes menor do que o primeiro pólo do ampOp [8]. Através das simulações, verifica-se que o primeiro pólo do ampOp está em torno de $350 \mathrm{~Hz}$. Com um ganho de $80 \mathrm{dBs}$, temos que, 


$$
\frac{A_{V}}{2 \cdot \pi \cdot R_{1} \cdot C}>f_{P_{1}} \Rightarrow R_{1} \cdot C>4,6 \quad[\Omega \cdot F]
$$

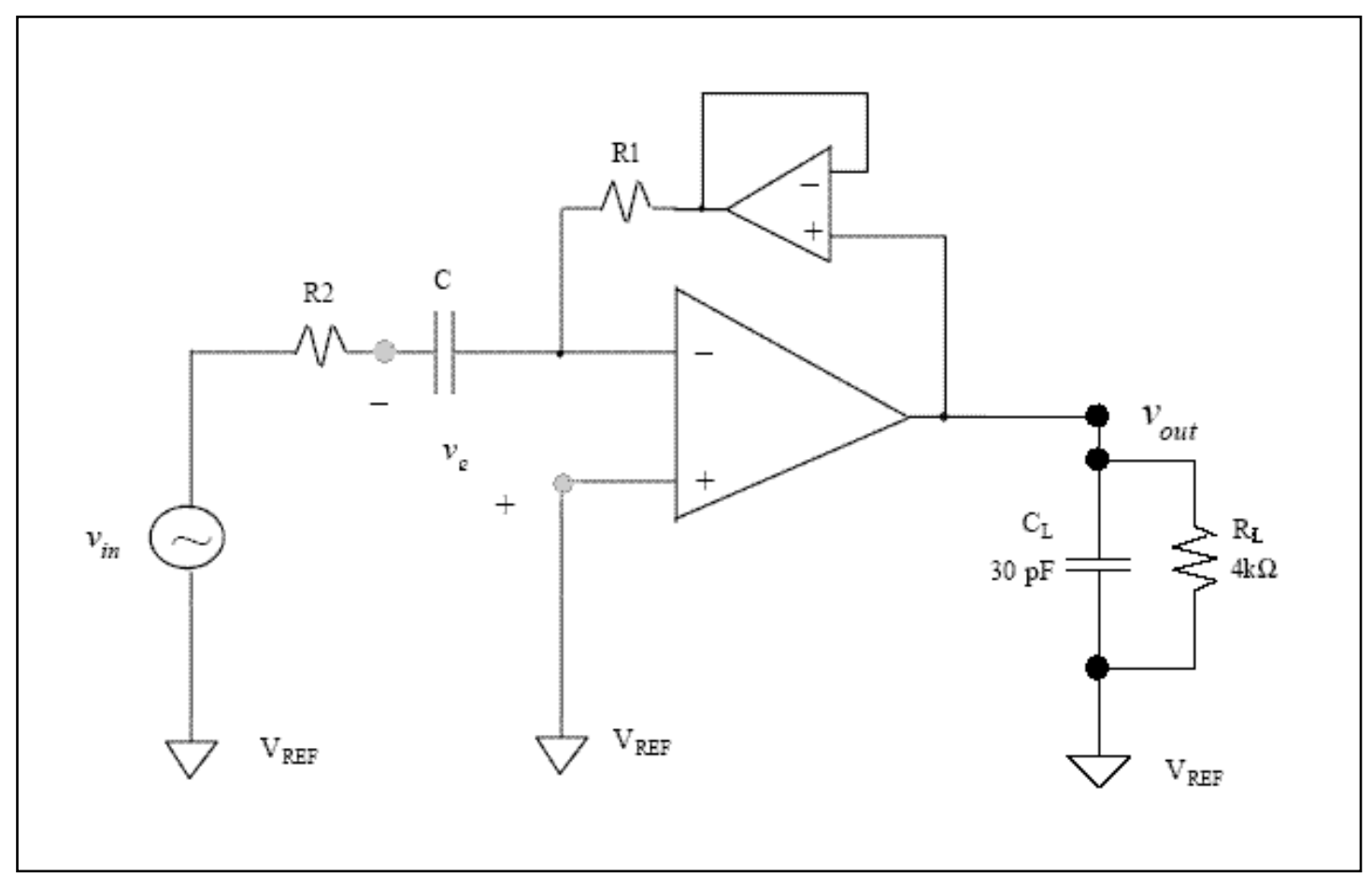

Figura 6.12. Capacitor $C$ introduzido para possibilitar a amplificação de $v_{e}$, eliminando o offset da medida realizada.

Logo, foram escolhidos os componentes mostrados na tabela 6.6, o que fornece uma relação $R_{1} \cdot C \cong 22>4,6$.

Tabela 6.6. Valores escolhidos para os componentes da figura 6.12.

\begin{tabular}{|c|c|c|}
\hline Componente & Valor & Unidade \\
\hline $\mathrm{R}_{1}$ & 100 & $\mathrm{k} \Omega$ \\
\hline $\mathrm{R}_{2}$ & 100 & $\mathrm{k} \Omega$ \\
\hline $\mathrm{C}$ & 200 & $\mu \mathrm{F}$ \\
\hline
\end{tabular}

A interpretação física deste resultado é a seguinte: ao ser introduzido o capacitor $C$, os sinais em baixas freqüências são bastante atenuados, pois $Z_{C}=\frac{1}{j \omega C}$. Portanto, o sinal que é efetivamente amplificado pelo ampOp (que aparece entre as entradas positiva e negativa) é bem menor do que o sinal introduzido pela fonte $v_{\text {in }}$. Conforme a freqüência do sinal aumenta, a impedância imposta pelo capacitor começa a diminuir, e a transferência do sinal entre os 
terminais do capacitor começa a aumentar até o momento em que a impedância do capacitor seja desprezível. Neste instante pode-se determinar o verdadeiro ganho do ampOp, sem influência do pólo introduzido pelo capacitor. É por esta razão que a constante " $R_{1} C$ " deve satisfazer a relação (6.1), pois quando $Z_{C}$ for desprezível, é necessário que o sinal ainda não tenha atingido a freqüência do primeiro pólo do ampOp. Caso a freqüência do sinal já seja maior ou igual à freqüência do primeiro pólo do circuito, não conseguiremos observar o ganho $D C$ de malha aberta.

A figura 6.13 apresenta duas curvas obtidas através de simulações. A primeira, chamada $A_{V}$, representa o ganho de malha aberta do amplificador sem 0 capacitor de acoplamento. A segunda, denominada $A_{V \_}$dif, representa o ganho de malha aberta do amplificador com o capacitor de acoplamento. Podemos observar que em baixas frequências a curva $A_{V}$ dif se aproxima da curva $A_{V}$, desde que a relação (6.1) seja respeitada. Isso é muito importante pois a curva que mediremos será $A_{V \_}$dif.

Os valores importantes do gráfico acima se encontram na tabela 6.7.

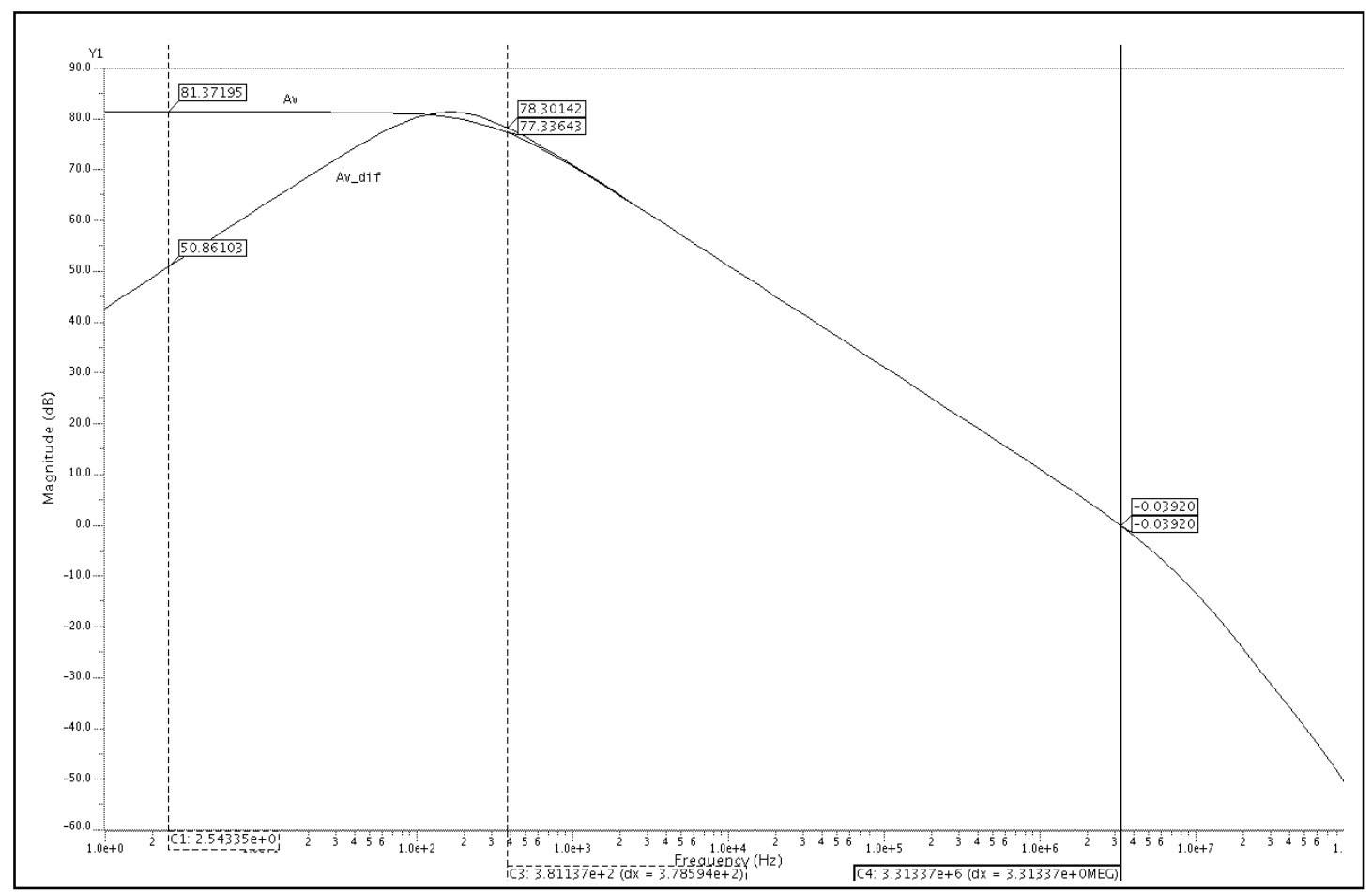

Figura 6.13. Simulação do módulo da resposta em freqüência do amplificador, utilizando um capacitor de $220 \mu \mathrm{F}$ de acoplamento na entrada negativa, conforme a figura 6.12.

Assim, obedecendo a condição (6.1), e fazendo uma varredura em freqüência da fonte $v_{\text {in }}$, obtém-se os valores de $v_{e}$ e $v_{\text {out }}$. O módulo de $\frac{v_{\text {out }}}{v_{e}}$ é 
apresentado na figura 6.14 e é igual ao módulo da resposta em freqüência do ampOp mais a influência do pólo causado pelo capacitor de entrada.

Tabela 6.7. Valores retirados da figura 6.13, caracterizando o módulo da resposta em freqüência do amplificador com capacitor de acoplamento na entrada negativa.

\begin{tabular}{|c|c|c|}
\hline Parâmetro & Valor & Unidade \\
\hline$A v$ & 81,3 & $\mathrm{~dB}$ \\
\hline$f p_{1}$ & 381 & $\mathrm{~Hz}$ \\
\hline$G B W$ & 3,3 & $\mathrm{MHz}$ \\
\hline
\end{tabular}

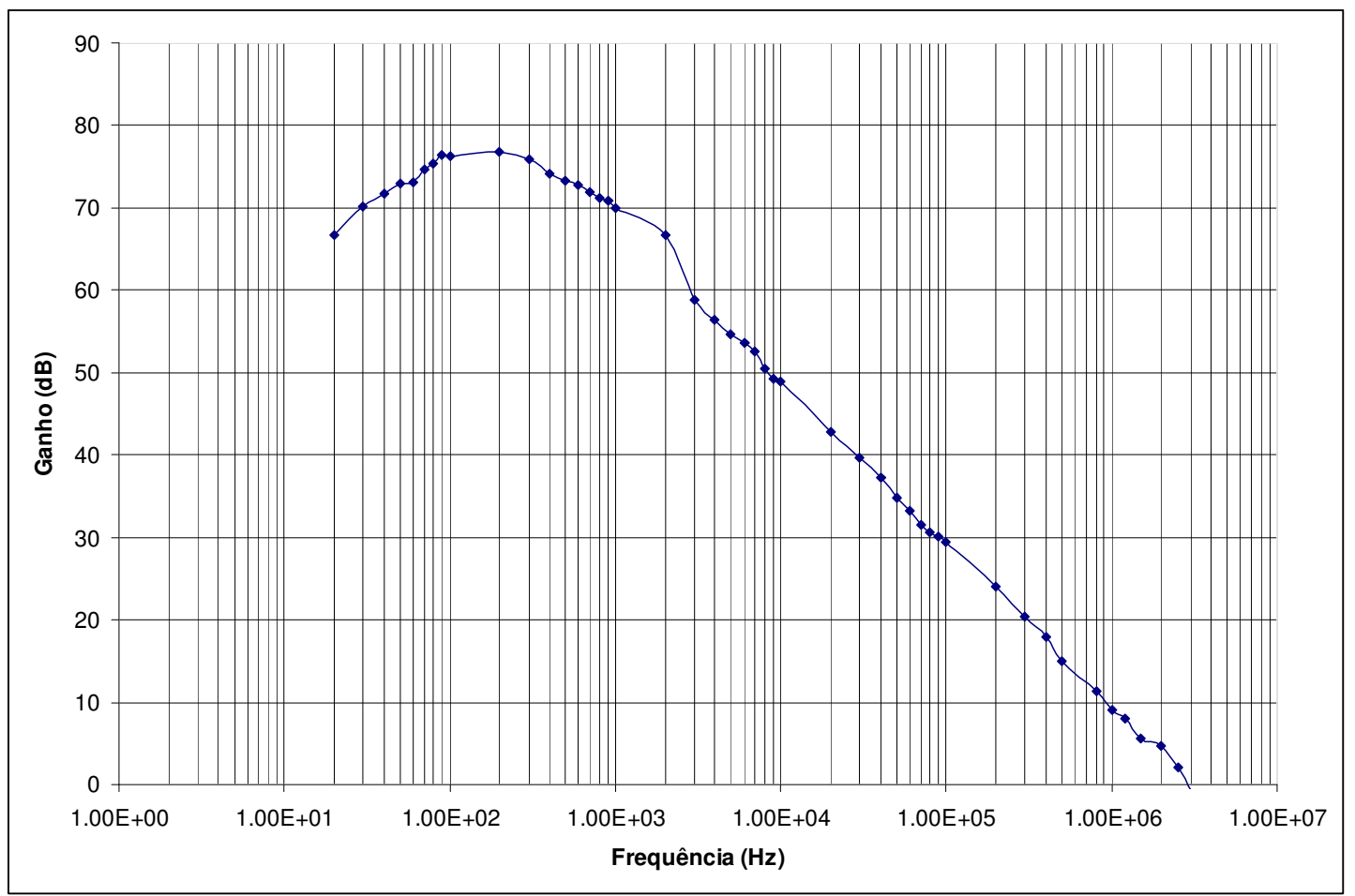

Figura 6.14. Módulo da resposta em frequiência do circuito da figura 6.12.

A tensão $v_{\text {in }}$ é um sinal senoidal de amplitude suficiente para que a saída do in-amp não sature e se encontre aproximadamente no meio da excursão entre as duas fontes de alimentação. É importante verificar, para cada medida, se essa condição é válida, pois em determinados momentos o sinal na saída do in-amp começa a oscilar em direção à $V_{D D}$ ou $V_{S S}$ e é ceifado. Isso algumas vezes é mascarado pelo ruído presente no sinal. 
A tabela 6.8 apresenta os principais pontos do gráfico acima.

Tabela 6.8. Medidas retiradas do gráfico da figura 6.14.

\begin{tabular}{|c|c|c|}
\hline Parâmetro & Medida & Unidade \\
\hline Ganho DC $\left(\mathrm{A}_{\mathrm{V}}\right)$ & 76,8 & $\mathrm{~dB}$ \\
\hline Primeiro Pólo $\left(\mathrm{f}_{\mathrm{P} 1}\right)$ & 400 & $\mathrm{~Hz}$ \\
\hline GBW & 2,9 & $\mathrm{MHz}$ \\
\hline
\end{tabular}

Apenas para verificação dos resultados, vamos calcular a posição do primeiro pólo. De acordo com (2.17),

$$
\begin{gathered}
G B W=A_{V} \cdot f p_{1} \Rightarrow f p_{1}=\frac{G B W}{A_{V}} \Rightarrow \text { sendo } A_{V}=76,8 d B s=6920[\mathrm{~V} / \mathrm{V}] \\
f p_{1}=\frac{G B W}{A_{V}}=\frac{2,9 M}{6920} \\
f p_{1}=419 \mathrm{~Hz}
\end{gathered}
$$

O resultado mostra que a frequência medida do primeiro pólo $(419 \mathrm{~Hz})$ está próxima da frequência simulada $(350 \mathrm{~Hz})$, o que ajuda a validar os resultados medidos.

\subsubsection{Medida CMRR e PSRR}

As medidas de CMRR e PSRR não foram realizadas pois, de acordo com os resultados de simulação, isso não é possível tanto para freqüências baixas com para médias. Apenas como ilustração, e utilizando os valores das tabelas 5.2 e 5.3, o valor de $C M R R$ em baixas freqüências é $137 \mathrm{dBs}$. Assim, para ter sinal na saída do ampOp de apenas $1 \mathrm{mV}$, deveremos ter como entrada um sinal de amplitude maior do que $7.000 V$ ! Fazendo o raciocínio inverso, supondo que a fonte de entrada introduza um sinal de $1 \mathrm{~V}$ de modo comum, teremos na saída do ampOp um sinal de $0,14 \mu V$, o que torna a medida impraticável. Consideração semelhante se aplica à medida de $P S R R$.

\subsection{Caracterização Transisente}

\subsubsection{Medida do Slew Rate}

A figura 6.15 apresenta o testbench utilizado para medição do slew rate do circuito. 


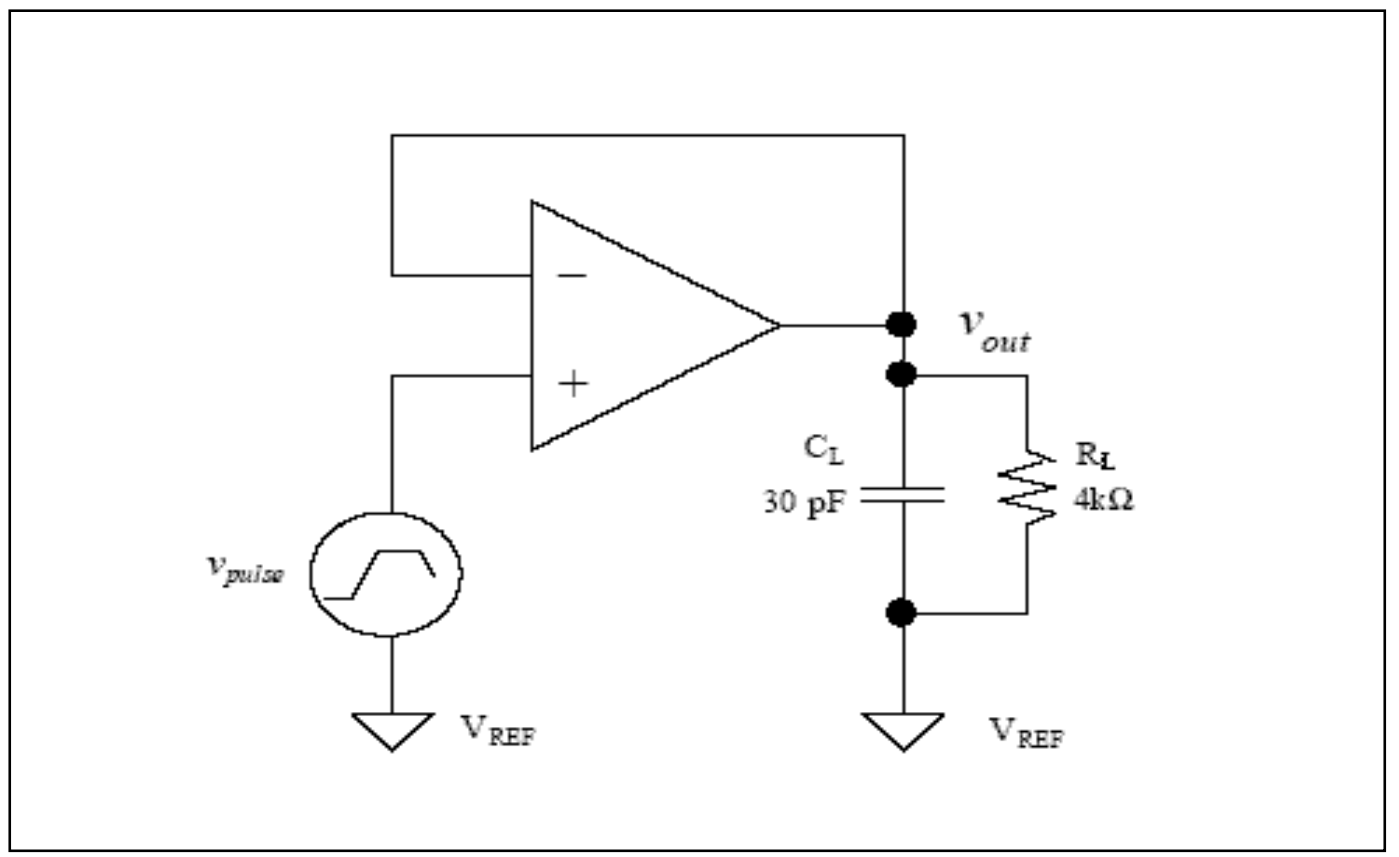

Figura 6.15. Testbench utilizado para medida de slew rate e margem de fase

A figura 6.16 ilustra a forma de onda esperada na saída e como deve ser medido o SR. Dados do testbench:

- $\mathrm{V}_{\text {in pp }}: 1 \mathrm{~V}$, onda quadrada;

- Freqüência: $640 \mathrm{kHz}$

- $\mathrm{V}_{\text {médio: }}: 1,4 \mathrm{~V}$.

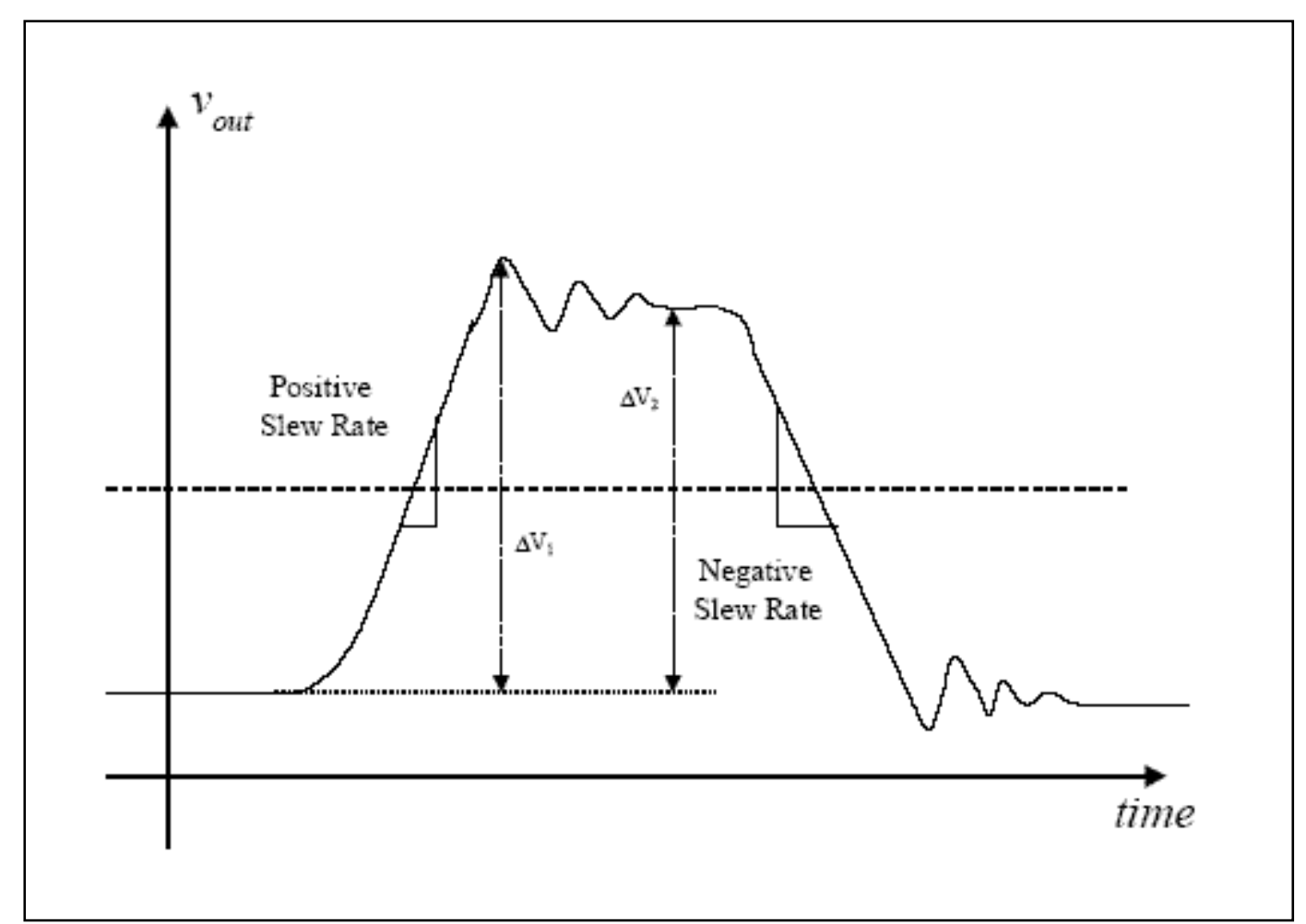

Figura 6.16. Resposta transiente, ilustrativa, do circuito à um pulso quadrado na entrada. 
Cálculo do SR positivo:

$$
S R=\frac{\Delta V}{\Delta t}=\frac{185 \mathrm{mV}}{72 \mathrm{~ns}}=2,57 \mathrm{~V} / \mu \mathrm{s}
$$

Cálculo do SR negativo:

$$
S R=\frac{\Delta V}{\Delta t}=\frac{-150 m V}{72 n s}=-2,08 \mathrm{~V} / \mu \mathrm{s}
$$

\subsubsection{Medida da Margem de Fase}

A margem de fase do circuito deve ser medida através da resposta transiente do ampOp à um degrau na entrada. Porém, diferentemente da caracterização de slew rate, onde é importante que o sinal tenha uma "grande" amplitude (da ordem de Volt), pois se trata de uma característica de grandes sinais, a caracterização da margem de fase se dá através de um pulso de pequena amplitude na entrada (da ordem de miliVolt), de forma que o circuito permaneça operando sob a suposição de pequenos sinais, para que suas características de polarização não se alterem.

Para calcular a margem de fase do circuito será medido o overshoot do sinal de saída. Intuitivamente podemos perceber que quanto maior o overshoot menor será a margem de fase, pois o sistema, neste caso, tende a oscilar. Entretanto, mais rápida será a resposta do sistema. Por outro lado, quanto menor o overshoot, maior será a margem de fase pois o sistema é mais estável. Observemos que com uma margem de fase pequena temos um circuito com respostas mais rápidas.

O mesmo testbench utilizado para medida de slew rate será utilizado para verificação da margem de fase, apenas modificando a amplitude do sinal de entrada. Dados do testbench:

- $\mathrm{V}_{\text {in pp }}: 346,1 \mathrm{mV}$, onda quadrada;

- Freqüência: $350,8 \mathrm{kHz}$

- $\mathrm{V}_{\text {médio: }}$ 1,411 V.

Cálculo do fator de amortecimento $\xi$ [8:

- Tensão de pico ( $\Delta V_{1}$ na figura 6.16$)$ do overshoot: 1,501 V;

- Valor final de tensão ( $\Delta V_{2}$ na figura 6.16): 1,499 V.

$$
\begin{aligned}
\frac{(\text { valor de pico })-(\text { valor final })}{\text { (valor final })} & =e^{\frac{-\pi \cdot \xi}{\sqrt{1-\xi^{2}}}} \Rightarrow \frac{1,501-1,499}{1,499}=e^{\frac{-\pi \cdot \xi}{\sqrt{1-\xi^{2}}}} \\
\xi & =0,9034
\end{aligned}
$$


Cálculo da margem de fase:

$$
\Phi_{M}=\arctan \left(2 \cdot \xi \cdot \sqrt{\frac{1}{\sqrt{4 \cdot \xi^{4}+1}-2 \cdot \xi^{2}}}\right)
$$

substituindo o valor de $\xi$ calculado,

$$
\Phi_{M}=73,6^{\circ}
$$




\section{Capítulo 7}

\section{Conclusões}

Vamos iniciar este capítulo apresentando uma comparação entre os resultados projetados do capítulo 3 , os resultados simulados do capítulo 5 e os resultados medidos do capítulo 6. A tabela 7.1 consolida esses dados.

Tabela 7.1. Comparação entre os resultados projetados, simulados e medidos do ampOp.

\begin{tabular}{|c|c|c|c|c|}
\hline Parâmetro & Projeto & Simulação & Medida & Unidade \\
\hline Offset & ------- & ------- & $-1,7$ & $\mathrm{mV}$ \\
\hline$I C M R$ & ------- & {$[0,0 ; 2,3]$} & {$[0,1 ; 2,2]$} & V \\
\hline Excursão Saída & ------- & {$[0,06 ; 2,74]$} & {$[0,0 ; 2,6]$} & V \\
\hline$A_{V}$ & ------- & 81,4 & 76,8 & $\mathrm{~dB}$ \\
\hline$G B W$ & 3,5 & 3,3 & 2,9 & $\mathrm{MHz}$ \\
\hline$f p_{1}$ & ------- & 310 & 419 & $\mathrm{~Hz}$ \\
\hline$M F$ & 60 & 62 & 73,6 & $\circ$ \\
\hline CMRR (B.F.)* & ------ & 137 & ------- & $\mathrm{dB}$ \\
\hline CMRR (GBW) & ------- & 53 & ------- & $d B$ \\
\hline $\operatorname{PSRR}(\text { B.F. })^{*}$ & ------ & 118,6 & ------ & $d B$ \\
\hline PSRR (GBW) & ------- & 29,8 & ------ & $\mathrm{dB}$ \\
\hline $\begin{array}{c}\text { Slew Rate } \\
\text { (pos.) }\end{array}$ & 2 & 2,86 & 2,57 & $V / \mu s$ \\
\hline $\begin{array}{c}\text { Slew Rate } \\
\text { (neg.)** }\end{array}$ & -2 & $-1,75$ & $-2,08$ & $V / \mu s$ \\
\hline
\end{tabular}

${ }^{*}$ B.F. = Baixas Freqüências.

** Admitiu-se, para projeto, que o Slew Rate negativo seria igual, em módulo, ao Slew Rate positivo.

A tabela mostra que os valores medidos estão relativamente próximos dos valores simulados. Obviamente que a comparação desses resultados com os 
resultados de projeto devem apresentar uma diferença maior pois, para o projeto, foram utilizadas expressões simplificadas, conforme explicado no decorrer do texto.

Nota-se uma pequena variação entre os ganhos $A_{V}$ medido e simulado, o que pode ser justificado por duas razões: a dificuldade de se realizar as medidas de ganho com maior precisão e a dependência natural desse parâmetro com as variações inerentes ao processo de fabricação, as variações de temperatura e polarização.

A diferença observada na banda de ganho unitário, GBW, pode ser atribuída às eventuais capacitâncias parasitas existentes no circuito, e ao próprio fato do ganho $A_{V}$ ser menor do que o ganho simulado.

A margem de fase, $M F$, apresenta uma variação considerável no sentido de estabilizar ainda mais o circuito, o que pode se justificar pela diminuição de $G B W$, e pela frequência do segundo pólo praticamente não ter mudado, ou seja, GBW diminuiu proporcionalmente mais do que o segundo pólo.

Já o parâmetro $S R$ (positivo) medido se aproxima bastante dos resultados simulados, apresentando uma pequena diminuição de seu valor, o que pode ser justificado, mais uma vez, por capacitâncias parasitas que não foram consideradas durante o projeto. Porém, o $S R$ (negativo) medido apresentou um valor maior, em módulo, do que o resultado simulado, o que, apesar de representar uma melhoria na performance do circuito fabricado, não era um resultado esperado. Um ponto importante a ser ressaltado é a diferença existente entre o $S R$ (positivo) e o $S R$ (negativo). Durante a fase de projeto foi assumido que os dois valores seriam iguais, o que não se observou durante a simulação e as medidas. Uma explicação para essa diferença pode estar na diferença de capacidade de fornecer e absorver corrente das fontes formadas por M11-M12 (fornecedora de corrente) e M7-M8 (absorvedora de corrente).

Os demais parâmetros, CMRR e PSRR, não foram medidos, por razões práticas, sendo apenas simulados. Seus valores, porém, são bastante elevados, o que significa que o circuito apresenta uma boa rejeição tanto à sinais na fonte de alimentação quanto à variações na tensão de modo comum da entrada.

Após o projeto do amplificador folded cascode deu-se início à implementação do software de auxílio ao projeto, que foi escrito tendo como referência este primeiro projeto do ampOp. Os resultados obtidos através do software eram comparados com os resultados projetados anteriormente, até que os valores de $\mathrm{W} / \mathrm{L}$ dos transistores fossem relativamente próximos. Concluída esta etapa, o software foi utilizado para reprojetar o ampOp de forma a atender novas especificações, como por exemplo 
$G B W=20 \mathrm{MHz}, M F=80^{\circ}$, etc., sendo que esses novos resultados foram validados através de simulações. O reprojeto foi realizado seguindo a máquina de estados definida no capítulo 4, valendo a pena ressaltar alguns pontos importantes:

1. Como não há restrição quanto aos tamanhos mínimos de comprimento de canal, ou máximo de largura de canal, alguns resultados de reprojeto forneceram $L<L_{\text {MiN }}$, onde $L_{\text {MíN }}$ é o menor comprimento de canal permitido na tecnologia. Ou então alguns transistores foram reprojetados com um valor de $W$ bastante elevado, aumentando muito a área ocupada do chip;

2. Não foi introduzido no software nenhum algoritmo que otimizasse alguma característica do circuito, como consumo máximo, área máxima, etc;

3. Outras formas de reprojetar o ampOp devem ser estudadas e implementadas, de forma a se escolher a que atenda melhor às necessidades do projetista.

Neste trabalho se mostrou viável a implementação de um software para projetos de cores analógicos. As maiores dificuldades para isso foram identificadas e algumas soluções foram propostas, como por exemplo a utilização do software em conjunto com um simulador elétrico, de forma a se extrair parâmetros de pequenos sinais com a precisão necessária; a utilização da técnica de orientação à objetos na implementação deste software, de forma a torná-lo modular, flexível, e possibilitando acrescentar novos blocos analógicos. Há também a questão do procedimento utilizado no reprojeto realizado pelo software: como dito, é possível escolher procedimentos diferentes para reprojetar um bloco analógico, cada um com suas vantagens e desvantagens. O escolhido neste trabalho tem a vantagem de possibilitar ao projetista um controle maior sobre as variações dos parâmetros do circuito.

Espera-se que futuramente outros projetistas possam dar continuidade à este trabalho, introduzindo novos cores analógicos. Assim o tempo de projeto e os erros podem ser minimizados, melhores resultados podem ser obtidos e, também, tornase possível avaliar rapidamente as características de um determinado core, verificando se atende às especificações ou se outra solução é necessária. Em um momento em que as janelas para o lançamento de novos produtos no mercado se tornam cada vez mais estreitas, a utilização de softwares para projetos de cores analógicos pode permitir que uma empresa chegue ao mercado algumas semanas antes da concorrência, tornando-se líder no seu segmento, o que é a diferença entre sucesso e fracasso. 


\section{Referências}

[1] HOGERVORST, R., TERO, J.P., ESCHAUZIER, R.G.H., HUIJSING, J.H., "A Compact Power-Efficient 3V CMOS Rail-to-Rail Input/Output Operational Amplifier for VLSI Cell Libraries”, IEEE Journal of Solid-State Circuits, v. 29, p. 1505-1512, December, 1994.

[2] LANGEN, K., HUIJSING J.H., "Compact Low-Voltage Power-Efficient Operational Amplifier Cells for VLSI", IEEE Journal of Solid-State Circuits, v. 33, n.10, p. 1482-1496, October, 1998.

[3] STEYAERT, M., SANSEN, W., HUIJSING J.H., PLASSCHE, R. "Opamp Design towards Maximum Gain-Bandwidth". In: Analog circuit design. Operational amplifiers, analog to digital converters, analog computer aided design, $1^{\text {st }}$ ed, Delft: Kluwer Academic Publishers, March 1993. p. 63-85.

[4] CRAIN, E., PERROTT, M.H., "A numerical Design Approach for High-Speed, Differential, Resistor-Loaded, CMOS Amplifiers". In: IEEE INTERNATIONAL SYMPOSIUM ON CIRCUITS AND SYSTEMS, 2004, Vancouver, Canada.

[5] PARDOEN, M., "A Simple and Pretty Accurate MOS Model for Hand Calculations", Internet, http://rfsilicon.com/home/modeling/mos/mosmodel.PDF, 1999.

[6] GRAY, P.R., MEYER, R.G., "MOS Operational Amplifier Design - A Tutorial Overview," IEEE Journal Solid-State Circuits, v. SC-17, n. 6, p. 969-983, December, 1982.

[7] CAPELLI, A., "Amplificadores Operacionais Aplicados à Indústria", Saber Eletrônica, v. 37, n. 324, p.4-14, Julho, 2001.

[8] ALLEN, P.E., HOLBERG, D.R., "CMOS Analog Circuit Design", $2^{\text {nd }}$ ed, New York: Oxford University Press, 2002.

[9] GRAY, P.R., HURST, P.J., LEWIS, S.H., MEYER, R. G., "Analysis and Design of Analog Integrated Circuits", $4^{\text {th }}$ ed, New York: John Wiley \& Sons, 2001. 
[10] RAZAVI, B., "Design of Analog CMOS Integrated Circuits", $1^{\text {st }}$ ed, New York: McGraw-Hill, 2001.

[11] GREgORIAN, R., TEMES, G.C., "Analog Integrated Circuits for Signal Processing", $1^{\text {st }}$ ed, USA: John Wiley \& Sons, 1986.

[12] HASTINGS, A., "The Art of Analog Layout", $1^{\text {st }}$ ed, Prentice Hall, 2000.

[13] CHANG, J., ABIDI, A.A., VISWANATHAN, C.R., "Flicker Noise in CMOS Transistors from Subthreshold to Strong Inversion at Various Temperatures", IEEE Transactions on Electron Devices, v. 41, n. 11, p. 1965-1971, November, 1994.

[14] SEDRA, A. S., SMITH, K. C., "Microelectronic Circuits", $4^{\mathrm{TH}}$ ed, New York: Oxford University Press, 1998.

[15] Li, H., "Characterization of a Two-Stage Opamp", Internet, www.mrc.uidaho.edu/mrc/people/harry/ee415.

[16] KESTER, W., JUNG, W. HASTINGS, A., "Section 2-1: Instrumentation Amplifiers". In: Operational Amplifiers, National Devices, 2002. p. 2.1 - 2.29.

[17] PELGROM M., DUINMAJER A., WELBERS A., "Matching properties of MOS transistors", IEEE J. Solid-State Circuits, v. 24, n. 5, p. 1433-1439, 1989.

[18] KINGET P., "Device Mismatch and Tradeoffs in the Design of Analog Circuits", IEEE J. Solid-State Circuits, v. 40, n. 6, p. 1212-1224, June 2005.

[19] AUSTRIAMICROSYSTEMS, "0.35 $\mu \mathrm{m}$ CMOS C35 process parameters", Eng182, rev. 2.0, March 2003.

[20] AUSTRIAMICROSYSTEMS, “0.35 $\mu \mathrm{m}$ CMOS C35 Design Rules”, Eng-183, rev. 3.0, August 2003. 
[21] AUSTRIAMICROSYSTEMS, “0.35 $\mu \mathrm{m}$ CMOS C35 Matching Parameters”, Eng228, rev. 1.0, March 2003.

[22] LACERDA F., "Projeto de um Amplificador Operacional CMOS de Baixa Tensão do tipo Rail-to-Rail". Campinas: Unicamp, 2001, 147p. 


\section{Apêndice A - Rotinas do Software}

Nesta seção serão apresentadas as rotinas principais do software de auxílio ao projeto. Inicialmente será apresentado o arquivo de cabeçalho (header) que define a estrutura do amplificador e os objetos inputstage, biasstage, etc. que foram apresentados no capítulo 4. Depois é apresentada a rotina completa do arquivo design.c, que coném a rotina principal do programa e mostra a sequência utilizada para o projeto do ampOp. 


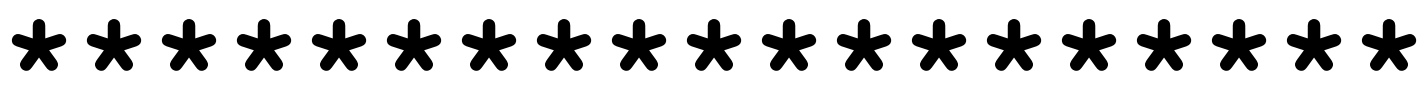

\section{DESIGN . H}

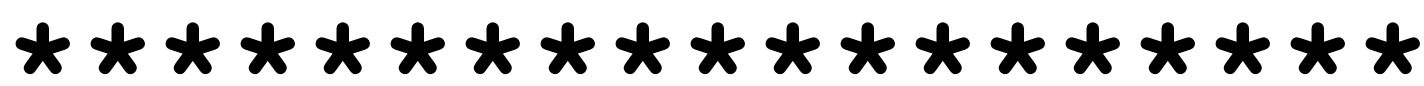




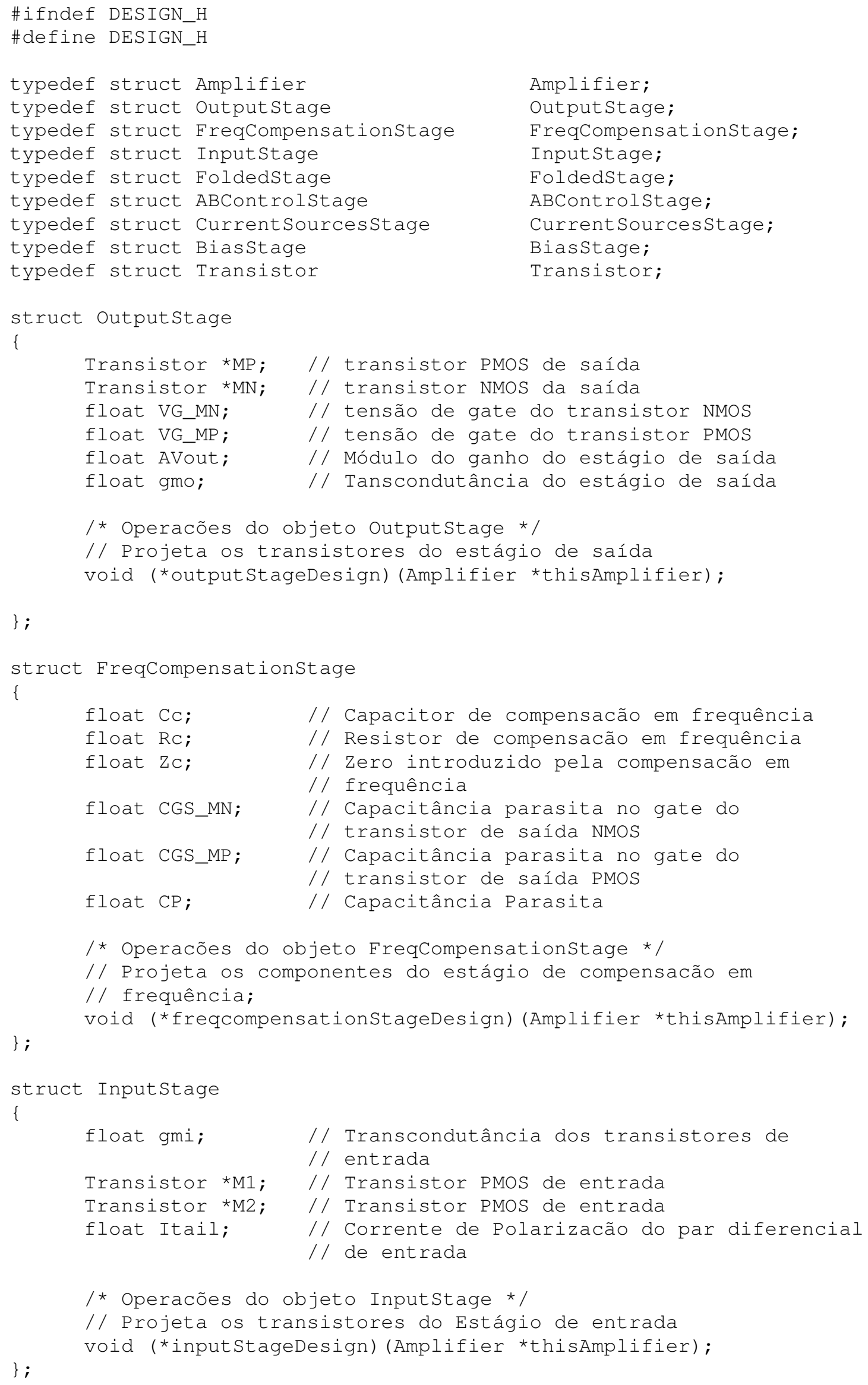




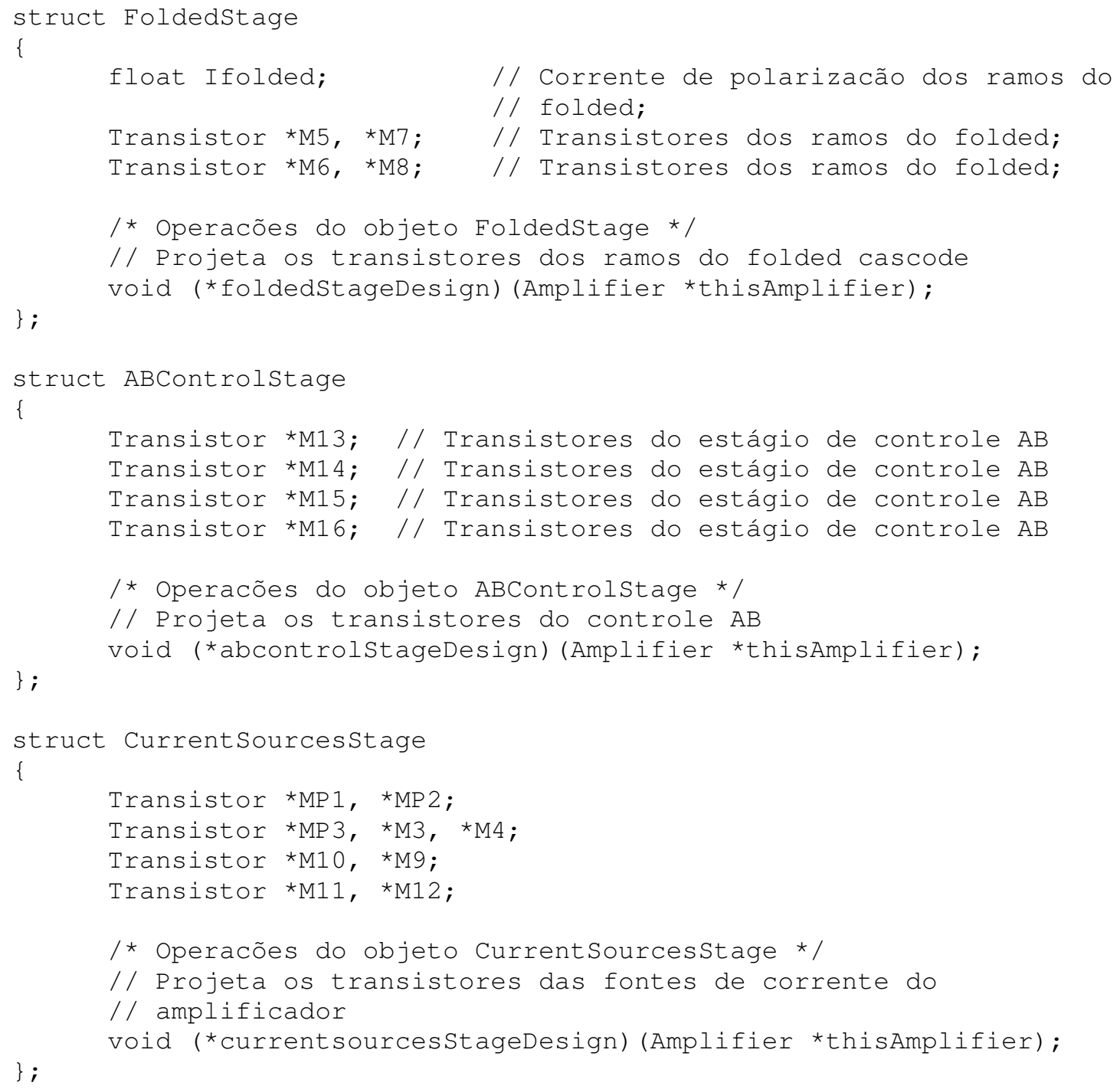


\} ;

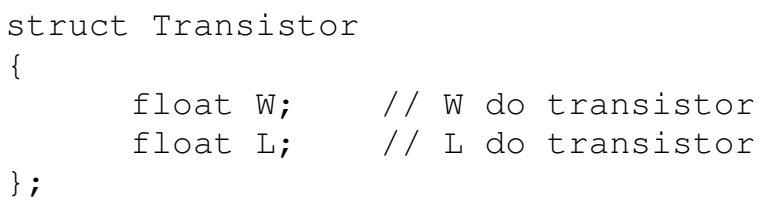

float gds_M7; // Condutância de M7, extraída do arquivo

// de saída da simulacão, utilizada para

// calcular GBW Full

float gds_M8; // Condutância de M8, extraída do arquivo

// de saída da simulacão, utilizada para

// calcular GBW Full

float gm_M1; // Transcondutância de M1, extraída do

// arquivo de saída da simulacão,

// utilizada para calcular GBW Full

float gm_M8; // Transcondutância de M8, extraída do

// arquivo de saída da simulacão,

// utilizada para calcular GBW Full

float gm_MP; // Transcondutância de MP, extraída do

// arquivo de saída da simulacão,

// utilizada para calcular GBW Full

float gm_MN; // Transcondutância de MN, extraída do

// arquivo de saída da simulacão,

// utilizada para calcular GBW Full

float phase_margin_sim; // Margem de Fase extraída da simulacão // do circuito projetado

float gbw_sim; // GBW extraída da simulacão do // circuito projetado

float dc_gain_sim;

// Ganho DC extraído da simulacão do // circuito projetado

/* Estágio de Saída */

Outputstage *output_stage;

/ Estágio de Compensacão em Frequência */

FreqCompensationstage *freqcompensation_stage; 


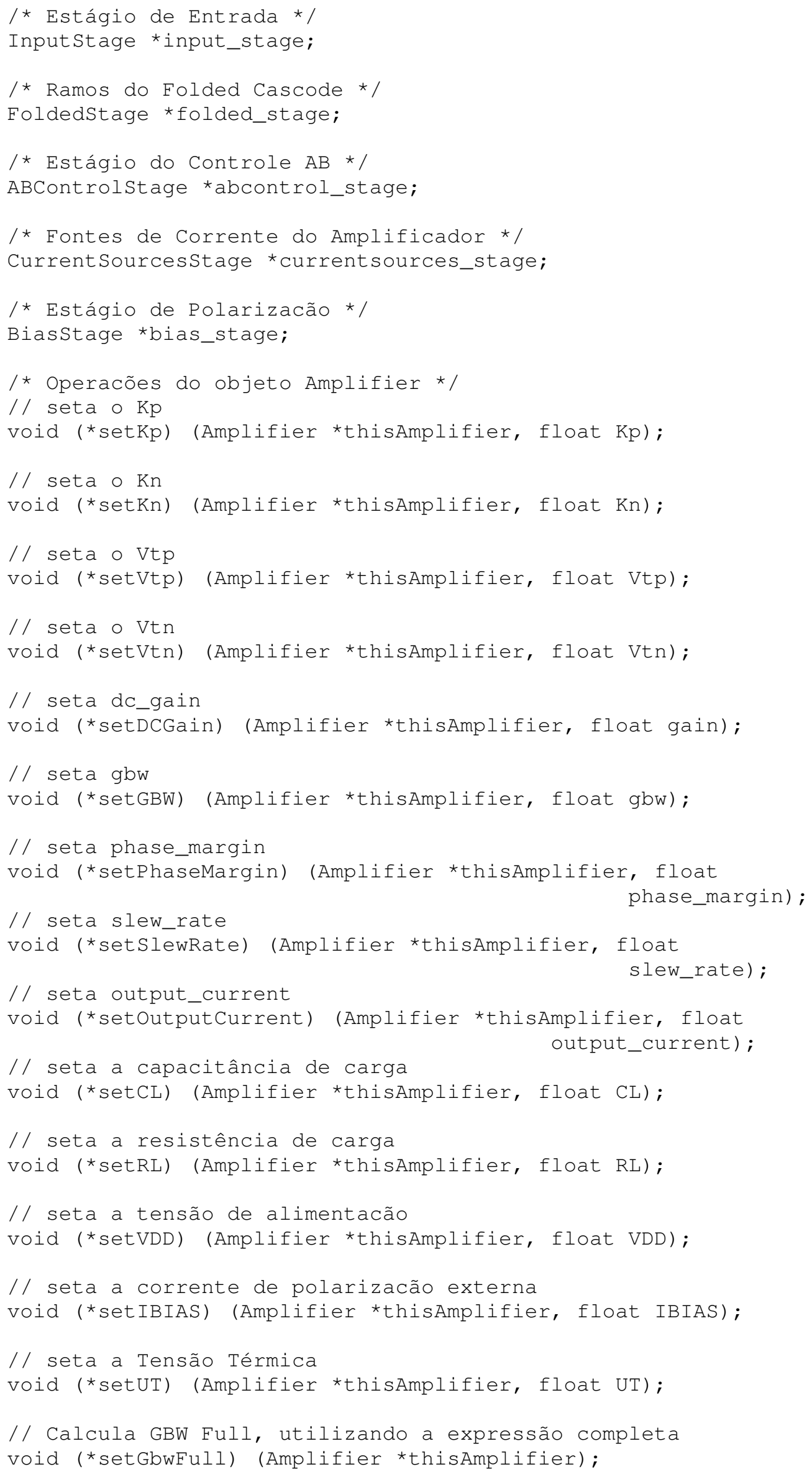




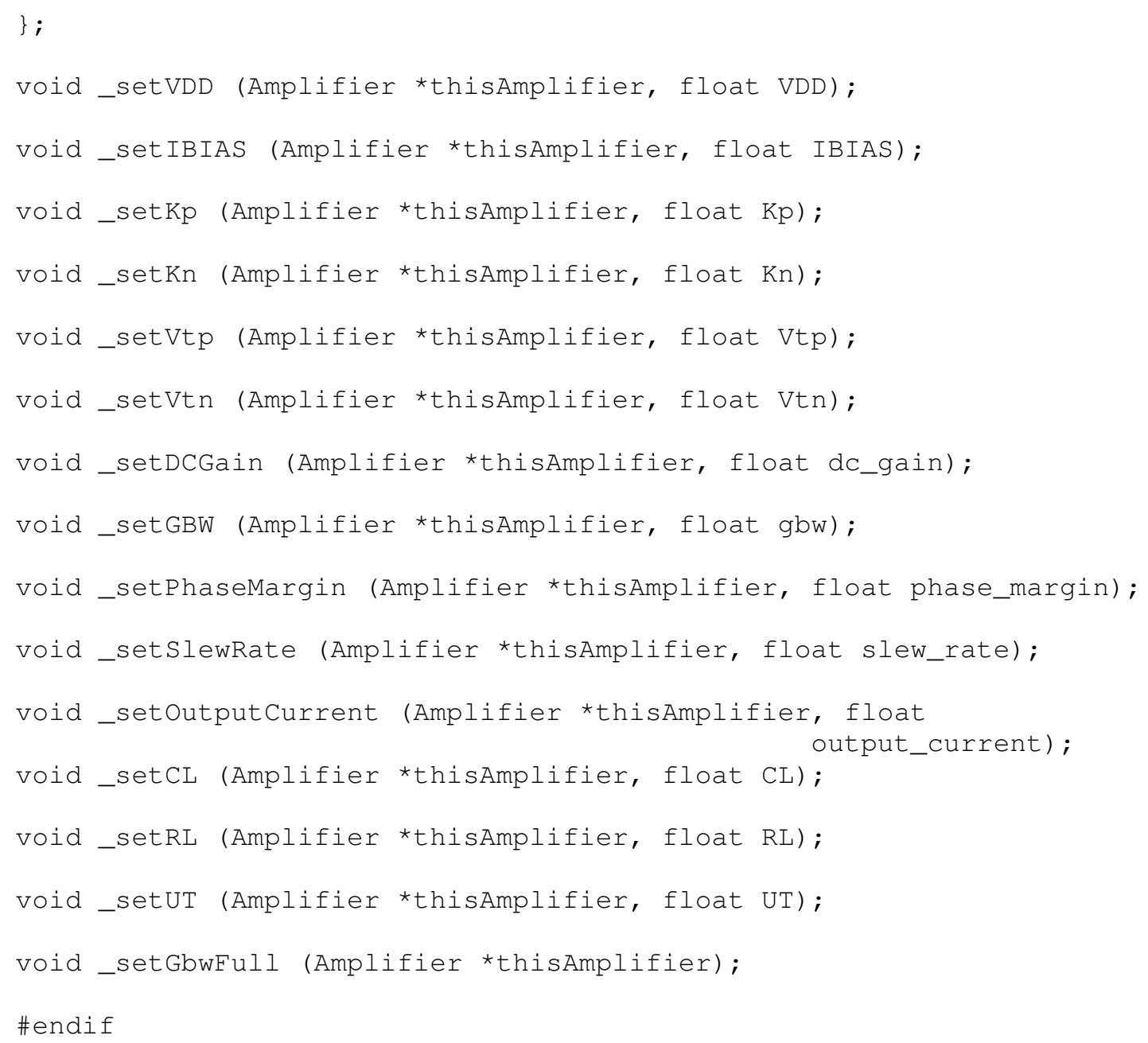




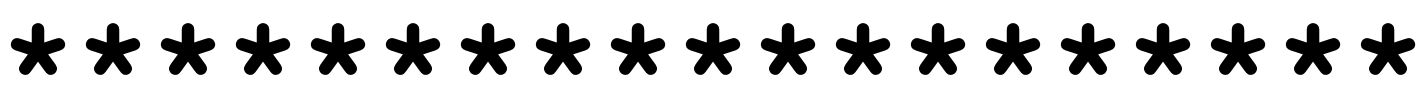

$$
\text { DESIGN.C }
$$

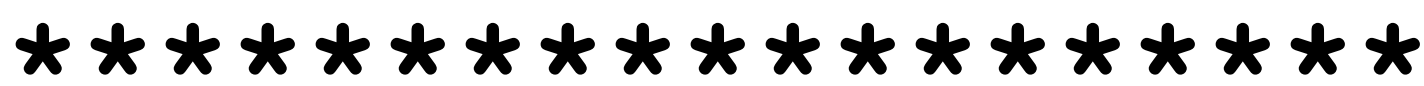




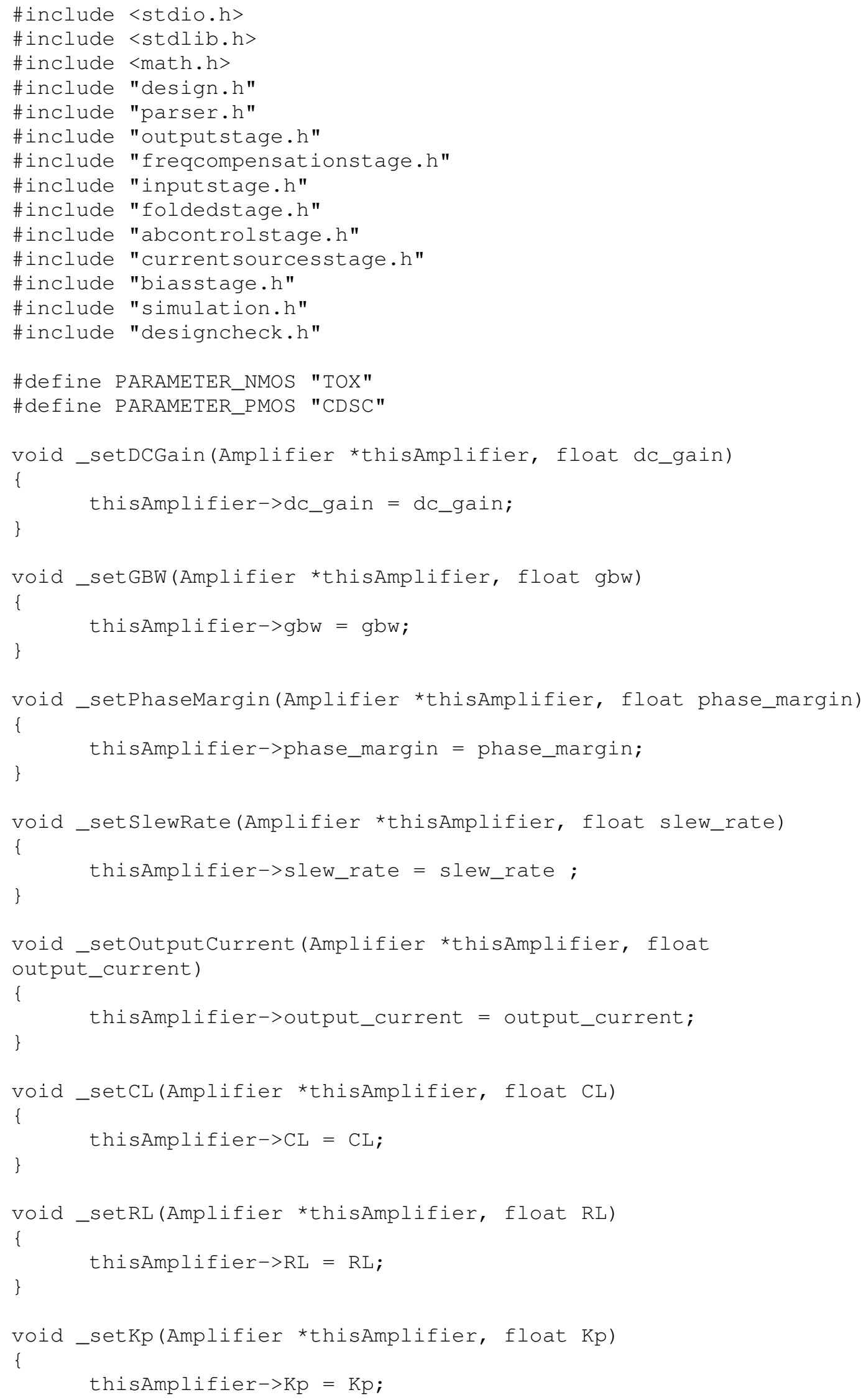




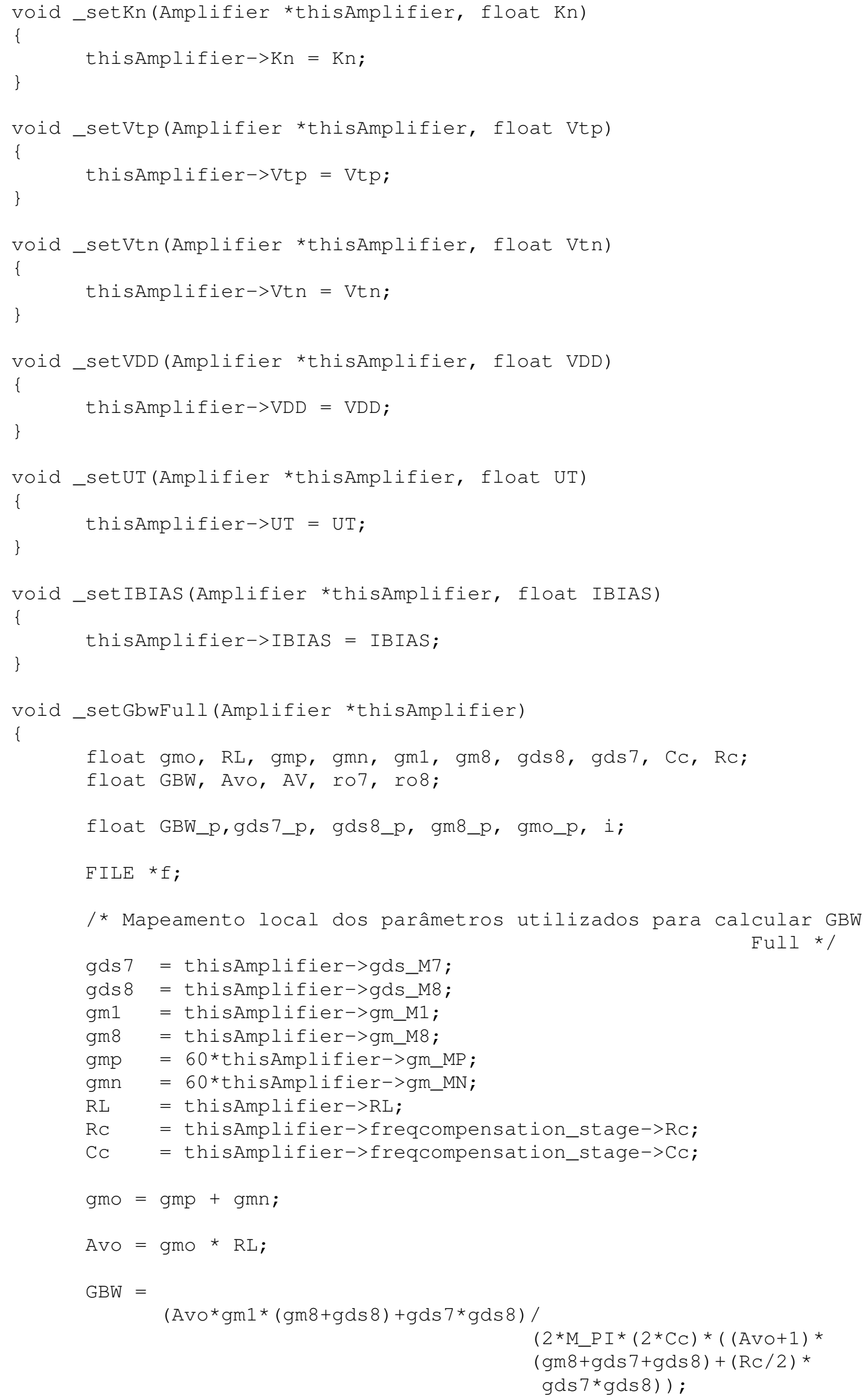




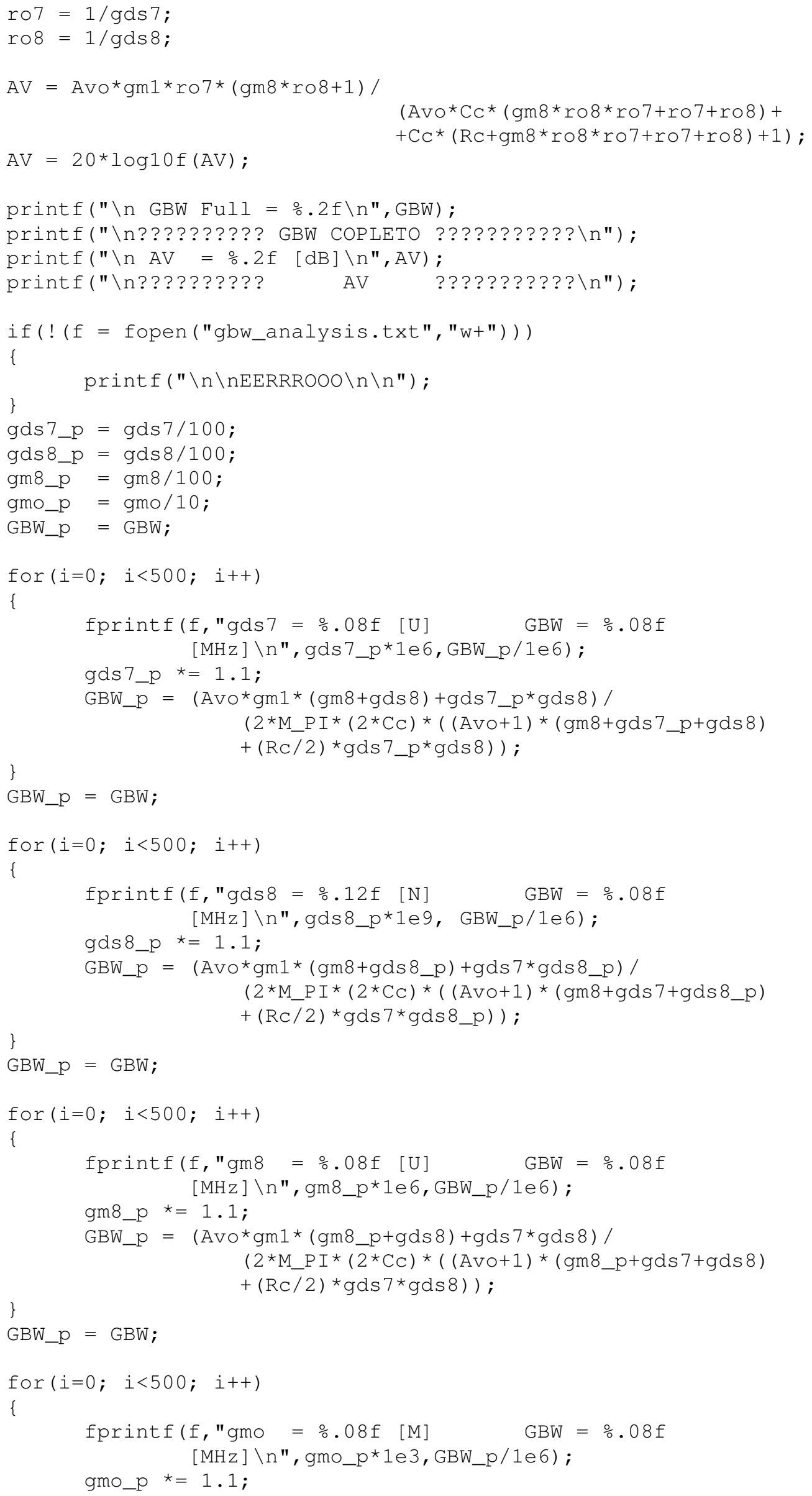




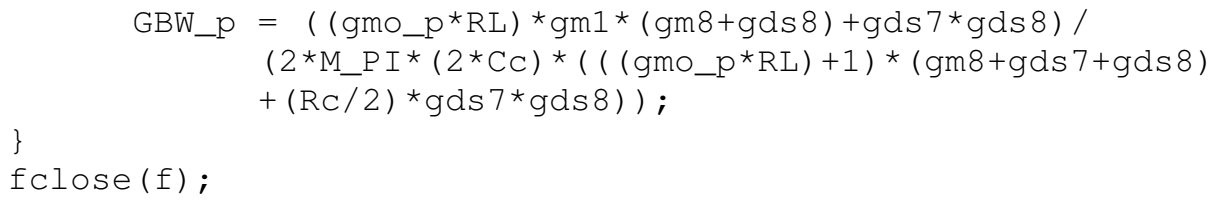


/* seta as tensões de threshold dos transistores PMOS e NMOS * / thisAmplifier->setVtp (thisAmplifier, 0.6915);

thisAmplifier->setVtn(thisAmplifier, 0.4975);

/* Mapeamento dos métodos de Projeto dos Ramos do Amplificador Folded Cascode */

thisAmplifier->folded_stage->foldedStageDesign = _foldedStageDesign;

/* Mapeamento métodos de Projeto do Estágio de Controle AB */ thisAmplifier->abcontrol_stage->abcontrolstageDesign = _abcontrolstageDesign;

/* Mapeamento dos métodos de Projeto das Fontes de Corrente do Amplificador */

thisAmplifier->currentsources_stage->currentsourcesstageDesign =_currentsourcesstageDesign;

/* Mapeamento métodos de Projeto do Estágio de Polarizacão */ thisAmplifier->bias_stage->biasStageDesign = _biasstageDesign; 
int main (void)

\{

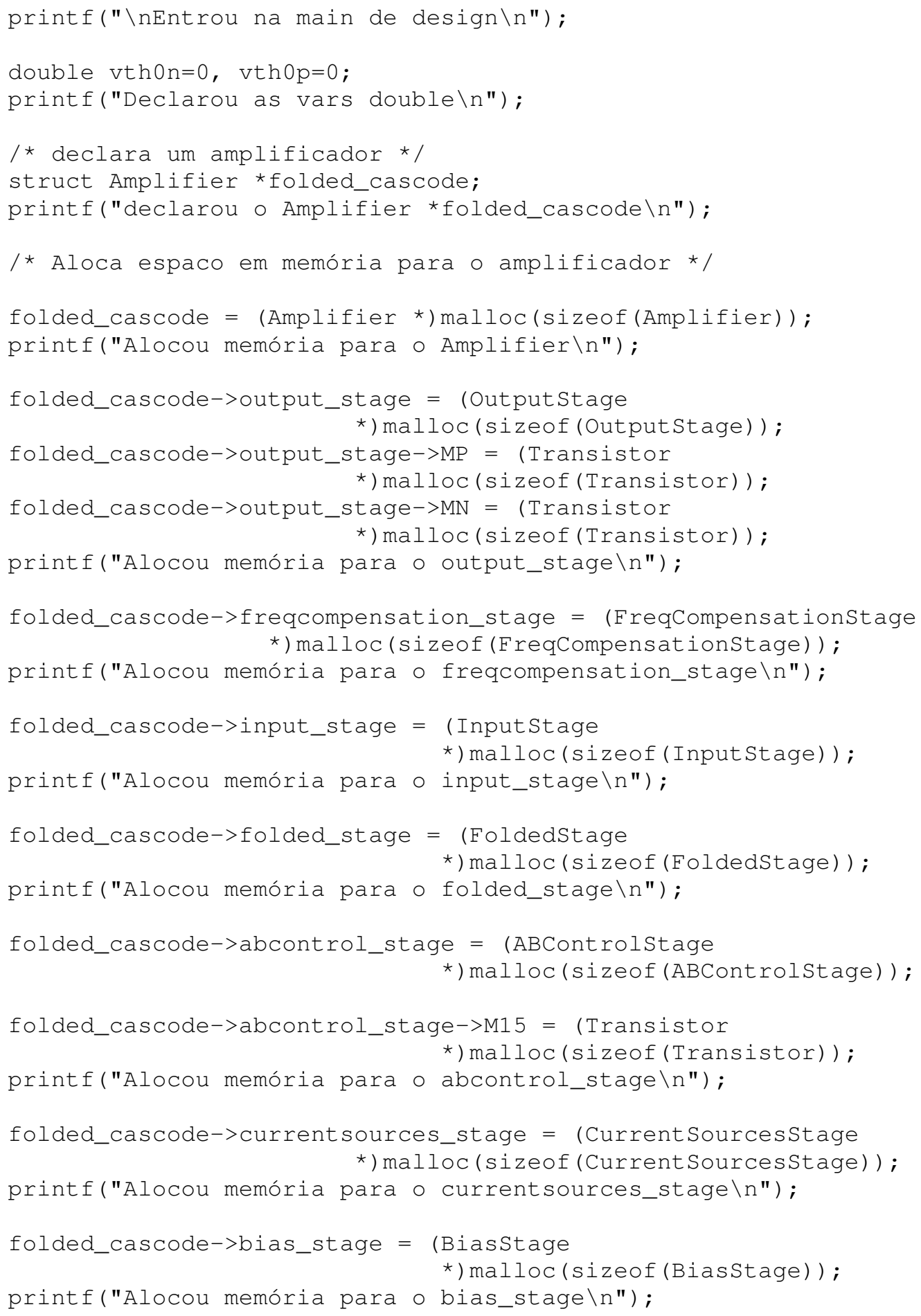




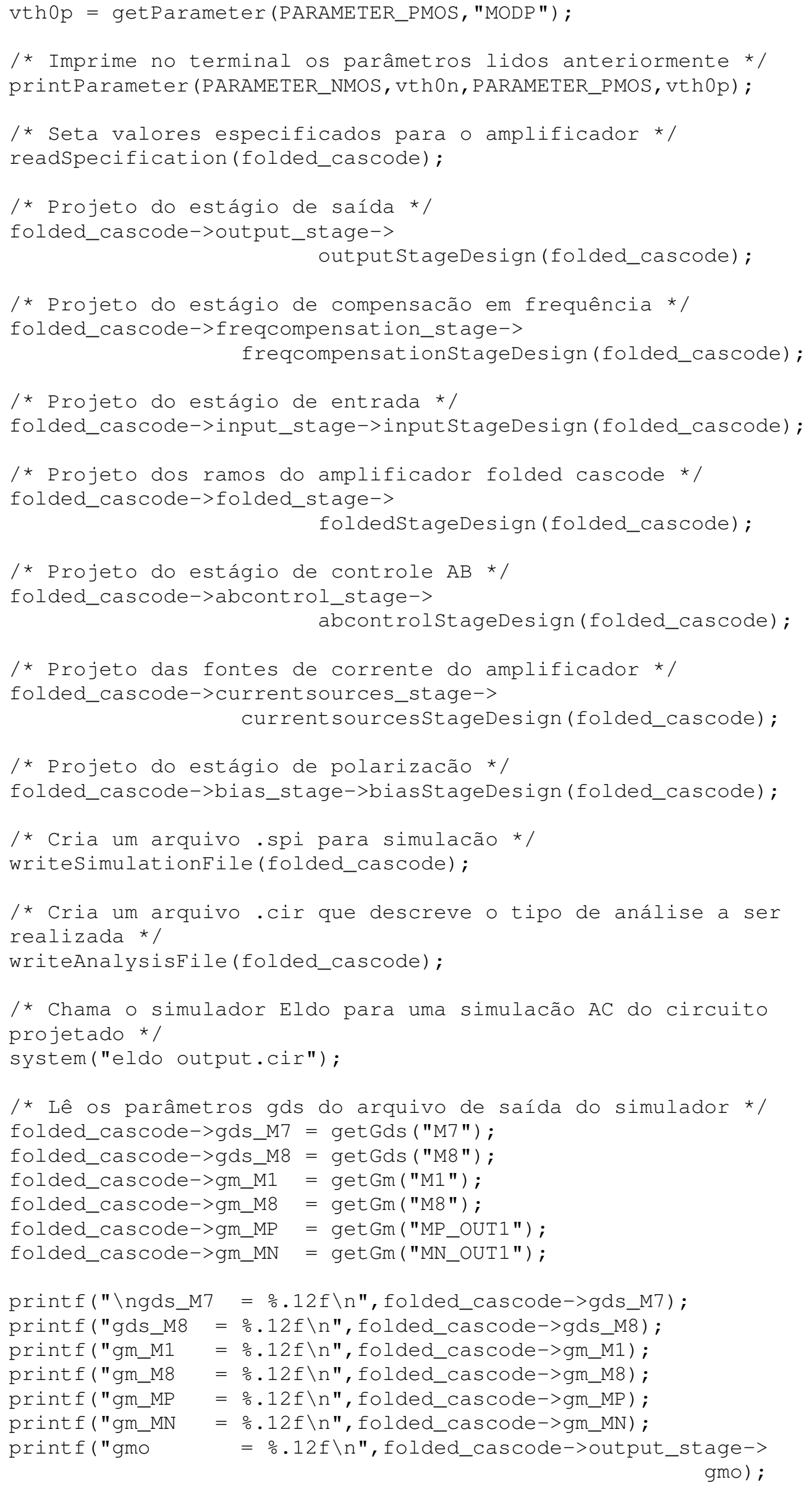




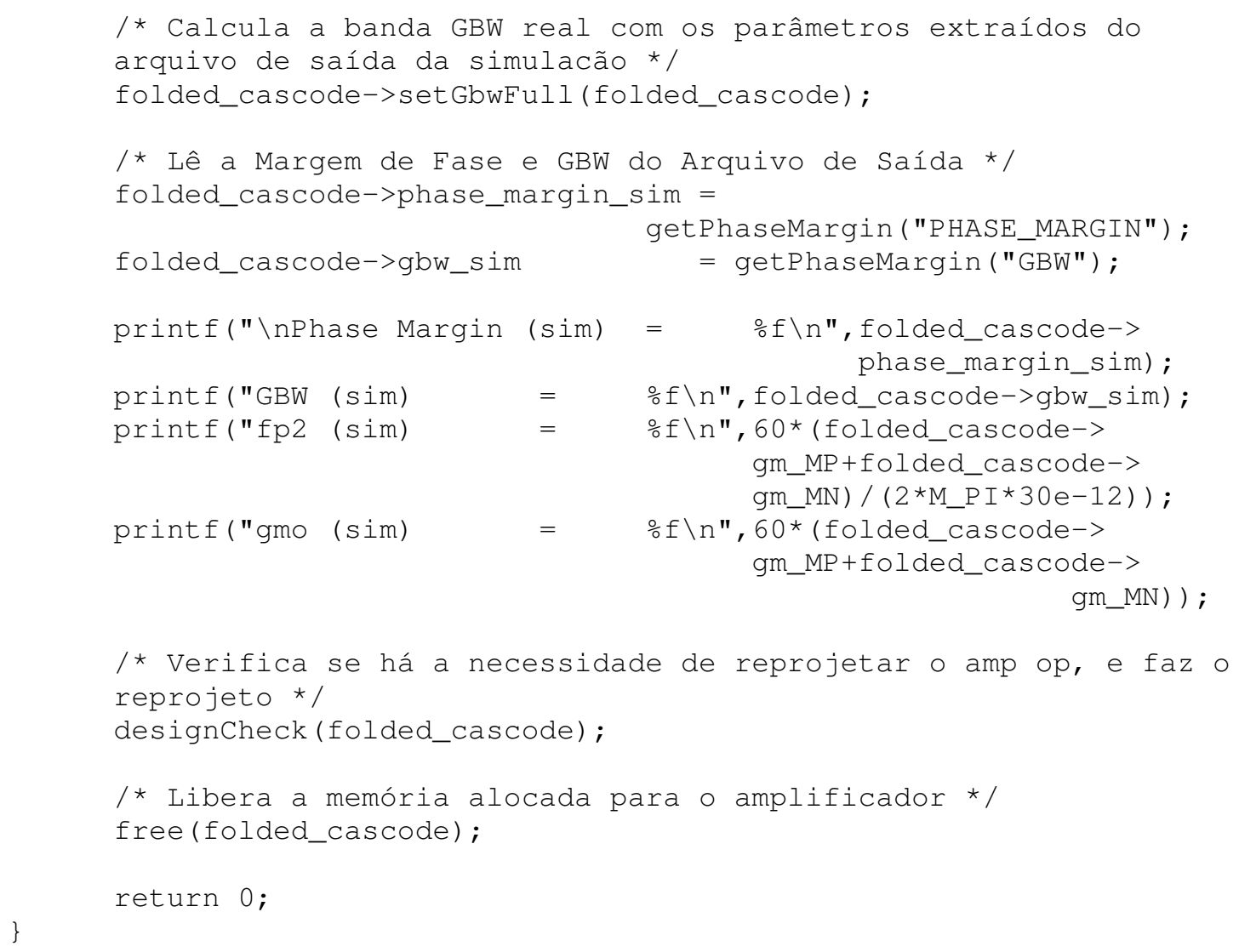

